Supporting Information for

\title{
Cu-catalyzed Transformation of Alkynes and Alkenes with Azide and Dimethyl Sulfoxide Reagents
}

Tao Shen, ${ }^{1}$ Xiaoqiang Huang, ${ }^{1}$ Yu-Feng Liang ${ }^{1}$ and Ning Jiao ${ }^{*}, 1,2$

\footnotetext{
${ }^{1}$ State Key Laboratory of Natural and Biomimetic Drugs, School of Pharmaceutical Sciences, Peking University, Xue Yuan Rd. 38, Beijing 100083, China; ${ }^{2}$ State Key Laboratory of Organometallic Chemistry, Chinese Academy of Sciences, Shanghai 200032, China.

E-mail: jiaoning@pku.edu.cn; Fax: (+86)10-82805297
}

General Remarks

Additional Screening with Different Reaction Conditions

Mechanistic Studies

Experiments and characterization of products

NMR Spectra

X-ray structure of $\mathbf{2 e}$

S109 


\section{General Remarks}

All commercially available compounds were purchased from Sigma-Aldrich, Adamas, Alfa-Aesar and Acros. Solvents were from Beijing Ouhe and Beijing Chemical Works and used as received without further purification. Reactions were performed without regard for exclusion of ambient air or moisture in a schlenk tube under standard conditions (specific reaction conditions are described below). Analysis of crude reaction mixture was done on an Agilent 7890 GC System with an Agilent 5975 Mass Selective Detector. Products were purified by flash chromatography or by preparative thin-layer chromatography on silica gel. ${ }^{1}$ Chemical shifts (in ppm) were referenced to tetramethylsilane $(\delta=0 \mathrm{ppm})$ in $\mathrm{CDCl}_{3}$ or dimethyl sulfoxide $(\delta=2.49 \mathrm{ppm})$ in DMSO-d $\mathrm{d}_{6}$ as an internal standard. ${ }^{13} \mathrm{C}$-NMR spectra were obtained by the same NMR spectrometers and were calibrated with $\mathrm{CDCl}_{3}(\delta=77.00 \mathrm{ppm})$ or DMSO-d $\mathrm{d}_{6}(\delta=39.50 \mathrm{ppm})$. Mass spectra were recorded using a PE SCLEX QSTAR spectrometer. High resolution mass spectra were obtained with a Bruker APEX IV Fourier transform ion cyclotron resonance mass spectrometer using electrospray ionisation (ESI). Fourier-transform infrared (FTIR) spectra were obtained with a Nicolet Nexus 470 Fourier transform infrared spectrometer. 
Table S1. Screening of the Reaction Conditions ${ }^{a}$

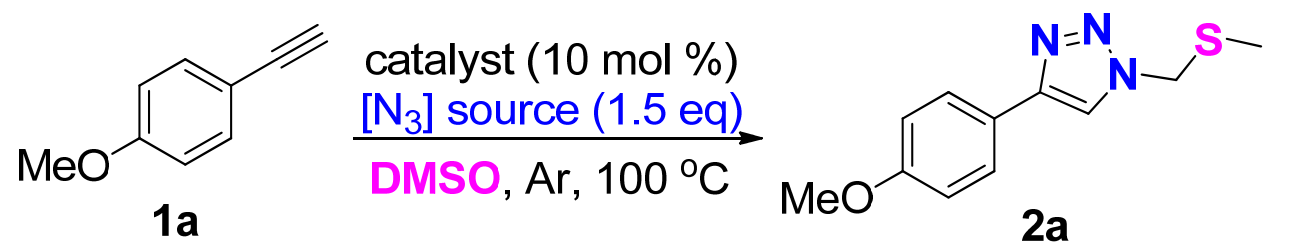

\begin{tabular}{|c|c|c|c|}
\hline entry & catalyst & {$\left[\mathrm{N}_{3}\right]$ source } & yield of $2 a^{b}(\%)$ \\
\hline 1 & $\mathrm{Ag}_{2} \mathrm{CO}_{3}$ & $\mathrm{TMSN}_{3}$ & 8 \\
\hline 2 & $\mathrm{AuCl}_{3}$ & $\mathrm{TMSN}_{3}$ & 0 \\
\hline 3 & $\mathrm{NiCl}_{2}$ & $\mathrm{TMSN}_{3}$ & 0 \\
\hline 4 & $\mathrm{Cu}(\mathrm{acac})_{2}$ & $\mathrm{TMSN}_{3}$ & 45 \\
\hline 5 & $\mathrm{Cu}(\mathrm{acac})_{2}$ & DPPA & $\begin{array}{l}45 \\
86\end{array}$ \\
\hline 6 & $\mathrm{Cu}(\mathrm{acac})_{2}$ & $\mathrm{NaN}_{3}$ & 0 \\
\hline 7 & $\mathrm{Cu}(\mathrm{acac})_{2}$ & $\mathrm{TsN}_{3}$ & 0 \\
\hline 8 & $\mathrm{Cu}(\mathrm{OAc})_{2}$ & DPPA & 61 \\
\hline 9 & CuBr & DPPA & 52 \\
\hline $10^{c}$ & $\mathrm{Cu}(\mathrm{acac})_{2}$ & DPPA & 0 \\
\hline 11 & & DPPA & 0 \\
\hline $12^{d}$ & $\mathrm{Cu}(\text { acac })_{2}$ & DPPA & 44 \\
\hline $13^{e}$ & $\mathrm{Cu}(\mathrm{acac})_{2}$ & DPPA & 88 \\
\hline $14^{f}$ & $\mathrm{Cu}(\mathrm{acac})_{2}$ & DPPA & 31 \\
\hline 15 & $\mathrm{CuCl}_{2}$ & DPPA & 38 \\
\hline 16 & $\mathrm{CuCl}$ & DPPA & 41 \\
\hline 17 & Cul & DPPA & 63 \\
\hline 18 & $\mathrm{Cu}(\mathrm{OTf})_{2}$ & DPPA & 68 \\
\hline 19 & $\mathrm{CuCN}$ & DPPA & 63 \\
\hline 20 & $\mathrm{CuO}$ & DPPA & 60 \\
\hline
\end{tabular}

${ }^{a}$ Reaction conditions: 1a $(0.5 \mathrm{mmol})$, catalyst $(0.05 \mathrm{mmol})$, azide source $(0.75 \mathrm{mmol})$, dry DMSO $(2 \mathrm{~mL})$, stirred at $100{ }^{\circ} \mathrm{C}$ under $\mathrm{Ar}$ for $12 \mathrm{~h}$, this reaction is sensitive to water. ${ }^{b}$ Isolated yields. ${ }^{c} \mathrm{At}$ $50{ }^{\circ} \mathrm{C}$. ${ }^{d}$ At $80{ }^{\circ} \mathrm{C} .{ }^{e} 2.0$ Equiv of DPPA was used. ${ }^{f}$ The reaction was carried out under $\mathrm{O}_{2}$. DPPA $=$ diphenylphosphoryl azide, $\mathrm{TMS}=$ trimethylsilyl, $\mathrm{DMSO}=$ dimethylsulfoxide, $\mathrm{Ts}=$ p-toluenesulfonyl.

Table S2. Reaction results with different stoichiometric amount of DMSO.

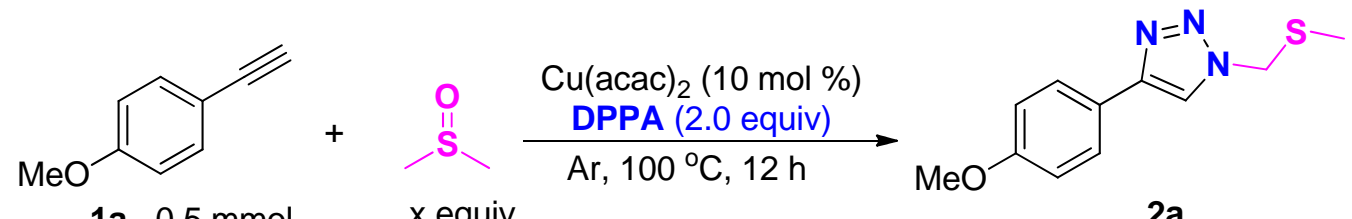
1a $0.5 \mathrm{mmol}$ $x$ equiv
$2 a$

\begin{tabular}{cl}
\hline $\mathrm{x}$ equiv & yield \\
\hline 2.0 & trace \\
4.0 & trace \\
6.0 & $9 \%$ \\
8.0 & $21 \%$ \\
10.0 & $33 \%$ \\
\hline
\end{tabular}


Reaction results with different other kinds of alkenes.

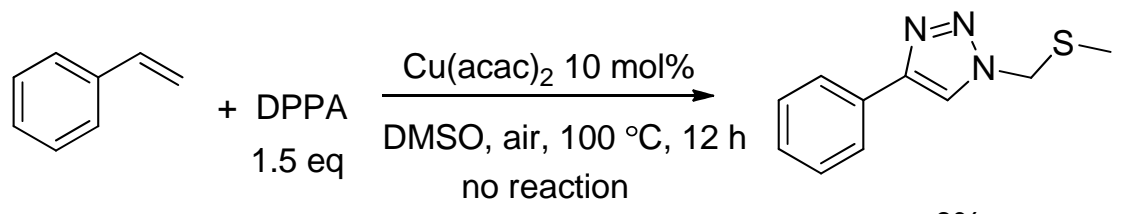

$0 \%$

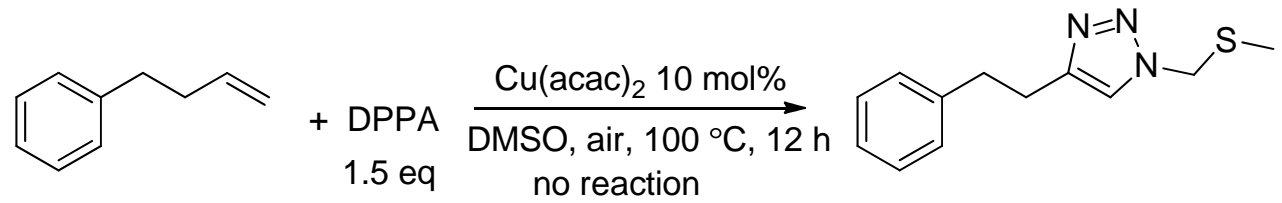

$0 \%$

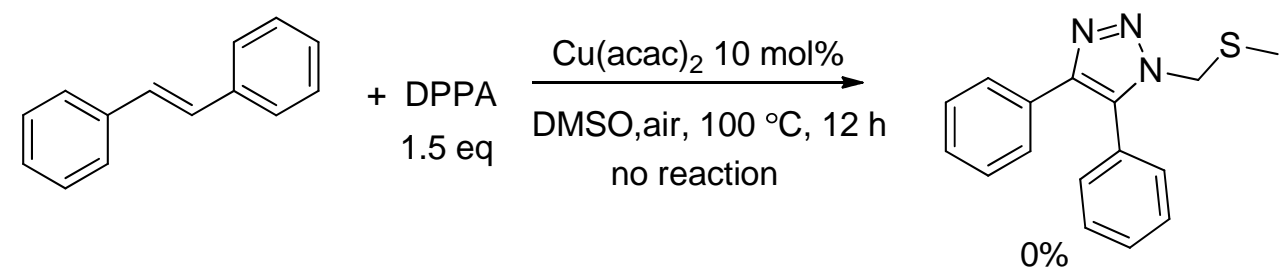

Reaction result of 3a under Ar.

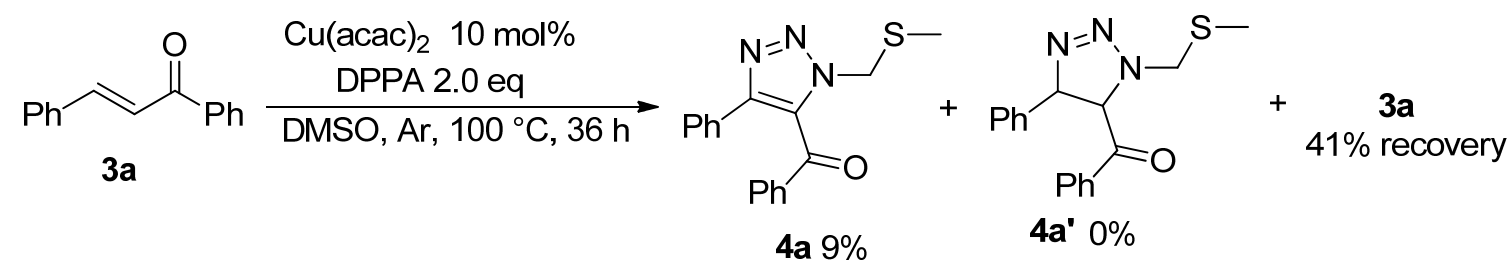

\section{Mechanistic Studies:}<smiles>C#Cc1ccc(OC)cc1</smiles>

1a

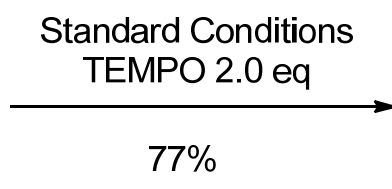

$77 \%$

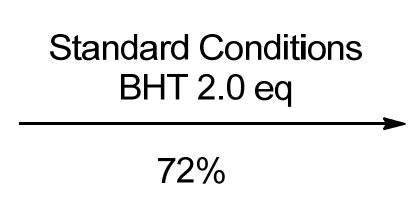<smiles>COc1ccc(-c2cn(CSC)nn2)cc1</smiles>

2a
Eq. 1<smiles>COc1ccc(-c2cn(CSC)nn2)cc1</smiles>

$2 a$
Eq. 2

Eq1: The reaction of $1 \mathrm{a}(66.1 \mathrm{mg}, 0.5 \mathrm{mmol}), \mathrm{DPPA}(206.2 \mathrm{mg}, 0.75 \mathrm{mmol}), \mathrm{Cu}(\mathrm{acac})_{2}(13.0 \mathrm{mg}$, $0.05 \mathrm{mmol})$, TEMPO (156 mg, $1.0 \mathrm{mmol})$ in dry DMSO $(2 \mathrm{~mL})$ afforded $90.5 \mathrm{mg}(77 \%)$ of $2 \mathbf{a}$.

Eq2: The reaction of 1a $(66.0 \mathrm{mg}, 0.5 \mathrm{mmol})$, DPPA (206.2 $\mathrm{mg}, 0.75 \mathrm{mmol}), \mathrm{Cu}(\mathrm{acac})_{2}(13.0 \mathrm{mg}$, $0.05 \mathrm{mmol})$, BHT $(220 \mathrm{mg}, 1.0 \mathrm{mmol})$ in dry DMSO $(2 \mathrm{~mL})$ afforded $84.6 \mathrm{mg}(72 \%)$ of $2 \mathbf{a}$. 


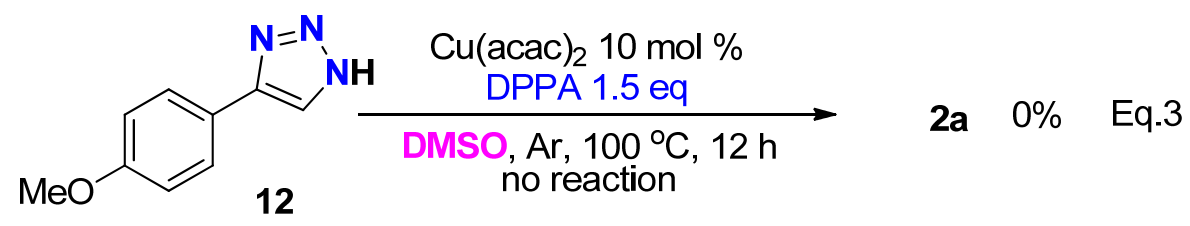

The triazoles 12 was synthesized by traditional click reaction from $1 \mathrm{a}$ and $\mathrm{NaN}_{3}$.

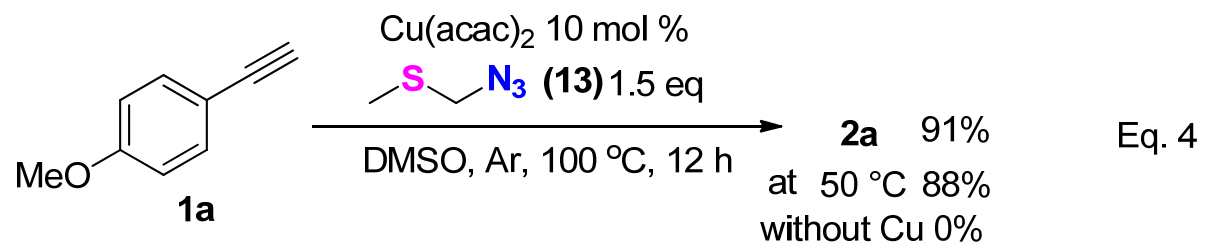

The (azidomethyl)(methyl)sulfane was synthesized as following method: The solution of $\mathrm{SOCl}_{2}$ ( $2.90 \mathrm{~g}, 22 \mathrm{mmol})$ in $20 \mathrm{~mL}$ DCM was dropwised to a solution of DMSO (1.56 g, $20 \mathrm{mmol})$ in 20 $\mathrm{mL}$ DCM at room temperature, heated for $4 \mathrm{~h}$, the solvent was evaporated under vaccum, a colorless oil (1.35 g, 66\%) was obtained by vacuum distillation. (Azidomethyl)(methyl)sulfane 13:

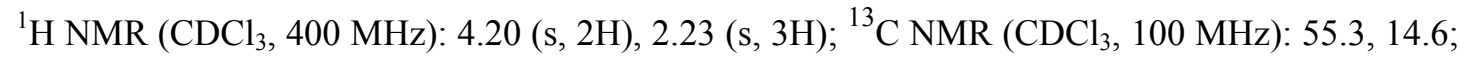
MS (70 eV): m/z (\%): $103.0(25)$.

Eq4: To a $25 \mathrm{~mL}$ Schlenk tube was added $\mathrm{Cu}(\mathrm{acac})_{2}(13.0 \mathrm{mg}, 10 \mathrm{~mol} \%)$, and the tube was purged with $\mathrm{Ar}$ for three times, followed by addition of alkyne 1a $(66.0 \mathrm{mg}, 0.5 \mathrm{mmol})$, (azidomethyl)(methyl)sulfane $(77.2 \mathrm{mg}, 0.75 \mathrm{mmol})$ and dry DMSO $(2.0 \mathrm{~mL})$. The formed mixture was gradually heated at $100{ }^{\circ} \mathrm{C}$ under $\operatorname{Ar}(1 \mathrm{~atm})$ and stirred for $12 \mathrm{~h}$ as monitored by TLC. The solution was then cooled to room temperature, diluted with ethyl acetate $(30 \mathrm{~mL})$, washed with $\mathrm{H}_{2} \mathrm{O}(3 \times 10 \mathrm{~mL})$, dried over $\mathrm{MgSO}_{4}$, filtered, and evaporated under vaccum. The crude product was purified by column chromatography on silica gel (eluent: petroleum ether / ethyl acetate $=5: 1)$ to afford procuct $2 \mathbf{a}(107.0 \mathrm{mg}, 91 \%)$. The reaction carried out under $50{ }^{\circ} \mathrm{C}$ gived 2a (103.4 mg, 88\%).

\section{The reaction of DMSO and DPPA}

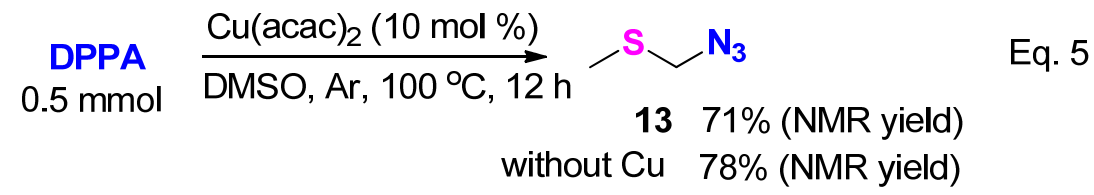

Eq.5: The reaction of DMSO $(2.0 \mathrm{~mL})$, DPPA $(165.0 \mathrm{mg}, 0.5 \mathrm{mmol}), \mathrm{Cu}(\mathrm{acac})_{2}(13.0 \mathrm{mg}, 0.05$ mmol) at $100{ }^{\circ} \mathrm{C}$ under $\mathrm{Ar}$ for $12 \mathrm{~h}$, afforded 71\% NMR yield of 13. The reaction of DMSO (2.0 $\mathrm{mL}$ ), DPPA (165.0 mg, $0.5 \mathrm{mmol})$, at $100{ }^{\circ} \mathrm{C}$ under Ar for $12 \mathrm{~h}$, afforded $78 \%$ NMR yield of 13 . 


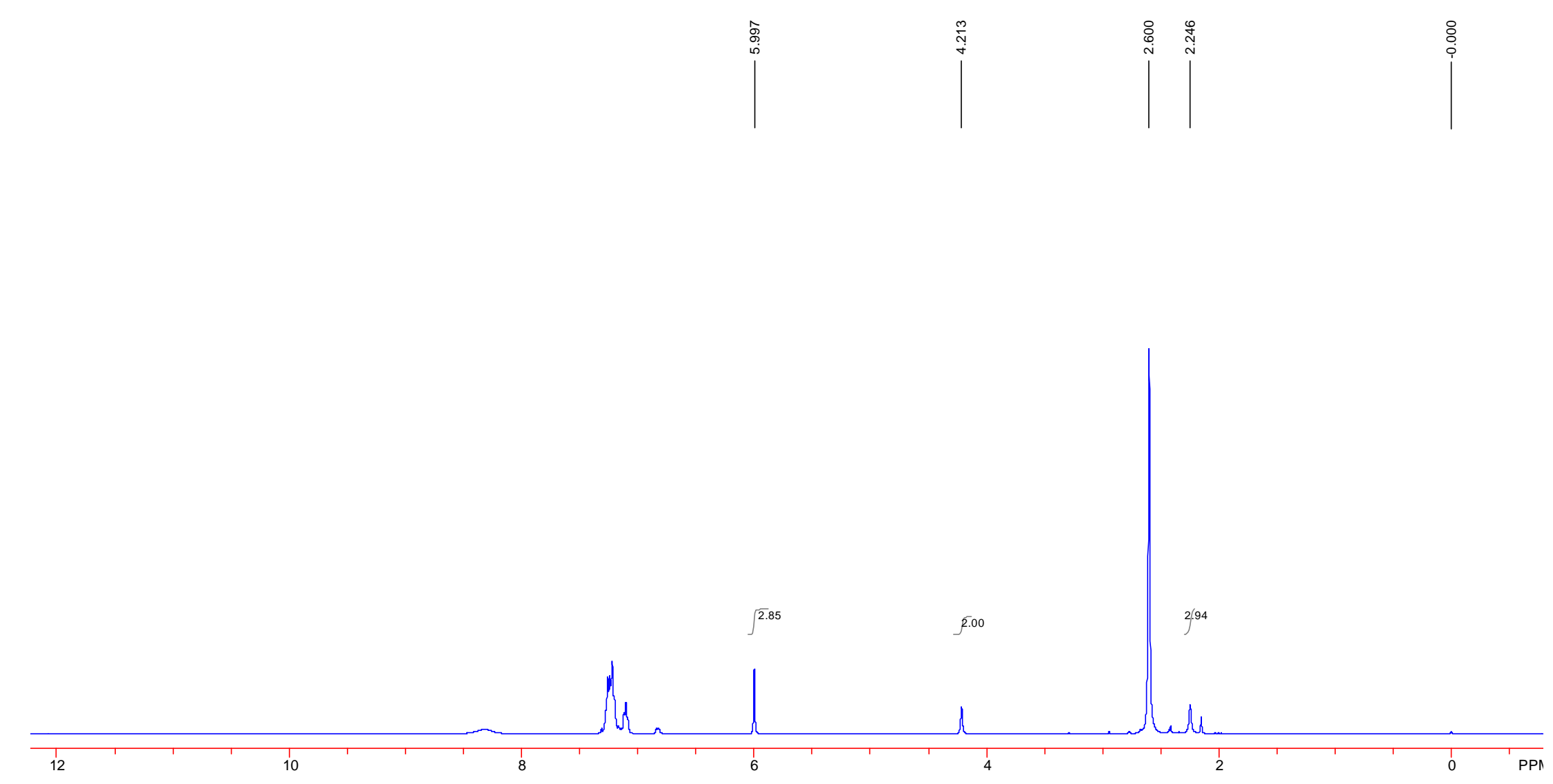

${ }^{1} \mathrm{H} \mathrm{NMR}\left(\mathrm{CDCl}_{3}, 400 \mathrm{MHz}\right)$ of reaction mixture with $\mathrm{Cu}(\mathrm{acac})_{2}$ (using $0.5 \mathrm{mmol}$ 1,1-2,2-tetrachloroethane as internal standard) 


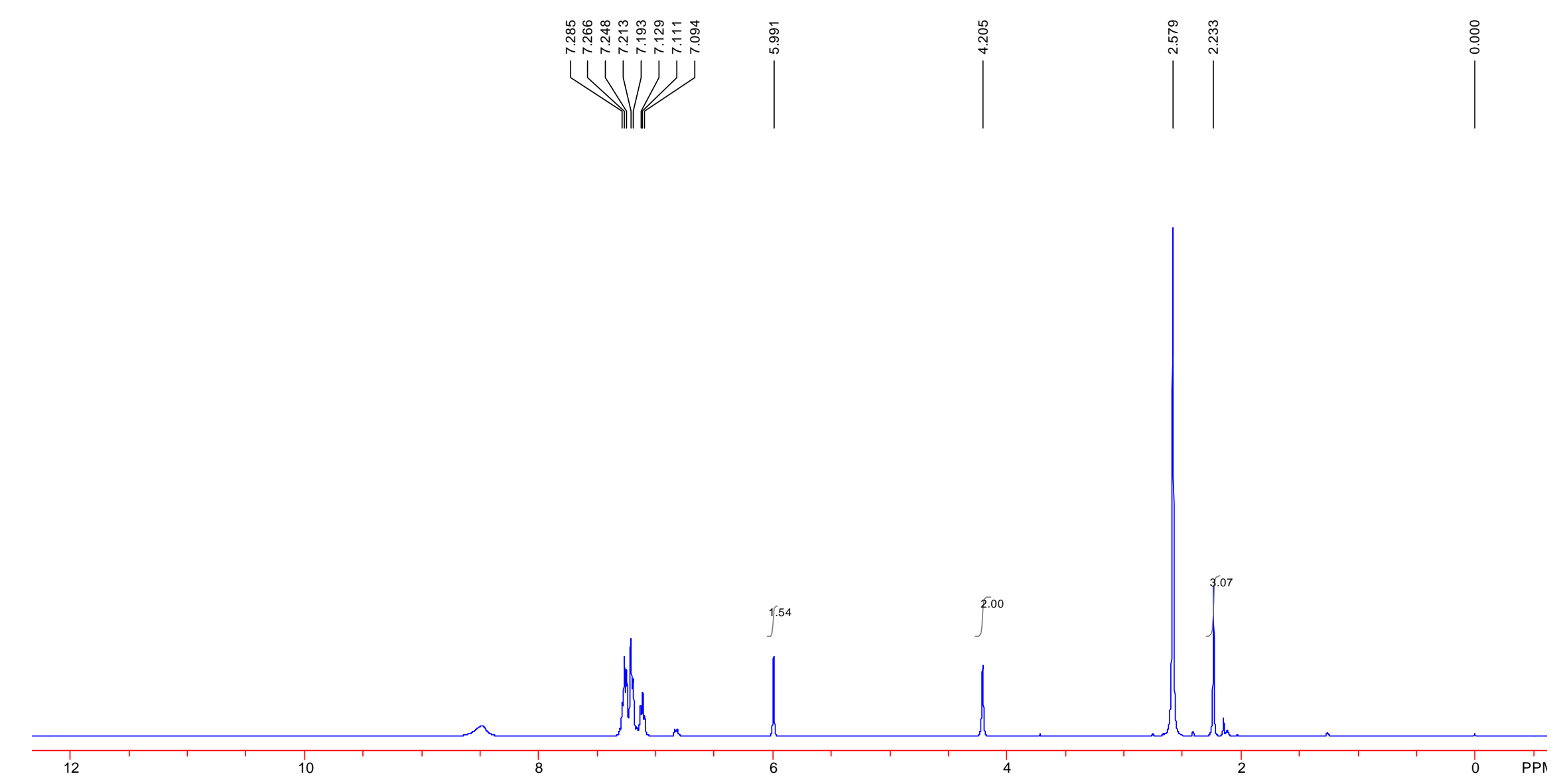

${ }^{1} \mathrm{H} \mathrm{NMR}\left(\mathrm{CDCl}_{3}, 400 \mathrm{MHz}\right)$ of reaction mixture without $\mathrm{Cu}(\mathrm{acac})_{2}$ (using $0.3 \mathrm{mmol}$ 1,1-2,2-tetrachloroethane as internal standard) 


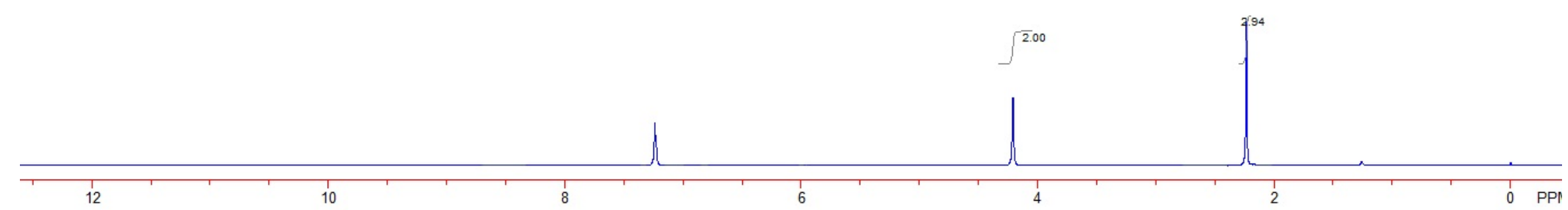

${ }^{1} \mathrm{H} \mathrm{NMR}\left(\mathrm{CDCl}_{3}, 400 \mathrm{MHz}\right)$ of (azidomethyl)(methyl)sulfane 13 


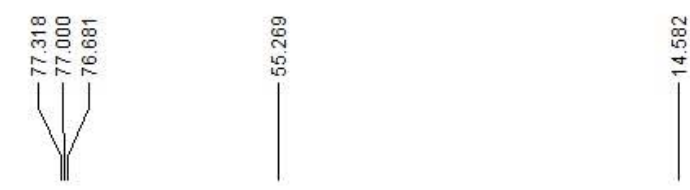

S. $\mathrm{N}_{3}$

13

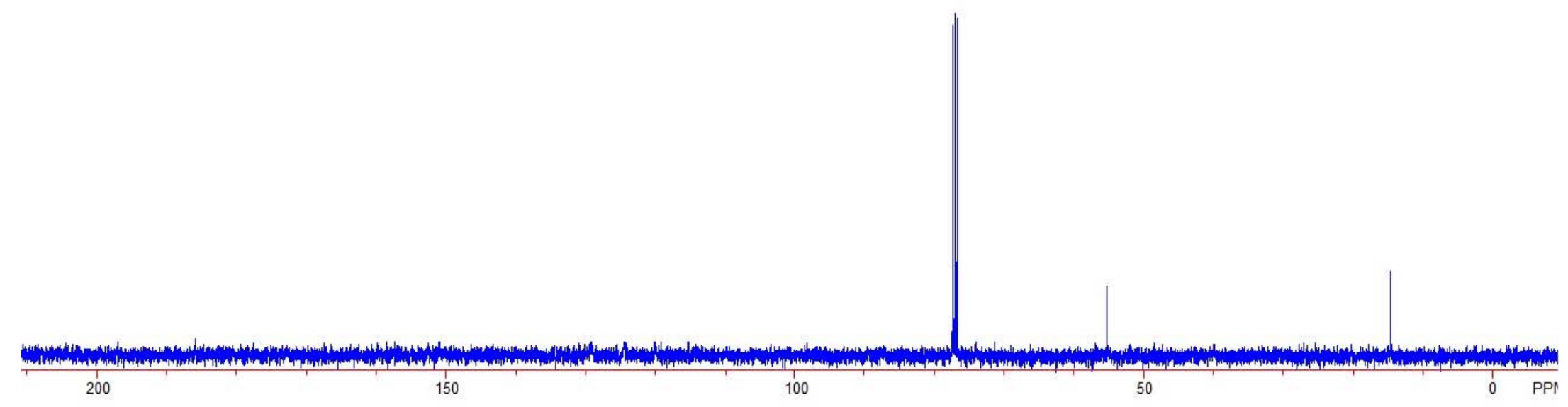

${ }^{13} \mathrm{C}$ NMR ( $\mathrm{CDCl}_{3}, 400 \mathrm{MHz}$ ) of (azidomethyl)(methyl)sulfane 13 


\section{Experimental section}

Typical procedure: To a $25 \mathrm{~mL}$ Schlenk tube was added $\mathrm{Cu}(\mathrm{acac})_{2}(13.0 \mathrm{mg}, 10 \mathrm{~mol} \%)$, and the tube was purged with Ar for three times, followed by addition of alkynes $1(0.5 \mathrm{mmol})$, DPPA (206 mg, $0.75 \mathrm{mmol})$ and dry DMSO $(2.0 \mathrm{~mL}$ ). The formed mixture was gradually heated at 100 ${ }^{\circ} \mathrm{C}$ under $\operatorname{Ar}(1 \mathrm{~atm})$ and stirred for $12 \mathrm{~h}$ as monitored by TLC. The solution was then cooled to rt, diluted with ethyl acetate $(30 \mathrm{~mL})$, washed with $\mathrm{H}_{2} \mathrm{O}(3 \times 10 \mathrm{~mL})$, dried over $\mathrm{MgSO}_{4}$, filtered, and evaporated under vaccum. The crude product was purified by column chromatography on silica gel (eluent: petroleum ether / ethyl acetate $=5: 1$ ) to afford procucts 2 .

Characterization of new compounds.<smiles>COc1ccc(-c2cn(CSC)nn2)cc1</smiles>

$2 a$

\section{1) 4-(4-Methoxyphenyl)-1-((methylthio)methyl)-1H-1,2,3-triazole (2a)}

The reaction of 1a $(66.1 \mathrm{mg}, 0.5 \mathrm{mmol})$, DPPA (206.2 mg, $0.75 \mathrm{mmol}), \mathrm{Cu}(\mathrm{acac})_{2}(13.0 \mathrm{mg}, 0.05$ mmol) in dry DMSO $(2 \mathrm{~mL})$ afforded $101.0 \mathrm{mg}(86 \%)$ of $2 \mathbf{2 a}$; $2 \mathbf{a}$ : yellow solid; ${ }^{1} \mathrm{H}$ NMR (d6-DMSO, $400 \mathrm{MHz}): 8.53$ (s, 1H), $7.81(\mathrm{~d}, J=8.8 \mathrm{~Hz}, 2 \mathrm{H}), 7.01(\mathrm{~d}, J=8.8 \mathrm{~Hz}, 2 \mathrm{H}), 5.54$ (s, $2 \mathrm{H}), 3.78$ (s, 3H), 2.18 (s, 3H); ${ }^{13} \mathrm{C}$ NMR (d ${ }_{6}$-DMSO, $\left.100 \mathrm{MHz}\right): 159.6,147.3,127.1,123.6$, 120.5, 114.8, 55.6, 52.3, 15.1; HRMS: calc. for $\mathrm{C}_{11} \mathrm{H}_{14} \mathrm{~N}_{3} \mathrm{OS}(\mathrm{M}+\mathrm{H})^{+}$, 236.0852, found, 236.0852 .<smiles>CCOc1ccc(-c2cn(CSC)nn2)cc1</smiles>

\section{$2 b$}

\section{2) 4-(4-Ethoxyphenyl)-1-((methylthio)methyl)-1H-1,2,3-triazole (2b)}

The reaction of $\mathbf{1 b}(73.0 \mathrm{mg}, 0.5 \mathrm{mmol})$, DPPA (206.2 mg, $0.75 \mathrm{mmol}), \mathrm{Cu}(\mathrm{acac})_{2}(13 \mathrm{mg}, 0.05$ mmol) in dry DMSO (2 mL) afforded $88.4 \mathrm{mg}(71 \%)$ of $\mathbf{2 b}$; $2 \mathbf{b}$ : yellow solid; ${ }^{1} \mathrm{H}$ NMR (d $\mathrm{d}_{6}$-DMSO, $\left.400 \mathrm{MHz}\right): 8.53$ (s, 1H), $7.78(\mathrm{~d}, J=7.2 \mathrm{~Hz}, 2 \mathrm{H}), 6.99$ (d, $\left.J=7.2 \mathrm{~Hz}, 2 \mathrm{H}\right), 5.54$ (s, 2H), 4.04 (q, $J=6.8 \mathrm{~Hz}, 2 \mathrm{H}), 2.18(\mathrm{~s}, 3 \mathrm{H}), 1.34$ (t, $J=6.8 \mathrm{~Hz}, 3 \mathrm{H}) ;{ }^{13} \mathrm{C}$ NMR (d $\mathrm{d}_{6}$-DMSO, 100 MHz): 158.8, 147.3, 127.0, 123.5, 120.4, 115.2, 63.5, 52.3, $15.1\left(\mathbf{S C H}_{3}\right.$ and $\left.\mathrm{OCH}_{2} \mathbf{C H}_{3}\right)$; HRMS: calc. for $\mathrm{C}_{12} \mathrm{H}_{16} \mathrm{~N}_{3} \mathrm{OS}(\mathrm{M}+\mathrm{H})^{+}$, 250.1009; found, 250.1010 .<smiles>CSCn1cc(-c2ccc(F)cc2)nn1</smiles>

\section{2c}

\section{3) 4-(4-Fluorophenyl)-1-((methylthio)methyl)-1H-1,2,3-triazole (2c)}

The reaction of 1c $(60.6 \mathrm{mg}, 0.5 \mathrm{mmol})$, DPPA $(206.2 \mathrm{mg}, 0.75 \mathrm{mmol}), \mathrm{Cu}(\mathrm{acac})_{2}(13 \mathrm{mg}, 0.05$ $\mathrm{mmol})$ in dry DMSO $(2 \mathrm{~mL})$ afforded $74.7 \mathrm{mg}(67 \%)$ of 2 c; 2 c: yellow solid; ${ }^{1} \mathrm{H}$ NMR $\left(\mathrm{d}_{6}\right.$-DMSO, 
$400 \mathrm{MHz}): 8.63(\mathrm{~s}, 1 \mathrm{H}), 7.91(\mathrm{dd}, J=7.6 \mathrm{~Hz}, J=5.6 \mathrm{~Hz}, 2 \mathrm{H}), 7.28(\mathrm{t}, J=8.8 \mathrm{~Hz}, 2 \mathrm{H}), 5.56(\mathrm{~s}$, 2H), 2.18 (s, 3H); ${ }^{13} \mathrm{C}$ NMR (d $\mathrm{d}_{6}$-DMSO, $\left.100 \mathrm{MHz}\right): 162.4(\mathrm{~d}, J=243.3 \mathrm{~Hz}), 146.5,127.8(\mathrm{~d}, J$ $=8.2 \mathrm{~Hz}), 127.6(\mathrm{~d}, J=2.6 \mathrm{~Hz}), 121.4,116.3(\mathrm{~d}, J=21.8 \mathrm{~Hz}), 52.5,15.1$; HRMS: calc. for $\mathrm{C}_{10} \mathrm{H}_{11} \mathrm{FN}_{3} \mathrm{~S}(\mathrm{M}+\mathrm{H})^{+}, 224.0652$, found, 224.0653<smiles>CSCn1cc(-c2ccc(Cl)cc2)nn1</smiles>

2d

4) 4-(4-Chlorophenyl)-1-((methylthio)methyl)-1H-1,2,3-triazole (2d)

The reaction of $1 \mathbf{d}(68.1 \mathrm{mg}, 0.5 \mathrm{mmol})$, DPPA (206.2 mg, $0.75 \mathrm{mmol}), \mathrm{Cu}(\mathrm{acac})_{2}(13 \mathrm{mg}, 0.05$ mmol) in dry DMSO $(2 \mathrm{~mL})$ afforded $71.7 \mathrm{mg}(60 \%)$ of $\mathbf{2 d}$; $2 \mathbf{2 d}$ : white solid; ${ }^{1} \mathrm{H}$ NMR ( $\mathrm{d}_{6}$-DMSO, $400 \mathrm{MHz}): 8.70$ (s, 1H), 7.90 (d, $J=8.8 \mathrm{~Hz}, 2 \mathrm{H}), 7.50$ (d, $J=8.8 \mathrm{~Hz}, 2 \mathrm{H}), 5.57$ (s, 2H), 2.18 (s, $3 \mathrm{H}) ;{ }^{13} \mathrm{C}$ NMR (d $\mathrm{d}_{6}$-DMSO, $\left.100 \mathrm{MHz}\right): 146.2,132.9,129.9,129.4,127.4,121.8,52.5,15.1$; HRMS: calc. for $\mathrm{C}_{10} \mathrm{H}_{11} \mathrm{ClN}_{3} \mathrm{~S}(\mathrm{M}+\mathrm{H})^{+}, 240.0357$, found, 240.0363 .<smiles>CSCn1cc(-c2ccc(Br)cc2)nn1</smiles>

$2 \mathrm{e}$

5) 4-(4-Bromophenyl)-1-((methylthio)methyl)-1H-1,2,3-triazole (2e)

The reaction of 1e ( $90.3 \mathrm{mg}, 0.5 \mathrm{mmol})$, DPPA (206.2 mg, $0.75 \mathrm{mmol}), \mathrm{Cu}(\mathrm{acac})_{2}(13 \mathrm{mg}, 0.05$ mmol) in dry DMSO $(2 \mathrm{~mL})$ afforded $104.3 \mathrm{mg}(74 \%)$ of $\mathbf{2 e}$; 2e: yellow solid; ${ }^{1} \mathrm{H}$ NMR ( $\mathrm{d}_{6}$-DMSO, $\left.400 \mathrm{MHz}\right): 8.70(\mathrm{~s}, 1 \mathrm{H}), 7.84(\mathrm{~d}, J=8.4 \mathrm{~Hz}, 2 \mathrm{H}), 7.64(\mathrm{~d}, J=8.4 \mathrm{~Hz}, 2 \mathrm{H}), 5.57(\mathrm{~s}$, 2H), 2.18 (s, 3H); ${ }^{13} \mathrm{C}$ NMR (d ${ }_{6}$-DMSO, $100 \mathrm{MHz}$ ): 146.2, 132.3, 130.3, 127.7, 121.9, 121.4, 52.4, 15.1; HRMS: calc. for $\mathrm{C}_{10} \mathrm{H}_{11} \mathrm{BrN}_{3} \mathrm{~S}(\mathrm{M}+\mathrm{H})^{+}$, 283.9852, found, 283.9855 .<smiles>CSCn1cc(-c2ccc(C(F)(F)F)cc2)nn1</smiles>

\section{6) 1-((Methylthio)methyl)-4-(4-(trifluoromethyl)phenyl)-1H-1,2,3-triazole (2f)}

The reaction of 1 f $(85.4 \mathrm{mg}, 0.5 \mathrm{mmol})$, DPPA $(206.2 \mathrm{mg}, 0.75 \mathrm{mmol}), \mathrm{Cu}(\mathrm{acac})_{2}(13 \mathrm{mg}, 0.05$ $\mathrm{mmol})$ in dry DMSO (2 mL) afforded $91.4 \mathrm{mg}(67 \%)$ of $\mathbf{2 f}$; 2f: white solid; ${ }^{1} \mathrm{H}$ NMR $\left(\mathrm{d}_{6}\right.$-DMSO, $400 \mathrm{MHz}$ ): $8.82(\mathrm{~s}, 1 \mathrm{H}), 8.10$ (d, $J=8.0 \mathrm{~Hz}, 2 \mathrm{H}), 7.79$ (d, $J=8.0 \mathrm{~Hz}, 2 \mathrm{H}), 5.60$ (s, 2H), 2.19 (s, $3 \mathrm{H}) ;{ }^{13} \mathrm{C}$ NMR (d $\mathrm{d}_{6}$-DMSO, $\left.100 \mathrm{MHz}\right): 146.0,135.0,127.8$ (q, $\left.J=274.2 \mathrm{~Hz}\right), 126.4,126.34$, 126.30, 122.8, 52.6, 15.1; HRMS: calc. for $\mathrm{C}_{11} \mathrm{H}_{11} \mathrm{~F}_{3} \mathrm{~N}_{3} \mathrm{~S}(\mathrm{M}+\mathrm{H})^{+}$, 274.0620, found, 274.0624. 
<smiles>CSCn1cc(-c2ccc(C#N)cc2)nn1</smiles>

$2 g$

\section{7) 4-(1-((Methylthio)methyl)-1H-1,2,3-triazol-4-yl)benzonitrile (2g)}

The reaction of $1 \mathrm{~g}(64.0 \mathrm{mg}, 0.5 \mathrm{mmol})$, DPPA (206.2 $\mathrm{mg}, 0.75 \mathrm{mmol}), \mathrm{Cu}(\mathrm{acac})_{2}(13 \mathrm{mg}, 0.05$ $\mathrm{mmol})$ in dry DMSO $(2 \mathrm{~mL})$ afforded $94.3 \mathrm{mg}(82 \%)$ of $\mathbf{2 g}$; $2 \mathbf{g}$ : yellow solid; ${ }^{1} \mathrm{H}$ NMR $\left(\mathrm{d}_{6}-\mathrm{DMSO}\right.$, $400 \mathrm{MHz}$ ): $8.86(\mathrm{~s}, 1 \mathrm{H}), 8.08$ (dd, $J=6.4 \mathrm{~Hz}, J=1.6 \mathrm{~Hz}, 2 \mathrm{H}), 7.91(\mathrm{dd}, J=6.4 \mathrm{~Hz}, J=1.6 \mathrm{~Hz}$, $2 \mathrm{H}), 5.60(\mathrm{~s}, 2 \mathrm{H}), 2.19$ (s, 3H); ${ }^{13} \mathrm{C}$ NMR (d 6 -DMSO, $\left.100 \mathrm{MHz}\right): 145.7,135.4,133.4,126.3$, 123.2, 119.2, 110.7, 52.6, 15.1; HRMS: calc. for $\mathrm{C}_{11} \mathrm{H}_{11} \mathrm{~N}_{4} \mathrm{~S}(\mathrm{M}+\mathrm{H})^{+}, 231.0699$, found, 231.0699.<smiles>CSCn1cc(-c2ccc(C)cc2)nn1</smiles>

$2 h$

\section{8) 1-((Methylthio)methyl)-4-(p-tolyl)-1H-1,2,3-triazole (2h)}

The reaction of $\mathbf{1 h}(66.2 \mathrm{mg}, 0.5 \mathrm{mmol})$, DPPA (206.2 mg, $0.75 \mathrm{mmol}), \mathrm{Cu}(\mathrm{acac})_{2}(13 \mathrm{mg}, 0.05$ mmol) in dry DMSO $(2 \mathrm{~mL})$ afforded $69.0 \mathrm{mg}(63 \%)$ of $\mathbf{2 h}$; $2 \mathbf{h}$ : yellow solid; ${ }^{1} \mathrm{H}$ NMR (d $\mathrm{d}_{6}$-DMSO, $\left.400 \mathrm{MHz}\right): 8.59$ (s, 1H), $7.77(\mathrm{~d}, J=8.0 \mathrm{~Hz}, 2 \mathrm{H}), 7.25$ (d, $\left.J=8.0 \mathrm{~Hz}, 2 \mathrm{H}\right), 5.55$ (s, 2H), 2.32 (s, 3H), 2.18 (s, 3H); ${ }^{13} \mathrm{C}$ NMR (d 6 -DMSO, $\left.100 \mathrm{MHz}\right): 147.4,137.8,129.9,128.3$, 125.7, 121.0, 52.4, 21.3, 15.1; HRMS: calc. for $\mathrm{C}_{11} \mathrm{H}_{14} \mathrm{~N}_{3} \mathrm{~S}(\mathrm{M}+\mathrm{H})^{+}, 220.0903$, found, 220.0905 .

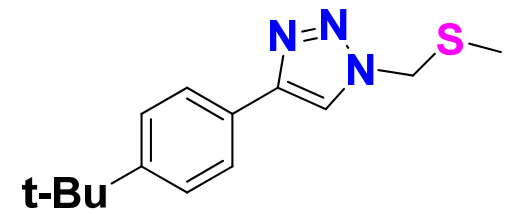

2i

9) 4-(4-(tert-Butyl)phenyl)-1-((methylthio)methyl)-1H-1,2,3-triazole (2i)

The reaction of $1 \mathbf{i}(79.0 \mathrm{mg}, 0.5 \mathrm{mmol})$, DPPA $(206.2 \mathrm{mg}, 0.75 \mathrm{mmol}), \mathrm{Cu}(\mathrm{acac})_{2}(13 \mathrm{mg}, 0.05$ mmol) in dry DMSO $(2 \mathrm{~mL})$ afforded $104.4 \mathrm{mg}(80 \%)$ of $2 \mathbf{i}$; $2 \mathbf{i}$ : yellow solid; ${ }^{1} \mathrm{H}$ NMR (d 6 -DMSO, $400 \mathrm{MHz}): 8.59$ (s, 1H), 7.80 (d, $J=8.4 \mathrm{~Hz}, 2 \mathrm{H}), 7.46$ (d, $J=8.4 \mathrm{~Hz}, 2 \mathrm{H}), 5.56$ (s, 2H), 2.18 (s, $3 \mathrm{H}), 1.29$ (s, 9H); ${ }^{13} \mathrm{C}$ NMR (d ${ }_{6}$-DMSO, $\left.100 \mathrm{MHz}\right): 150.9,147.3,128.2,126.0,125.5,121.1,52.3$, 34.7, 31.4, 15.1; HRMS: calc. for $\mathrm{C}_{14} \mathrm{H}_{20} \mathrm{~N}_{3} \mathrm{~S}(\mathrm{M}+\mathrm{H})^{+}, 262.1372$, found, 262.1377 .<smiles>CSCn1cc(-c2ccc(-n3cccc3)cc2)nn1</smiles>

10) 4-(4-(1H-pyrrol-1-yl)phenyl)-1-((methylthio)methyl)-1H-1,2,3-triazole (2j)

The reaction of $\mathbf{1 j}$ (84.2 mg, $0.5 \mathrm{mmol})$, DPPA (206.2 mg, $0.75 \mathrm{mmol}), \mathrm{Cu}(\mathrm{acac})_{2}(13 \mathrm{mg}, 0.05$ 
mmol) in dry DMSO (2 mL) afforded $94.5 \mathrm{mg}(70 \%)$ of $\mathbf{2 j}$; $2 \mathbf{j}$ : yellow solid; ${ }^{1} \mathrm{H}$ NMR ( $\mathrm{d}_{6}$-DMSO, $400 \mathrm{MHz}$ ): 8.69 (s, 1H), 7.95 (d, $J=8.4 \mathrm{~Hz}, 2 \mathrm{H}), 7.67$ (d, $J=8.4 \mathrm{~Hz}, 2 \mathrm{H}), 7.42(\mathrm{t}, J=2.0 \mathrm{~Hz}, 2 \mathrm{H})$, $6.28(\mathrm{t}, J=2.0 \mathrm{~Hz}, 2 \mathrm{H}), 5.58(\mathrm{~s}, 2 \mathrm{H}), 2.19(\mathrm{~s}, 3 \mathrm{H}) ;{ }^{13} \mathrm{C}$ NMR (d $\left.\mathrm{d}_{6} \mathrm{DMSO}, 100 \mathrm{MHz}\right): 146.7,139.9$, 127.9, 126.9, 121.4, 120.0, 119.3, 111.1, 52.4, 15.1; HRMS: calc. for $\mathrm{C}_{14} \mathrm{H}_{15} \mathrm{~N}_{4} \mathrm{~S}(\mathrm{M}+\mathrm{H})^{+}$, 271.1012 , found, 271.1015 .<smiles>CSCn1cc(-c2cccc(C)c2)nn1</smiles>

\section{1) 1-((Methylthio)methyl)-4-(m-tolyl)-1H-1,2,3-triazole (2k)}

The reaction of $1 \mathbf{k}(58.0 \mathrm{mg}, 0.5 \mathrm{mmol})$, DPPA (206.2 mg, $0.75 \mathrm{mmol}), \mathrm{Cu}(\mathrm{acac})_{2}(13 \mathrm{mg}, 0.05$ mmol) in dry DMSO (2 mL) afforded $78.8 \mathrm{mg}(72 \%)$ of $2 \mathbf{k}$; $2 \mathbf{k}$ : yellow solid; ${ }^{1} \mathrm{H}$ NMR (d $\mathrm{d}_{6}$-DMSO, $\left.400 \mathrm{MHz}\right): 8.62$ (s, 1H), $7.72(\mathrm{~s}, 1 \mathrm{H}), 7.67$ (d, $\left.J=7.6 \mathrm{~Hz}, 1 \mathrm{H}\right), 7.33$ (t, $\left.J=7.6 \mathrm{~Hz}, 1 \mathrm{H}\right)$, $7.15(\mathrm{~d}, J=7.2 \mathrm{~Hz}, 2 \mathrm{H}), 5.56(\mathrm{~s}, 2 \mathrm{H}), 2.36(\mathrm{~s}, 3 \mathrm{H}), 2.18(\mathrm{~s}, 3 \mathrm{H}) ;{ }^{13} \mathrm{C}$ NMR (d $\left.-\mathrm{DMSO}, 100 \mathrm{MHz}\right)$ : $147.4,138.5,130.9,129.2,129.1,126.3,122.9,121.4,52.4,21.5,15.1$; HRMS: calc. for $\mathrm{C}_{11} \mathrm{H}_{14} \mathrm{~N}_{3} \mathrm{~S}(\mathrm{M}+\mathrm{H})^{+}, 220.0903$, found, 220.0904 .<smiles>CSCn1cc(-c2ccc(CO)cc2)nn1</smiles>

\section{I}

\section{2) (3-(1-((Methylthio)methyl)-1H-1,2,3-triazol-4-yl)phenyl)methanol (2I)}

The reaction of 11 (66.0 mg, $0.5 \mathrm{mmol})$, DPPA (206.2 mg, $0.75 \mathrm{mmol}), \mathrm{Cu}(\mathrm{acac})_{2}(13 \mathrm{mg}, 0.05$ mmol) in dry DMSO (2 mL) afforded $70.5 \mathrm{mg}(60 \%)$ of 2l; 2l: yellow solid; ${ }^{1} \mathrm{H}$ NMR $\left(\mathrm{d}_{6}\right.$-DMSO, $400 \mathrm{MHz}): 8.61$ (s, 1H), 7.83 (d, $J=8.0 \mathrm{~Hz}, 2 \mathrm{H}), 7.40$ (d, $J=8.8 \mathrm{~Hz}, 2 \mathrm{H}), 5.56$ (s, 2H), 5.20 (t, $J$ $=5.6 \mathrm{~Hz}, 1 \mathrm{H}), 4.53(\mathrm{~d}, J=5.6 \mathrm{~Hz}, 2 \mathrm{H}), 2.19(\mathrm{~s}, 3 \mathrm{H}) ;{ }^{13} \mathrm{C}$ NMR $\left(\mathrm{d}_{6}-\mathrm{DMSO}, 100 \mathrm{MHz}\right): 147.3$, 142.8, 129.4, 127.4, 125.5, 121.2, 63.1, 52.3, 15.1; HRMS: calc. for $\mathrm{C}_{11} \mathrm{H}_{14} \mathrm{~N}_{3} \mathrm{OS}(\mathrm{M}+\mathrm{H})^{+}$, 236.0852, found, 236.0854 .<smiles>CSCn1cc(-c2cccc(O)c2)nn1</smiles>

\section{3) 3-(1-((Methylthio)methyl)-1H-1,2,3-triazol-4-yl)phenol (2m)}

The reaction of $1 \mathrm{~m}(59.0 \mathrm{mg}, 0.5 \mathrm{mmol})$, DPPA (206.2 mg, $0.75 \mathrm{mmol}), \mathrm{Cu}(\mathrm{acac})_{2}(13 \mathrm{mg}, 0.05$ mmol) in dry DMSO (2 mL) afforded $84.0 \mathrm{mg}(76 \%)$ of $\mathbf{2 m}$; $2 \mathbf{m}$ : yellow solid; ${ }^{1} \mathrm{H}$ NMR (d6-DMSO, $400 \mathrm{MHz}$ ): 9.53 (brs, 1H), 8.56 (s, 1H), 7.32-7.22 (m, 3H), 6.75 (d, J = 7.6 Hz, 1H), 5.54 (s, 2H), 2.18 (s, 3H); ${ }^{13} \mathrm{C}$ NMR (d 6 -DMSO, $100 \mathrm{MHz}$ ): 158.2, 147.4, 132.2, 130.4, 121.4, 116.6, 115.5, 112.5, 52.4, 15.1; HRMS: calc. for $\mathrm{C}_{10} \mathrm{H}_{12} \mathrm{~N}_{3} \mathrm{OS}(\mathrm{M}+\mathrm{H})^{+}, 222.0696$, found, 222.0696 . 
<smiles>CSCn1cc(-c2ccccc2C(F)(F)F)nn1</smiles>

14) 1-((Methylthio)methyl)-4-(2-(trifluoromethyl)phenyl)-1H-1,2,3-triazole (2n)

The reaction of $1 \mathrm{n}(85.3 \mathrm{mg}, 0.5 \mathrm{mmol})$, DPPA (206.2 mg, $0.75 \mathrm{mmol}), \mathrm{Cu}(\mathrm{acac})_{2}(13 \mathrm{mg}, 0.05$ mmol) in dry DMSO $(2 \mathrm{~mL})$ afforded $101.2 \mathrm{mg}(74 \%)$ of $\mathbf{2 n}$; $2 \mathbf{n}$ : yellow solid; ${ }^{1} \mathrm{H}$ NMR (d $\mathrm{d}_{6}$-DMSO, $\left.400 \mathrm{MHz}\right): 8.40$ (s, 1H), 7.84 (t, $\left.J=8.0 \mathrm{~Hz}, 2 \mathrm{H}\right), 7.77$ (t, $\left.J=7.6 \mathrm{~Hz}, 1 \mathrm{H}\right), 7.63$ (t, $J=$ $7.6 \mathrm{~Hz}, 1 \mathrm{H}), 5.63(\mathrm{~s}, 2 \mathrm{H}), 2.18(\mathrm{~s}, 3 \mathrm{H}) ;{ }^{13} \mathrm{C}$ NMR (d 6 -DMSO, $\left.100 \mathrm{MHz}\right): 144.0,133.1,132.2$, 129.8 (q, $1.9 \mathrm{~Hz}), 129.3,126.9$ (q, $29.8 \mathrm{~Hz}), 126.7$ (q, 5.4 Hz), 124.5 (q, J = 272.0 Hz), 124.2 (q, 4.0 Hz), 52.3, 14.9; HRMS: calc. for $\mathrm{C}_{11} \mathrm{H}_{11} \mathrm{~F}_{3} \mathrm{~N}_{3} \mathrm{~S}(\mathrm{M}+\mathrm{H})^{+}$, 274.0620, found, 274.0623.<smiles>CSCn1cc(-c2ccccc2C)nn1</smiles>

\section{5) 1-((Methylthio)methyl)-4-(o-tolyl)-1H-1,2,3-triazole (2o)}

The reaction of 10 (58.0 mg, $0.5 \mathrm{mmol})$, DPPA (206.2 mg, $0.75 \mathrm{mmol}), \mathrm{Cu}(\mathrm{acac})_{2}(13 \mathrm{mg}, 0.05$ $\mathrm{mmol})$ in dry DMSO $(2 \mathrm{~mL})$ afforded $91.0 \mathrm{mg}(83 \%)$ of 2o; 2o: white solid; ${ }^{1} \mathrm{H}$ NMR $\left(\mathrm{d}_{6}\right.$-DMSO, $400 \mathrm{MHz}): 8.46(\mathrm{~s}, 1 \mathrm{H}), 7.76(\mathrm{t}, J=6.0 \mathrm{~Hz}, 1 \mathrm{H}), 7.32-7.26(\mathrm{~m}, 3 \mathrm{H}), 5.58(\mathrm{~s}, 2 \mathrm{H}), 2.44(\mathrm{~s}, 3 \mathrm{H})$, $2.20(\mathrm{~s}, 3 \mathrm{H}) ;{ }^{13} \mathrm{C}$ NMR (d 6 -DMSO, $\left.100 \mathrm{MHz}\right): 146.4,135.4,131.3,130.3,128.7,128.4,126.5$, 123.4, 52.2, 21.6, 15.2; HRMS: calc. for $\mathrm{C}_{11} \mathrm{H}_{14} \mathrm{~N}_{3} \mathrm{~S}(\mathrm{M}+\mathrm{H})^{+}, 220.0903$, found, 220.0904 .<smiles>CSCn1cc(-c2ccccc2Cl)nn1</smiles>

\section{6) 4-(2-Chlorophenyl)-1-((methylthio)methyl)-1H-1,2,3-triazole (2p)}

The reaction of 1p (68.4 mg, $0.5 \mathrm{mmol})$, DPPA (206.2 mg, $0.75 \mathrm{mmol}), \mathrm{Cu}(\mathrm{acac})_{2}(13 \mathrm{mg}, 0.05$ mmol) in dry DMSO (2 mL) afforded $84.8 \mathrm{mg}(71 \%)$ of $\mathbf{2 p}$; 2p: white solid; ${ }^{1} \mathrm{H}$ NMR ( $\mathrm{d}_{6}$-DMSO, $400 \mathrm{MHz}$ ): $8.72(\mathrm{~s}, 1 \mathrm{H}), 8.10$ (d, $J=7.6 \mathrm{~Hz}, 1 \mathrm{H}), 7.56(\mathrm{~d}, J=7.6 \mathrm{~Hz}, 1 \mathrm{H}), 7.46$ (t, $J=7.6 \mathrm{~Hz}, 1 \mathrm{H})$, 7.39 (t, $J=7.6 \mathrm{~Hz}, 1 \mathrm{H}), 5.62$ (s, 2H), 2.20 (s, 3H); ${ }^{13} \mathrm{C}$ NMR (d 6 -DMSO, $\left.100 \mathrm{MHz}\right): 143.4,130.8$, 130.7, 130.0, 129.9, 129.5, 128.0, 124.3, 52.3, 15.1; HRMS: calc. for $\mathrm{C}_{10} \mathrm{H}_{11} \mathrm{ClN}_{3} \mathrm{~S}(\mathrm{M}+\mathrm{H})^{+}$, 240.0357 , found, 240.0360 .<smiles>CSCn1cc(-c2cc(F)cc(F)c2)nn1</smiles>

17) 4-(3,5-Difluorophenyl)-1-((methylthio)methyl)-1H-1,2,3-triazole (2q)

The reaction of 1q $(69.4 \mathrm{mg}, 0.5 \mathrm{mmol})$, DPPA $(206.2 \mathrm{mg}, 0.75 \mathrm{mmol}), \mathrm{Cu}(\mathrm{acac})_{2}(13 \mathrm{mg}, 0.05$ 
mmol) in dry DMSO $(2 \mathrm{~mL})$ afforded $101.0 \mathrm{mg}(84 \%)$ of $\mathbf{2 q}$; $2 \mathbf{2 q}$ : yellow solid; ${ }^{1} \mathrm{H}$ NMR (d $\mathrm{d}_{6}$-DMSO, $\left.400 \mathrm{MHz}\right): 8.80(\mathrm{~s}, 1 \mathrm{H}), 7.63-7.56(\mathrm{~m}, 2 \mathrm{H}), 7.20-7.13(\mathrm{~m}, 1 \mathrm{H}), 5.59(\mathrm{~s}, 2 \mathrm{H}), 2.18(\mathrm{~s}$, $3 \mathrm{H}) ;{ }^{13} \mathrm{C}$ NMR (d 6 -DMSO, $\left.100 \mathrm{MHz}\right): 163.4$ (d, $\left.J=244.3 \mathrm{~Hz}\right), 163.3$ (d, $\left.J=244.3 \mathrm{~Hz}\right), 145.4$ (t, $J=3.1 \mathrm{~Hz}), 134.5(\mathrm{t}, J=0.8 \mathrm{~Hz}), 122.8,108.7(\mathrm{dd}, J=19.3 \mathrm{~Hz}, J=8.2 \mathrm{~Hz}), 103.6(\mathrm{t}, J=26.1 \mathrm{~Hz})$, 52.6, 15.1; HRMS: calc. for $\mathrm{C}_{10} \mathrm{H}_{10} \mathrm{~F}_{2} \mathrm{~N}_{3} \mathrm{~S}(\mathrm{M}+\mathrm{H})^{+}, 242.0558$, found, 242.0559 .<smiles>CSCn1cc(-c2ccc3ccccc3c2)nn1</smiles>

18) 1-((Methylthio)methyl)-4-(naphthalen-2-yl)-1H-1,2,3-triazole (2r)

The reaction of $1 \mathbf{r}(76.0 \mathrm{mg}, 0.5 \mathrm{mmol})$, DPPA $(206.2 \mathrm{mg}, 0.75 \mathrm{mmol}), \mathrm{Cu}(\mathrm{acac})_{2}(13 \mathrm{mg}, 0.05$ mmol) in dry DMSO $(2 \mathrm{~mL})$ afforded $112.0 \mathrm{mg}(88 \%)$ of $2 \mathbf{r}$; $2 \mathbf{r}$ : yellow solid; ${ }^{1} \mathrm{H}$ NMR $\left(\mathrm{d}_{6}\right.$-DMSO, $\left.400 \mathrm{MHz}\right): 8.79$ (s, 1H), $8.46(\mathrm{~s}, 1 \mathrm{H}), 8.07-7.93(\mathrm{~m}, 3 \mathrm{H}), 7.91(\mathrm{~d}, J=1.6 \mathrm{~Hz}, 1 \mathrm{H})$, 7.56-7.49 (m, 2H), $5.62(\mathrm{~s}, 2 \mathrm{H}), 2.21(\mathrm{~s}, 3 \mathrm{H}) ;{ }^{13} \mathrm{C}$ NMR (d 6 -DMSO, $\left.100 \mathrm{MHz}\right): 147.4,133.7$, 133.1, 129.0, 128.5, 128.2, 127.1, 126.7, 124.2, 124.1, 121.9, 52.5, 15.1; HRMS: calc. for $\mathrm{C}_{14} \mathrm{H}_{14} \mathrm{~N}_{3} \mathrm{~S}(\mathrm{M}+\mathrm{H})^{+}, 256.0903$, found, 256.0905.<smiles>CSCn1cc(-c2cccc3ccccc23)nn1</smiles>

2s

19) 1-((Methylthio)methyl)-4-(naphthalen-1-yl)-1H-1,2,3-triazole (2s)

The reaction of $1 \mathrm{~s}$ (76.0 mg, $0.5 \mathrm{mmol})$, DPPA (206.2 mg, $0.75 \mathrm{mmol}), \mathrm{Cu}(\mathrm{acac})_{2}(13 \mathrm{mg}, 0.05$ $\mathrm{mmol})$ in dry DMSO $(2 \mathrm{~mL})$ heated for $36 \mathrm{~h}$, afforded $80.3 \mathrm{mg}(63 \%)$ of $2 \mathrm{~s}$; $2 \mathrm{~s}$ : yellow solid; ${ }^{1} \mathrm{H}$ NMR (d 6 -DMSO, $400 \mathrm{MHz}): 8.67(\mathrm{~s}, 1 \mathrm{H}), 8.49$ (d, $J=5.6 \mathrm{~Hz}, 1 \mathrm{H}), 7.97(\mathrm{~d}, J=8.0 \mathrm{~Hz}, 2 \mathrm{H}), 7.80$ $(\mathrm{d}, J=5.6 \mathrm{~Hz}, 1 \mathrm{H}), 7.59-7.57(\mathrm{~m}, 3 \mathrm{H}), 5.66(\mathrm{~s}, 2 \mathrm{H}), 2.55(\mathrm{~s}, 3 \mathrm{H}) ;{ }^{13} \mathrm{C}$ NMR $\left(\mathrm{d}_{6}-\mathrm{DMSO}, 100\right.$ MHz): 146.5, 134.0, 130.8, 129.1, 128.9, 128.2, 127.4, 127.2, 126.6, 126.0, 125.8, 124.2, 52.4, 15.3; HRMS: calc. for $\mathrm{C}_{14} \mathrm{H}_{14} \mathrm{~N}_{3} \mathrm{~S}(\mathrm{M}+\mathrm{H})^{+}, 256.0903$, found, 256.0904 .<smiles>[Z20]c1ccsc1-c1cn(CSC)nn1</smiles>

\section{0) 1-((Methylthio)methyl)-4-(thiophen-3-yl)-1H-1,2,3-triazole (2t)}

The reaction of 1t $(54.1 \mathrm{mg}, 0.5 \mathrm{mmol})$, DPPA (206.2 mg, $0.75 \mathrm{mmol}), \mathrm{Cu}(\mathrm{acac})_{2}(13 \mathrm{mg}, 0.05$ $\mathrm{mmol})$ in dry DMSO $(2 \mathrm{~mL})$ afforded $95.0 \mathrm{mg}(90 \%)$ of $\mathbf{2 t}$; $2 \mathbf{t}$ : yellow solid; ${ }^{1} \mathrm{H}$ NMR $\left(\mathrm{d}_{6}\right.$-DMSO, $400 \mathrm{MHz}): 8.51(\mathrm{~s}, 1 \mathrm{H}), 7.88(\mathrm{~d}, J=1.2 \mathrm{~Hz}, 1 \mathrm{H}), 7.64-7.62(\mathrm{~m}, 1 \mathrm{H}), 7.55(\mathrm{~d}, J=4.4 \mathrm{~Hz}, 1 \mathrm{H})$, $5.56(\mathrm{~s}, 2 \mathrm{H}), 2.17$ (s, 3H); ${ }^{13} \mathrm{C}$ NMR (d $\mathrm{d}_{6}$-DMSO, $\left.100 \mathrm{MHz}\right): 143.8,132.3,127.5,126.3,121.5$, 121.1, 52.3, 15.0; HRMS: calc. for $\mathrm{C}_{8} \mathrm{H}_{10} \mathrm{~N}_{3} \mathrm{~S}_{2}(\mathrm{M}+\mathrm{H})^{+}, 212.0311$, found, 212.0310 . 
<smiles>CSCn1cc(C2=CCCCC2)nn1</smiles>

\section{$2 u$}

\section{1) 4-(Cyclohex-1-en-1-yl)-1-((methylthio)methyl)-1H-1,2,3-triazole (2u)}

The reaction of $1 \mathbf{u}(53.0 \mathrm{mg}, 0.5 \mathrm{mmol})$, DPPA (206.2 mg, $0.75 \mathrm{mmol}), \mathrm{Cu}(\mathrm{acac})_{2}(13 \mathrm{mg}, 0.05$ mmol) in dry DMSO $(2 \mathrm{~mL})$ afforded $77.3 \mathrm{mg}(74 \%)$ of $\mathbf{2 u}$; $2 \mathbf{u}$ : yellow solid; ${ }^{1} \mathrm{H}$ NMR (d $\mathrm{d}_{6}$-DMSO, $\left.400 \mathrm{MHz}\right): 8.12(\mathrm{~s}, 1 \mathrm{H}), 6.44-6.41(\mathrm{~m}, 1 \mathrm{H}), 5.46(\mathrm{~s}, 2 \mathrm{H}), 2.34-2.31(\mathrm{~m}, 2 \mathrm{H}), 2.17-2.12$ (m, 5H), 1.72-1.66 (m ,2H), 1.63-1.58 (m, 2H); ${ }^{13} \mathrm{C}$ NMR (d 6 -DMSO, $\left.100 \mathrm{MHz}\right): 149.0,127.8$, 124.2, 119.9, 52.1, 26.2, 25.1, 22.5, 22.3, 15.0; HRMS: calc. for $\mathrm{C}_{10} \mathrm{H}_{16} \mathrm{~N}_{3} \mathrm{~S}(\mathrm{M}+\mathrm{H})^{+}, 210.1059$, found, 210.1061 .<smiles>CSCn1cc(Cc2ccccc2)nn1</smiles>

\section{2) 4-Benzyl-1-((methylthio)methyl)-1H-1,2,3-triazole (2v)}

The reaction of $1 \mathbf{v}(58.0 \mathrm{mg}, 0.5 \mathrm{mmol})$, DPPA (206.2 mg, $0.75 \mathrm{mmol}), \mathrm{Cu}(\mathrm{acac})_{2}(13 \mathrm{mg}, 0.05$ $\mathrm{mmol})$ in dry DMSO $(2 \mathrm{~mL})$ afforded $62.4 \mathrm{mg}(57 \%)$ of $\mathbf{2 v}$; $2 \mathbf{v}$ : yellow solid; ${ }^{1} \mathrm{H}$ NMR $\left(\mathrm{d}_{6}\right.$-DMSO, $400 \mathrm{MHz}): 7.90$ (s, 1H), 7.32-7.18 (m, 5H), $5.46(\mathrm{~s}, 2 \mathrm{H}), 4.01(\mathrm{~s}, 2 \mathrm{H}), 2.11$ (s, 3H); ${ }^{13} \mathrm{C}$ NMR $\left(\mathrm{d}_{6}\right.$-DMSO, $\left.100 \mathrm{MHz}\right):$ 147.1, 140.0, 129.0, 128.9, 126.7, 122.6, 51.9, 31.7, 15.0; HRMS: calc. for $\mathrm{C}_{11} \mathrm{H}_{14} \mathrm{~N}_{3} \mathrm{~S}(\mathrm{M}+\mathrm{H})^{+}, 220.0903$, found, 220.0905 .

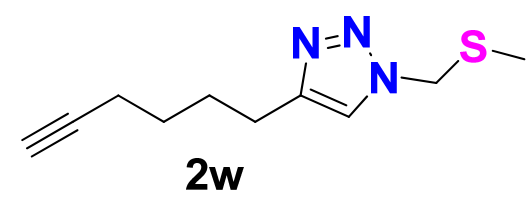

\section{3) 4-(Hex-5-yn-1-yl)-1-((methylthio)methyl)-1H-1,2,3-triazole (2w)}

The reaction of $1 \mathrm{w}(54.0 \mathrm{mg}, 0.5 \mathrm{mmol})$, DPPA (206.2 mg, $0.75 \mathrm{mmol}), \mathrm{Cu}(\mathrm{acac})_{2}(13 \mathrm{mg}, 0.05$ mmol) in dry DMSO $(2 \mathrm{~mL})$ afforded $69.0 \mathrm{mg}(66 \%)$ of $2 \mathrm{w}$; $2 \mathrm{w}$ : yellow solid; ${ }^{1} \mathrm{H}$ NMR (d $\mathrm{d}_{6}$-DMSO, $\left.400 \mathrm{MHz}\right): 7.91$ (s, 1H), 5.45 (s, 2H), 2.73 (d, $\left.J=2.4 \mathrm{~Hz}, 1 \mathrm{H}\right), 2.63$ (d, $J=8.0 \mathrm{~Hz}$, $2 \mathrm{H}), 2.20-2.16(\mathrm{~m}, 2 \mathrm{H}), 2.11(\mathrm{~s}, 3 \mathrm{H}), 1.72-1.64(\mathrm{~m}, 2 \mathrm{H}), 1.52-1.46(\mathrm{~m}, 2 \mathrm{H}),{ }^{13} \mathrm{C}$ NMR $\left(\mathrm{d}_{6}\right.$-DMSO, $100 \mathrm{MHz})$ : 147.6, 122.0, 84.8, 71.6, 51.9, 28.4, 27.98, 24.9, 17.8, 15.0; HRMS: calc. for $\mathrm{C}_{10} \mathrm{H}_{16} \mathrm{~N}_{3} \mathrm{~S}(\mathrm{M}+\mathrm{H})^{+}, 210.1059$, found, 210.1059 .<smiles>[R20]c1c(C(C)=O)nnn1CSC</smiles>

\section{4) 1-(1-((Methylthio)methyl)-5-phenyl-1H-1,2,3-triazol-4-yl)ethanone (2x)}

The reaction of $1 \mathbf{x}(72.0 \mathrm{mg}, 0.5 \mathrm{mmol})$, DPPA (206.2 mg, $0.75 \mathrm{mmol}), \mathrm{Cu}(\mathrm{acac})_{2}(13 \mathrm{mg}, 0.05$ $\mathrm{mmol})$ in dry DMSO $(2 \mathrm{~mL})$ afforded $44.5 \mathrm{mg}(36 \%)$ of $\mathbf{2 x}$; 2x: yellow oil; ${ }^{1} \mathrm{H} \mathrm{NMR}\left(\mathrm{CDCl}_{3}, 400\right.$ MHz): 7.53-7.45 (m, 5H), 5.20 (s, 2H), 2.69 (s, 3H), 2.30 (s, 3H); ${ }^{13} \mathrm{C} \mathrm{NMR}\left(\mathrm{CDCl}_{3}, 400 \mathrm{MHz}\right.$, $100 \mathrm{MHz}$ ): 192.6, 143.8, 139.0, 130.3, 130.0, 128.8, 125.6, 50.8, 28.0, 15.7; HRMS: calc. for $\mathrm{C}_{12} \mathrm{H}_{13} \mathrm{~N}_{3} \mathrm{NaOS}(\mathrm{M}+\mathrm{H})^{+}, 270.0672$, found, 270.0666 . 
<smiles></smiles>

The reaction of $\mathbf{1 x}$ (72.0 mg, $0.5 \mathrm{mmol})$, DPPA (206.2 mg, $0.75 \mathrm{mmol}), \mathrm{Cu}(\mathrm{acac})_{2}(13 \mathrm{mg}, 0.05$ mmol) in dry DMSO (2 mL) afforded $14.8 \mathrm{mg}(12 \%)$ of $\mathbf{2} \mathbf{x}$; $2 \mathbf{2 x}^{\prime}$ : yellow oil; ${ }^{1} \mathrm{H}$ NMR $\left(\mathrm{CDCl}_{3}\right.$, $400 \mathrm{MHz})$ : 7.56-7.49 (m, 5H), 5.69 (s, 2H), 2.28 (s, 3H), $2.22(\mathrm{~s}, 3 \mathrm{H}) ;{ }^{13} \mathrm{C} \mathrm{NMR}\left(\mathrm{CDCl}_{3}, 400\right.$ MHz, $100 \mathrm{MHz}): 190.4,150.9,130.4,130.2,129.59,129.56,128.8,53.9,30.2,15.0$; HRMS: calc. for $\mathrm{C}_{12} \mathrm{H}_{14} \mathrm{~N}_{3} \mathrm{OS}(\mathrm{M}+\mathrm{H})^{+}, 248.08521$, found, 248.08574 .<smiles>COC(=O)c1nnn(CSC)c1C(=O)OC</smiles>

$2 y$

25) Dimethyl 1-((methylthio)methyl)-1H-1,2,3-triazole-4,5-dicarboxylate (2y)

The reaction of $1 \mathbf{y}(43.0 \mathrm{mg}, 0.3 \mathrm{mmol})$, DPPA $(206.2 \mathrm{mg}, 0.75 \mathrm{mmol}), \mathrm{Cu}(\mathrm{acac})_{2}(7.8 \mathrm{mg}, 0.03$ mmol) in dry DMSO $(2 \mathrm{~mL})$ afforded $35.3 \mathrm{mg}(48 \%)$ of $2 \mathbf{y}$; $2 \mathbf{y}$ : yellow solid; ${ }^{1} \mathrm{H}$ NMR $\left(\mathrm{CDCl}_{3}\right.$, $400 \mathrm{MHz}$ ): 5.63 (s, 2H), 4.02 (s, 3H), 3.99 (s, 3H), 2.15 (s, 3H); ${ }^{13} \mathrm{C} \mathrm{NMR}\left(\mathrm{CDCl}_{3}, 100 \mathrm{MHz}\right.$ ): 160.2, 158.6, 140.6, 129.0, 59.0, 53.4, 52.7, 14.8; HRMS: calc. for $\mathrm{C}_{8} \mathrm{H}_{12} \mathrm{~N}_{3} \mathrm{O}_{4} \mathrm{~S}(\mathrm{M}+\mathrm{H})^{+}$, 246.0543 , found, 246.0543 .<smiles>CSCn1nnc(C(=O)c2ccccc2)c1-c1ccccc1</smiles>

$4 a$

26) (1-((Methylthio)methyl)-5-phenyl-1H-1,2,3-triazol-4-yl)(phenyl)methanone (4a)

The reaction of 3a $(62.0 \mathrm{mg}, 0.3 \mathrm{mmol})$, DPPA (206.2 mg, $0.75 \mathrm{mmol}), \mathrm{Cu}(\mathrm{acac})_{2}(7.8 \mathrm{mg}, 0.05$ $\mathrm{mmol})$ in dry DMSO (2 mL) afforded $46.3 \mathrm{mg}(50 \%)$ of $\mathbf{4 a}$; $4 \mathbf{a}$ : yellow oil; ${ }^{1} \mathrm{H} \mathrm{NMR}\left(\mathrm{CDCl}_{3}, 400\right.$ MHz): 8.27-8.25 (m, 2H), 7.60-7.56 (m, 6H), 7.52-7.46 (m, 2H), $5.26(\mathrm{~s}, 2 \mathrm{H}), 2.33(\mathrm{~s}, 3 \mathrm{H}) ;{ }^{13} \mathrm{C}$ NMR (CDCl $100 \mathrm{MHz})$ : 186.3, 143.9, 141.3, 137.1, 133.0, 130.6, 130.2, 130.0, 128.8, 128.2, 126.0, 51.0, 15.9; HRMS: calc. for $\mathrm{C}_{17} \mathrm{H}_{16} \mathrm{~N}_{3} \mathrm{OS}(\mathrm{M}+\mathrm{H})^{+}, 310.1009$, found, 310.1010 .<smiles>CSCn1nnc(C(=O)c2ccccc2)c1-c1ccc(Cl)cc1</smiles>

27) (5-(4-Chlorophenyl)-1-((methylthio)methyl)-1H-1,2,3-triazol-4-yl)(phenyl)methanone 
(4b)

The reaction of $3 \mathbf{b}(73.0 \mathrm{mg}, 0.3 \mathrm{mmol})$, DPPA $(0.6 \mathrm{mmol}, 165.0 \mathrm{mg}), \mathrm{Cu}(\mathrm{acac})_{2}(7.8 \mathrm{mg}, 0.03$ mmol) in dry DMSO (2 mL) afforded $46.0 \mathrm{mg}(45 \%)$ of $\mathbf{4 b}$; $\mathbf{4 b}$ : yellow oil; ${ }^{1} \mathrm{H}$ NMR $\left(\mathrm{d}_{6}\right.$-DMSO, $400 \mathrm{MHz}$ ): 8.09 (d, $J=7.6 \mathrm{~Hz}, 2 \mathrm{H}), 7.67(\mathrm{t}, J=7.2 \mathrm{~Hz}, 1 \mathrm{H}), 7.61-7.60(\mathrm{~m}, 4 \mathrm{H}), 7.55$ (t, $J=7.6$ $\mathrm{Hz}, 2 \mathrm{H}), 5.49$ (s, 2H), 2.13 (s, 3H); ${ }^{13} \mathrm{C} \mathrm{NMR}\left(\mathrm{CDCl}_{3}, 100 \mathrm{MHz}\right): 186.4,143.8,140.2,137.3$, 135.4, 133.7, 132.4, 130.6, 129.2, 128.8, 125.3, 51.3, 15.3; HRMS: calc. for $\mathrm{C}_{17} \mathrm{H}_{15} \mathrm{ClN}_{3} \mathrm{OS}$ $(\mathrm{M}+\mathrm{H})^{+}, 344.0619$, found, 344.0613 .<smiles></smiles>

28) (1-((Methylthio)methyl)-5-(4-nitrophenyl)-1H-1,2,3-triazol-4-yl)(phenyl)methanone (4c) The reaction of 3c $(76.0 \mathrm{mg}, 0.3 \mathrm{mmol})$, DPPA $(0.6 \mathrm{mmol}, 165.0 \mathrm{mg}), \mathrm{Cu}(\mathrm{acac})_{2}(7.8 \mathrm{mg}, 0.03$ $\mathrm{mmol})$ in dry DMSO $(2 \mathrm{~mL})$ afforded $58.4 \mathrm{mg}(55 \%)$ of $4 \mathrm{c}$; $4 \mathrm{c}$ : yellow oil; ${ }^{1} \mathrm{H}$ NMR $\left(\mathrm{d}_{6}\right.$-DMSO, $400 \mathrm{MHz}): 8.37$ (dt, $J=8.8 \mathrm{~Hz}, J=2.0 \mathrm{~Hz}, 2 \mathrm{H}), 8.15-8.12(\mathrm{~m}, 2 \mathrm{H}), 7.89$ (dt, $J=8.8 \mathrm{~Hz}, J=2.0$ $\mathrm{Hz}, 2 \mathrm{H}), 7.70-7.66(\mathrm{~m}, 1 \mathrm{H}), 7.58-7.54(\mathrm{~m}, 2 \mathrm{H}), 5.53(\mathrm{~s}, 2 \mathrm{H}), 2.13(\mathrm{~s}, 3 \mathrm{H}) ;{ }^{13} \mathrm{C} \mathrm{NMR} \mathrm{d}_{6}$-DMSO, $400 \mathrm{MHz})$ : 186.3, 148.7, 144.3, 139.5, 137.0, 133.8, 133.3, 132.1, 130.6, 128.8, 124.0, 51.5, 15.2; HRMS: calc. for $\mathrm{C}_{17} \mathrm{H}_{15} \mathrm{~N}_{4} \mathrm{O}_{3} \mathrm{~S}(\mathrm{M}+\mathrm{H})^{+}, 355.0859$, found, 355.0859 .<smiles>CCOC(=O)c1nnn(CSC)c1C(=O)c1ccccc1</smiles>

29) Ethyl 4-benzoyl-1-((methylthio)methyl)-1H-1,2,3-triazole-5-carboxylate (4d)

The reaction of $3 \mathbf{d}(61.2 \mathrm{mg}, 0.5 \mathrm{mmol})$, DPPA $(0.6 \mathrm{mmol}, 165.0 \mathrm{mg}), \mathrm{Cu}(\mathrm{acac})_{2}(13 \mathrm{mg}, 0.05$ $\mathrm{mmol})$ in dry DMSO $(2 \mathrm{~mL})$ afforded $46.7 \mathrm{mg}(51 \%)$ of $\mathbf{4 d}$; $\mathbf{4 d}$ : yellow oil; ${ }^{1} \mathrm{H}$ NMR $\left(\mathrm{d}_{6}\right.$-DMSO, $00 \mathrm{MHz}$ ): 7.92 (d, $J=7.2 \mathrm{~Hz}, 2 \mathrm{H}), 7.74(\mathrm{t}, J=7.2 \mathrm{~Hz}, 1 \mathrm{H}), 7.60$ (t, $J=7.6 \mathrm{~Hz}, 2 \mathrm{H}), 5.80$ (s, 2H), 4.18 (q, $J=7.6 \mathrm{~Hz}, 2 \mathrm{H}), 2.21(\mathrm{~s}, 3 \mathrm{H}), 0.99(\mathrm{t}, J=7.6 \mathrm{~Hz}, 3 \mathrm{H}) ;{ }^{13} \mathrm{C}$ NMR (d $\left.\mathrm{d}_{6}-\mathrm{DMSO}, 100 \mathrm{MHz}\right)$ : 187.3, 157.9, 147.5, 136.5, 134.9, 130.2, 129.4, 128.4, 62.9, 53.6, 15.2, 13.7; HRMS: calc. for $\mathrm{C}_{14} \mathrm{H}_{16} \mathrm{~N}_{3} \mathrm{O}_{3} \mathrm{~S}(\mathrm{M}+\mathrm{H})^{+}, 306.0907$, found, 306.0911.<smiles>CCSCn1cc(-c2ccc(OC)cc2)nn1</smiles>

30) 1-((Ethylthio)methyl)-4-(4-methoxyphenyl)-1H-1,2,3-triazole (6a) 
The reaction of 1a (39.6 mg, $0.3 \mathrm{mmol})$, DPPA (165.0 mg, $0.6 \mathrm{mmol}), \mathrm{Cu}(\mathrm{acac})_{2}(7.6 \mathrm{mg}, 0.03$ $\mathrm{mmol})$ in dry $\mathrm{CH}_{3} \mathrm{~S}(=\mathrm{O}) \mathrm{CH}_{2} \mathrm{CH}_{3}(0.5 \mathrm{~mL})$ afforded $48.6 \mathrm{mg}(65 \%)$ of $\mathbf{6 a} ; \mathbf{6 a}$ : white solid; ${ }^{1} \mathrm{H}$ NMR (d $\mathrm{d}_{6}$-DMSO, $\left.400 \mathrm{MHz}\right): 8.54(\mathrm{~s}, 1 \mathrm{H}), 7.80(\mathrm{~d}, J=8.8 \mathrm{~Hz}, 2 \mathrm{H}), 7.01(\mathrm{~d}, J=8.8 \mathrm{~Hz}, 2 \mathrm{H}), 5.58$ (s, 2H), 3.79 (s, 3H), 2.62 (q, $J=7.2 \mathrm{~Hz}, 2 \mathrm{H}), 1.18$ (t, $J=7.2 \mathrm{~Hz}, 3 \mathrm{H}) ;{ }^{13} \mathrm{C}$ NMR (d $\mathrm{d}_{6}$-DMSO, 100 $\mathrm{MHz})$ : 159.6, 147.3, 127.1, 123.6, 120.5, 114.8, 55.6, 50.1, 25.6, 14.7; HRMS: calc. for $\mathrm{C}_{12} \mathrm{H}_{16} \mathrm{~N}_{3} \mathrm{OS}(\mathrm{M}+\mathrm{H})^{+}, 250.1009$, found, 250.1012 .<smiles>CCCSCn1cc(-c2ccc(OC)cc2)nn1</smiles>

31) 4-(4-Methoxyphenyl)-1-((propylthio)methyl)-1H-1,2,3-triazole (6b)

The reaction of 1a (39.6 mg, $0.3 \mathrm{mmol})$, DPPA (165.0 mg, $0.6 \mathrm{mmol}), \mathrm{Cu}(\mathrm{acac})_{2}(7.6 \mathrm{mg}, 0.03$ $\mathrm{mmol})$ in dry $\mathrm{CH}_{3} \mathrm{~S}(=\mathrm{O}) \mathrm{CH}_{2} \mathrm{CH}_{2} \mathrm{CH}_{3}(0.5 \mathrm{~mL})$ afforded $40.2 \mathrm{mg}(51 \%)$ of $\mathbf{6 b} ; \mathbf{6 b}$ : white solid; ${ }^{1} \mathrm{H}$ NMR (d 6 -DMSO, $400 \mathrm{MHz}): 8.54(\mathrm{~s}, 1 \mathrm{H}), 7.79(\mathrm{~d}, J=8.8 \mathrm{~Hz}, 2 \mathrm{H}), 7.01(\mathrm{~d}, J=8.8 \mathrm{~Hz}, 2 \mathrm{H}), 5.55$ (s, 2H), 3.79 (s, 3H), 2.60 (t, $J=7.2 \mathrm{~Hz}, 2 \mathrm{H}), 1.56-1.50(\mathrm{~m}, 2 \mathrm{H}), 0.89(\mathrm{t}, J=7.2 \mathrm{~Hz}, 3 \mathrm{H}) ;{ }^{13} \mathrm{C}$ NMR (d $\mathrm{d}_{6}$-DMSO, $\left.100 \mathrm{MHz}\right): 159.6,147.3,127.1,123.6,120.5,114.8,55.6,50.5,33.5,22.4,13.5$; HRMS: calc. for $\mathrm{C}_{13} \mathrm{H}_{18} \mathrm{~N}_{3} \mathrm{OS}(\mathrm{M}+\mathrm{H})^{+}, 264.1165$, found, 264.1168.<smiles>CCC(C)SCn1cc(-c2ccc(OC)cc2)nn1</smiles>

\section{2) 1-((sec-Butylthio)methyl)-4-(4-methoxyphenyl)-1H-1,2,3-triazole (6c)}

The reaction of 1a (39.6 mg, $0.3 \mathrm{mmol})$, DPPA (165.0 mg, $0.6 \mathrm{mmol}), \mathrm{Cu}(\mathrm{acac})_{2}(7.6 \mathrm{mg}, 0.03$ mmol) in dry $\mathrm{CH}_{3} \mathrm{~S}(=\mathrm{O}) \mathrm{CH}\left(\mathrm{CH}_{3}\right) \mathrm{CH}_{2} \mathrm{CH}_{3}(0.5 \mathrm{~mL})$ afforded $55.7 \mathrm{mg}(67 \%)$ of $\mathbf{6 c} ; \mathbf{6 c}$ : white solid; ${ }^{1} \mathrm{H}$ NMR (d $\mathrm{d}_{6}$-DMSO, $\left.400 \mathrm{MHz}\right): 8.55(\mathrm{~s}, 1 \mathrm{H}), 7.80(\mathrm{~d}, J=8.8 \mathrm{~Hz}, 2 \mathrm{H}), 7.01(\mathrm{~d}, J=8.8 \mathrm{~Hz}, 2 \mathrm{H})$, $5.58(\mathrm{~s}, 2 \mathrm{H}), 3.78(\mathrm{~s}, 3 \mathrm{H}), 2.85(\mathrm{q}, J=6.8 \mathrm{~Hz}, 1 \mathrm{H}), 1.55-1.43(\mathrm{~m}, 2 \mathrm{H}), 1.20(\mathrm{~d}, J=6.8 \mathrm{~Hz}, 3 \mathrm{H})$, $0.84(\mathrm{t}, J=7.2 \mathrm{~Hz}, 3 \mathrm{H}) ;{ }^{13} \mathrm{C}$ NMR $\left(\mathrm{d}_{6}\right.$-DMSO, $\left.100 \mathrm{MHz}\right): 159.6,147.3,127.1,123.6,120.5$, 114.8, 55.6, 49.4, 41.9, 29.3, 20.8, 11.3; HRMS: calc. for $\mathrm{C}_{14} \mathrm{H}_{20} \mathrm{~N}_{3} \mathrm{OS}(\mathrm{M}+\mathrm{H})^{+}, 278.1322$, found, 278.1326 .<smiles>COc1ccc(-c2cn(CSCc3ccccc3)nn2)cc1</smiles>

\section{3) 1-((Benzylthio)methyl)-4-(4-methoxyphenyl)-1H-1,2,3-triazole (6d)}

The reaction of $1 \mathbf{a}(39.6 \mathrm{mg}, 0.3 \mathrm{mmol})$, DPPA (165.0 mg, $0.6 \mathrm{mmol}), \mathrm{Cu}(\mathrm{acac})_{2}(7.6 \mathrm{mg}, 0.03$ 
mmol) in dry $\mathrm{CH}_{3} \mathrm{~S}(=\mathrm{O}) \mathrm{CH}_{2} \mathrm{Ph}(0.5 \mathrm{~mL})$ afforded $32.6 \mathrm{mg}(35 \%)$ of $\mathbf{6 d} ; \mathbf{6 d}$ : white solid; ${ }^{1} \mathrm{H}$ NMR (d d $\left.^{-D M S O}, 400 \mathrm{MHz}\right): 8.46$ (s, 1H), $7.80(\mathrm{~d}, J=8.8 \mathrm{~Hz}, 2 \mathrm{H}), 7.35-7.31(\mathrm{~m}, 5 \mathrm{H}), 7.02$ (d, $J=8.8$ $\mathrm{Hz}, 2 \mathrm{H}), 5.49$ (s, 2H), 3.88 (s, 2H), 3.80 (s, 3H); ${ }^{13} \mathrm{C}$ NMR (d $\mathrm{d}_{6}$-DMSO, $\left.100 \mathrm{MHz}\right): 159.6,147.3$, $137.8,129.6,129.0,127.7,127.1,123.6,120.5,114.8,55.6,50.1,35.5$; HRMS: calc. for $\mathrm{C}_{17} \mathrm{H}_{18} \mathrm{~N}_{3} \mathrm{OS}(\mathrm{M}+\mathrm{H})^{+}, 312.1165$, found, 312.1169 .<smiles>COc1ccc(-c2cn(CSSC)nn2)cc1</smiles>

\section{4) 4-(4-Methoxyphenyl)-1-((methyldisulfanyl)methyl)-1H-1,2,3-triazole (6e)}

The reaction of $1 \mathrm{a}(39.6 \mathrm{mg}, 0.3 \mathrm{mmol})$, DPPA (165.0 mg, $0.6 \mathrm{mmol}), \mathrm{Cu}(\mathrm{acac})_{2}(7.6 \mathrm{mg}, 0.03$ $\mathrm{mmol})$ in dry $\mathrm{CH}_{3} \mathrm{~S}(=\mathrm{O}) \mathrm{SCH}_{3}(0.5 \mathrm{~mL})$ afforded $48.9 \mathrm{mg}(61 \%)$ of $\mathbf{6 e} ; \mathbf{6 e}$ : yellow solid; ${ }^{1} \mathrm{H} \mathrm{NMR}$ (d6-DMSO, $400 \mathrm{MHz}): 8.56(\mathrm{~s}, 1 \mathrm{H}), 7.80(\mathrm{~d}, J=8.8 \mathrm{~Hz}, 2 \mathrm{H}), 7.02(\mathrm{~d}, J=8.8 \mathrm{~Hz}, 2 \mathrm{H}), 5.73(\mathrm{~s}$, $2 \mathrm{H}), 3.79$ (s, 3H), $2.22(\mathrm{~s}, 3 \mathrm{H}) ;{ }^{13} \mathrm{C}$ NMR (d 6 -DMSO, $\left.100 \mathrm{MHz}\right): 159.7,147.4,137.8,127.2$, 123.4, 120.9, 55.6, 55.5, 22.9; HRMS: calc. for $\mathrm{C}_{11} \mathrm{H}_{14} \mathrm{~N}_{3} \mathrm{OS}$ ( $(\mathrm{M}+\mathrm{H})^{+}, 268.0573$, found, 268.0578 .<smiles>C#CCCCOc1ccc2c(c1)CC[C@H]1[C@@H]2CC[C@]2(C)C(=O)CC[C@@H]12</smiles>

35)13-Methyl-3-(pent-4-yn-1-yloxy)-7,8,9,11,12,13,15,16-octahydro-6H-cyclopenta[a]phenant hren-17(14H)-one (7)

white solid; ${ }^{1} \mathrm{H}$ NMR $\left(\mathrm{CDCl}_{3}, 400 \mathrm{MHz}\right): 7.19$ (d, $\left.J=8.4 \mathrm{~Hz}, 1 \mathrm{H}\right), 6.72(\mathrm{~d}, J=8.4 \mathrm{~Hz}, 1 \mathrm{H}), 6.66$ (s, 1H), $4.04(\mathrm{t}, J=6.2 \mathrm{~Hz}, 2 \mathrm{H}), 2.89(\mathrm{~d}, J=5.2 \mathrm{~Hz}, 2 \mathrm{H}), 2.54-2.46(\mathrm{~m}, 1 \mathrm{H}), 2.40-2.38(\mathrm{~m}, 3 \mathrm{H})$, $2.25(\mathrm{~s}, 1 \mathrm{H}), 2.18-2.07(\mathrm{~m}, 1 \mathrm{H}), 2.02-1.96(\mathrm{~m}, 6 \mathrm{H}), 1.65-1.40(\mathrm{~m}, 6 \mathrm{H}), 0.91(\mathrm{~s}, 3 \mathrm{H}) ;{ }^{13} \mathrm{C} \mathrm{NMR}$ $\left(\mathrm{CDCl}_{3}, 100 \mathrm{MHz}\right): 220.7,156.8,137.6,132.0,126.2,114.4,112.0,83.4,68.7,65.9,50.3,47.8$, 43.8, 38.2, 35.7, 31.5, 29.5, 28.1, 26.4, 25.8, 21.4, 15.0, 13.7; HRMS: calc. for $\mathrm{C}_{23} \mathrm{H}_{29} \mathrm{O}_{2}(\mathrm{M}+\mathrm{H})^{+}$, 337.2162, found, 337.2167 . 
<smiles>CSCn1cc(CCCOc2ccc3c(c2)CC[C@H]2[C@@H]4CCC(=O)[C@@]4(C)CC[C@@H]32)nn1</smiles>

36)13-Methyl-3-(3-(1-((methylthio)methyl)-1H-1,2,3-triazol-4-yl)propoxy)-7,8,9,11,12,13,15,1 6-octahydro-6H-cyclopenta[a]phenanthren-17(14H)-one (8)

The reaction of $5(100.8 \mathrm{mg}, 0.3 \mathrm{mmol}), \mathrm{Cu}(\mathrm{acac})_{2}(7.8 \mathrm{mg}, 0.03 \mathrm{mmol})$ in dry DMSO $(2 \mathrm{~mL})$ afforded $94.8 \mathrm{mg}(72 \%)$ of $\mathbf{6}$; the reaction of 7 (102.0 mg, $0.3 \mathrm{mmol}), \mathrm{Cu}(\mathrm{acac})_{2}(7.8 \mathrm{mg}, 0.03$ mmol) in dry DMSO $(2 \mathrm{~mL})$ afforded $79.2 \mathrm{mg}(60 \%)$ of 6; 6: yellow solid; ${ }^{1} \mathrm{H} \mathrm{NMR}\left(\mathrm{CDCl}_{3}, 400\right.$ MHz): $7.53(\mathrm{~s}, 1 \mathrm{H}), 7.19$ (d, $J=8.8 \mathrm{~Hz}, 1 \mathrm{H}), 6.70(\mathrm{~d}, J=6.0 \mathrm{~Hz}, 1 \mathrm{H}), 6.64(\mathrm{~s}, 1 \mathrm{H}), 5.29(\mathrm{~s}, 2 \mathrm{H})$, $4.00(\mathrm{t}, J=5.6 \mathrm{~Hz}, 2 \mathrm{H}), 2.94-2.88(\mathrm{~m}, 4 \mathrm{H}), 2.53-2.46(\mathrm{~m}, 1 \mathrm{H}), 2.40-2.37(\mathrm{~m}, 1 \mathrm{H}), 2.24-2.14(\mathrm{~m}$, $4 \mathrm{H}), 2.11(\mathrm{~s}, 3 \mathrm{H}), 2.05-1.93(\mathrm{~m} \mathrm{3H}), 1.67-1.40(\mathrm{~m}, 6 \mathrm{H}), 0.90(\mathrm{~s}, 3 \mathrm{H}) ;{ }^{13} \mathrm{C} \mathrm{NMR}\left(\mathrm{CDCl}_{3}, 100\right.$ MHz): 220.8, 156.8, 148.2, 137.7, 132.0, 126.2, 120.2, 114.4, 112.1, 66.6, 52.3, 50.3, 47.9, 43.9, $38.3,35.8,31.5,29.6,28.8,26.4,25.8,22.2,21.5,14.7,13.8$; HRMS: calc. for $\mathrm{C}_{25} \mathrm{H}_{34} \mathrm{~N}_{3} \mathrm{O}_{2} \mathrm{~S}$ $(\mathrm{M}+\mathrm{H})^{+}, 440.2366$, found, 440.2358 .<smiles>C#CCCCOc1ccc2c(c1)CC[C@H]1[C@@H]2CC[C@]2(C)C(O)CC[C@@H]12</smiles>

37)13-Methyl-3-(pent-4-yn-1-yloxy)-7,8,9,11,12,13,14,15,16,17-decahydro-6H-cyclopenta[a]p henanthren-17-ol (9)

white solid; ${ }^{1} \mathrm{H}$ NMR $\left(\mathrm{CDCl}_{3}, 400 \mathrm{MHz}\right): 7.18$ (d, $\left.J=8.4 \mathrm{~Hz}, 1 \mathrm{H}\right) ; 6.69$ (d, $\left.J=8.4 \mathrm{~Hz}, 1 \mathrm{H}\right) ; 6.63$ (s, 1H); $4.02(\mathrm{t}, J=6.0 \mathrm{H}, 2 \mathrm{H}), 3.70(\mathrm{t}, J=8.2 \mathrm{~Hz}, 1 \mathrm{H}), 2.86-2.76(\mathrm{~m}, 2 \mathrm{H}), 2.40-2.36(\mathrm{~m}, 2 \mathrm{H})$, 2.31-2.27 (m, 1H), 2.19-2.07 (m, 2H), 2.05-1.92 (m, 4H), 1.88-1.84 (m, 1H), 1.78-1.64 (m, 2H), $1.52-1.29(\mathrm{~m}, 7 \mathrm{H}), 0.76(\mathrm{~s}, 3 \mathrm{H}) .{ }^{13} \mathrm{C} \mathrm{NMR}\left(\mathrm{CDCl}_{3}, 100 \mathrm{MHz}\right): 156.6,137.8,132.6,126.2,114.4$, 111.9, 83.5, 81.7, 68.7, 65.9, 49.9, 43.8, 43.1, 38.7, 36.4, 30.4, 29.7, 28.1, 27.1, 26.2, 23.0, 15.1, 11.0. HRMS: calc. for $\mathrm{C}_{23} \mathrm{H}_{31} \mathrm{O}_{2}(\mathrm{M}+\mathrm{H})^{+}, 339.2319$, found, 339.2321 .<smiles>COc1ccc(-c2cn(CS(C)(=O)=O)nn2)cc1</smiles> 
38) 4-(4-Methoxyphenyl)-1-((methylsulfinyl)methyl)-1H-1,2,3-triazole (10)

The reaction of $2 \mathbf{a}(72 \mathrm{mg}, 0.3 \mathrm{mmol})$, oxone $(0.45 \mathrm{mmol}, 270 \mathrm{mg})$, in $\mathrm{H}_{2} \mathrm{O}(3 \mathrm{~mL})$ was stirred at room temperature for $8 \mathrm{~h}$ afforded $61.9 \mathrm{mg}(77 \%)$ of 8 ; 8: white solid; ${ }^{1} \mathrm{H}$ NMR ( $\mathrm{d}_{6}$-DMSO, 400 MHz): 8.58 (s, 1H), 7.84 (dd, $J=6.8 \mathrm{~Hz}, J=2.0 \mathrm{~Hz}, 2 \mathrm{H}), 7.04$ (dd, $J=6.8 \mathrm{~Hz}, J=2.0 \mathrm{~Hz}, 2 \mathrm{H}$ ), 6.13 (s, 2H), 3.80 (s, 3H), 3.14 (s, 3H); ${ }^{13} \mathrm{C}$ NMR (d ${ }_{6}$-DMSO, $\left.100 \mathrm{MHz}\right): 159.8,147.2,127.3$, 123.0, 122.7, 114.9, 66.6, 55.7, 39.6; HRMS: calc. for $\mathrm{C}_{11} \mathrm{H}_{14} \mathrm{~N}_{3} \mathrm{O}_{3} \mathrm{~S}(\mathrm{M}+\mathrm{H})^{+}, 268.0750$, found, 268.0744.<smiles>COc1ccc(-c2cn(CS(C)=O)nn2)cc1</smiles>

11

39) 4-(4-Methoxyphenyl)-1-((methylsulfonyl)methyl)-1H-1,2,3-triazole (11)

The reaction of $2 \mathbf{a}(47.2 \mathrm{mg}, 0.2 \mathrm{mmol}), \mathrm{H}_{2} \mathrm{O}_{2}(30 \%$ aq., $40 \mu \mathrm{L}), \mathrm{HNO}_{3} 14 \mu \mathrm{L}$, in EtOH $(1 \mathrm{~mL})$ was stirred at room temperature for $4 \mathrm{~h}$, afforded $38.4 \mathrm{mg}$ (72\%) of 9; 9: white solid; ${ }^{1} \mathrm{H}$ NMR (d d $\left.^{-D M S O}, 400 \mathrm{MHz}\right): 8.49$ (s, 1H), 7.83 (d, $\left.J=8.4 \mathrm{~Hz}, 2 \mathrm{H}\right), 7.03$ (d, $\left.J=8.4 \mathrm{~Hz}, 2 \mathrm{H}\right), 5.84$ (d, $J=$ $13.6 \mathrm{~Hz}, 1 \mathrm{H}), 5.62(\mathrm{~d}, J=13.6 \mathrm{~Hz}, 1 \mathrm{H}), 3.79(\mathrm{~s}, 3 \mathrm{H}), 2.60(\mathrm{~s}, 3 \mathrm{H}) ;{ }^{13} \mathrm{C}$ NMR $\left(\mathrm{d}_{6}\right.$-DMSO, 100 MHz): 159.7, 146.9, 127.2, 123.3, 122.6, 114.9, 66.6, 55.7, 36.3; HRMS: calc. for $\mathrm{C}_{11} \mathrm{H}_{13} \mathrm{~N}_{3} \mathrm{NaO}_{2} \mathrm{~S}(\mathrm{M}+\mathrm{H})^{+}, 274.0621$, found, 274.0615. 

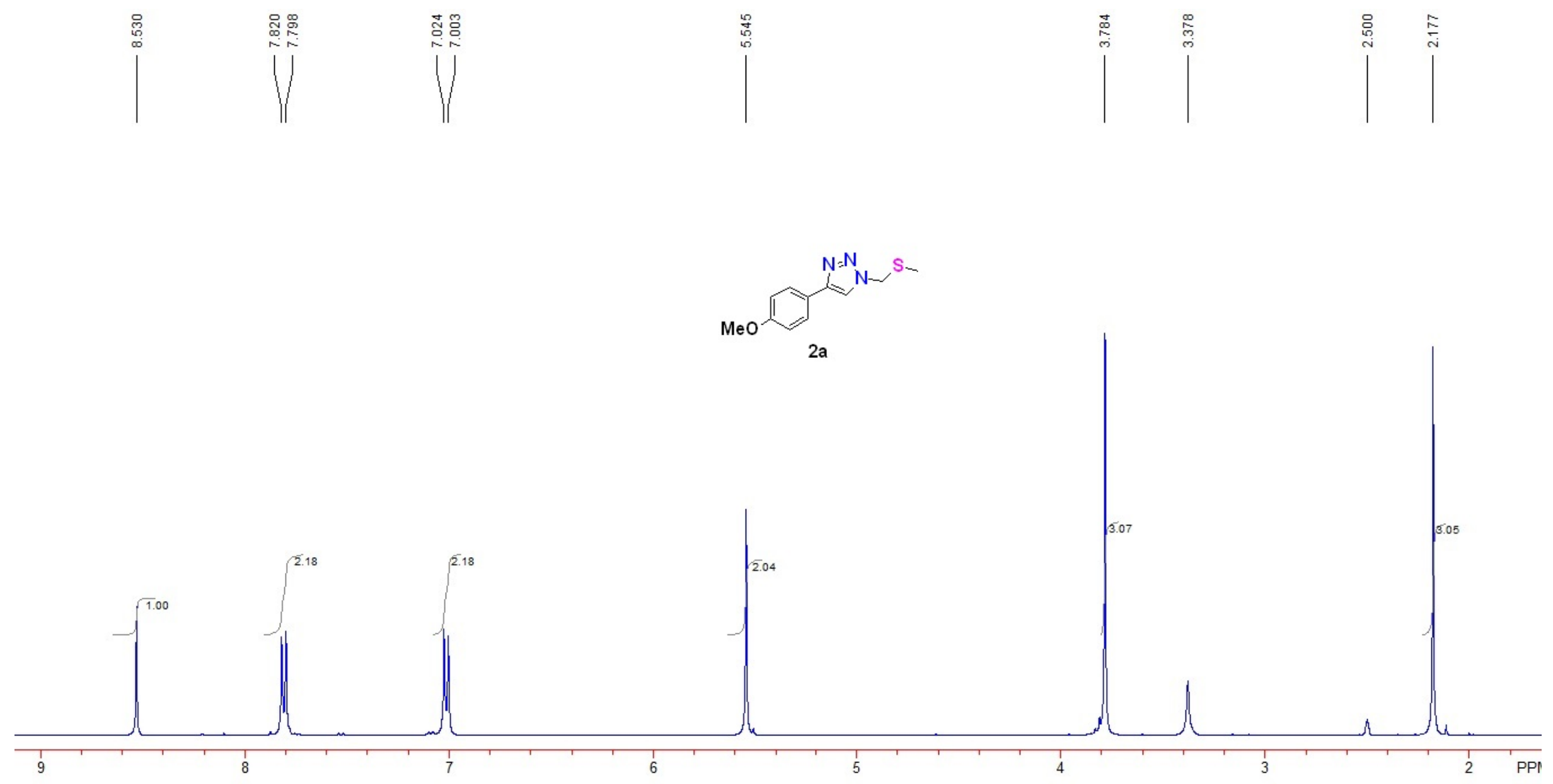


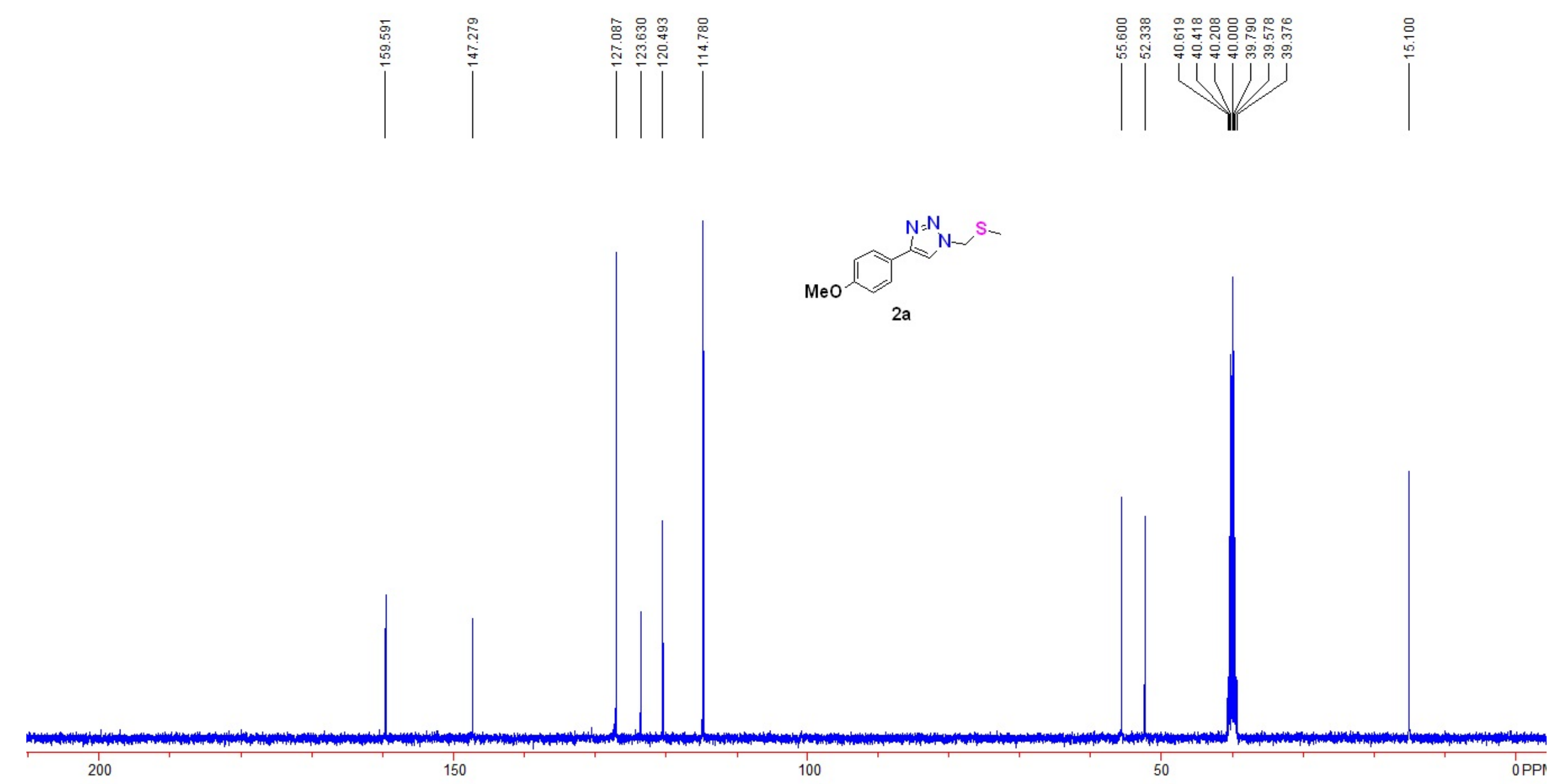



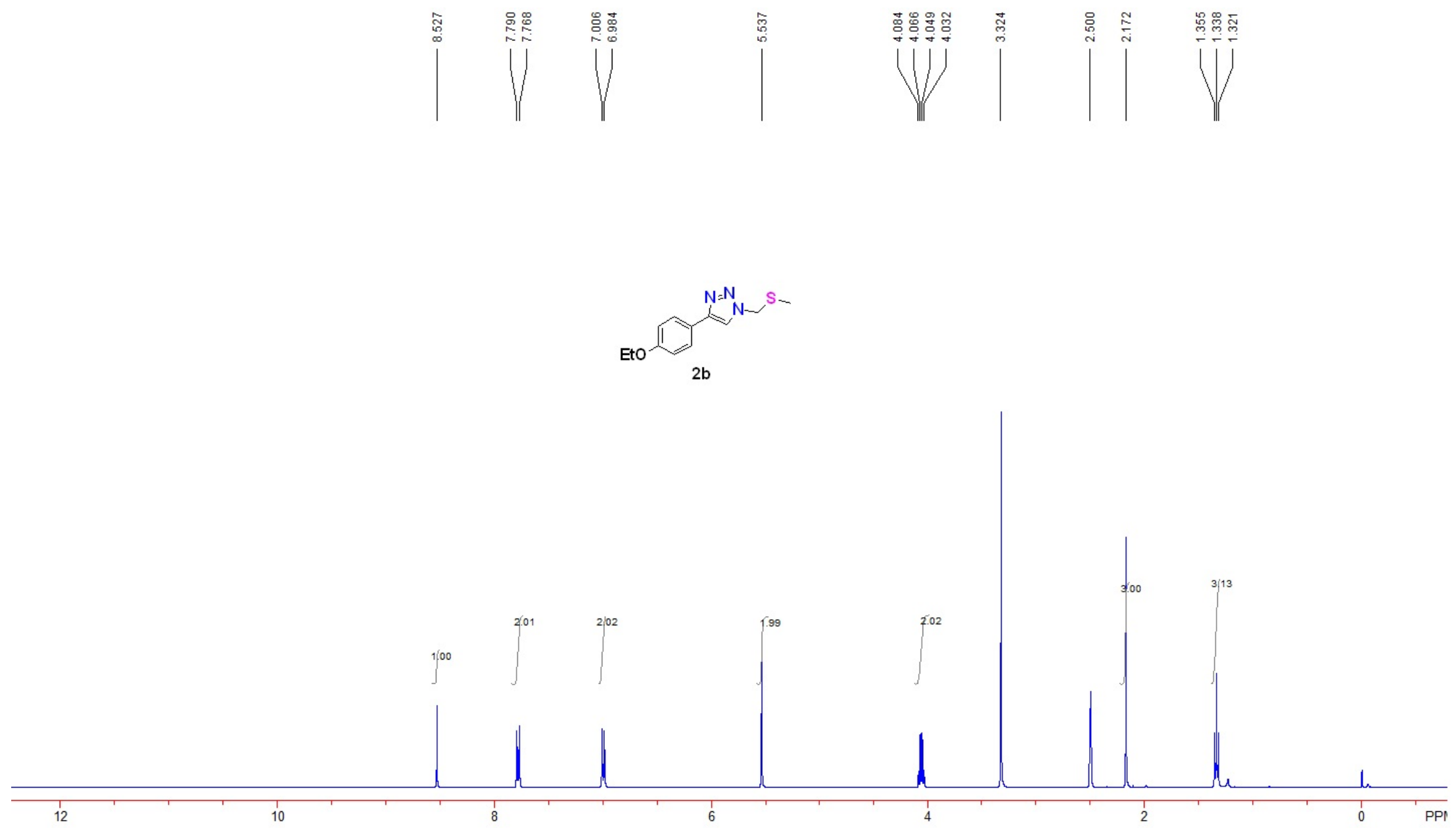

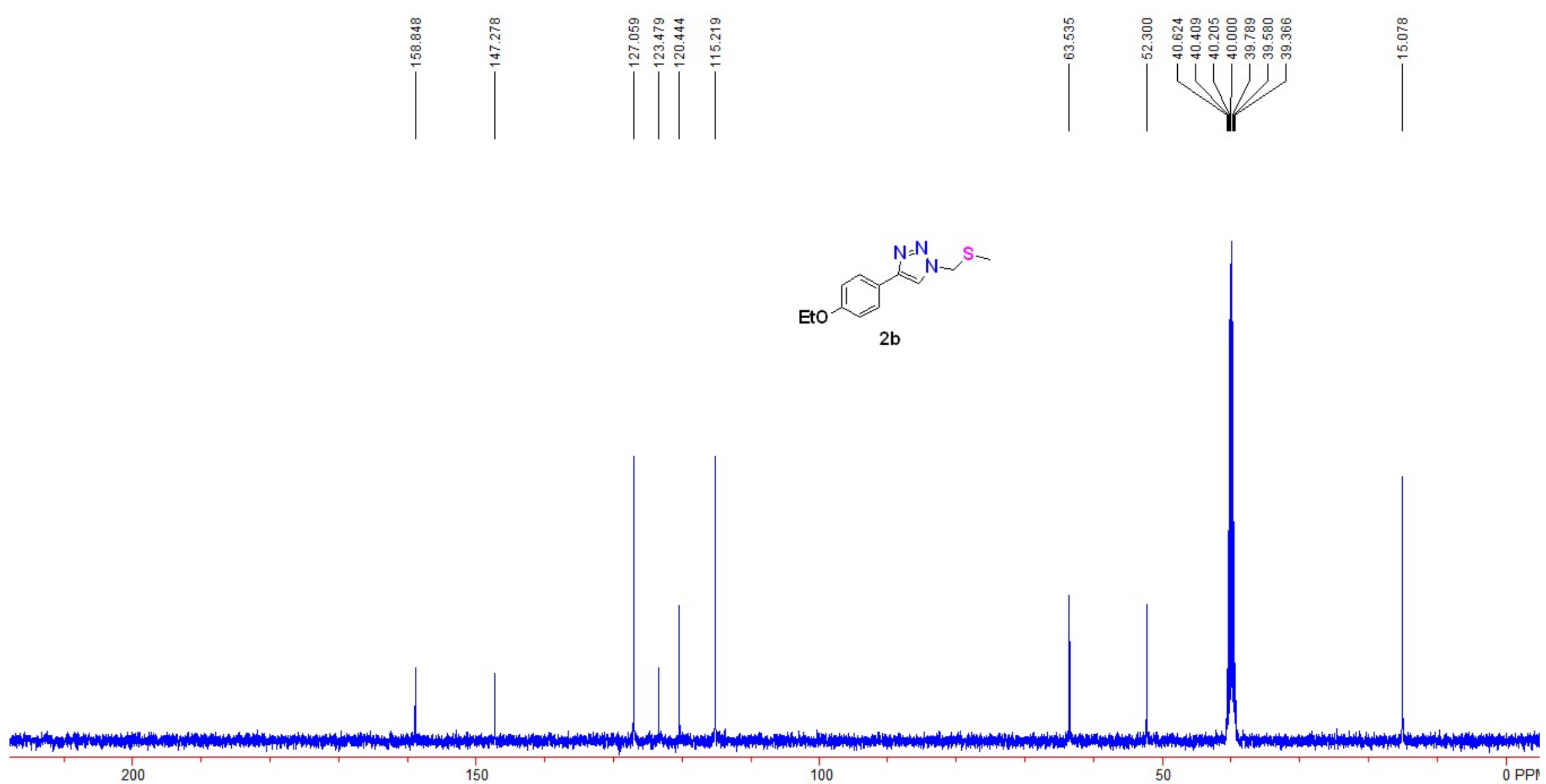

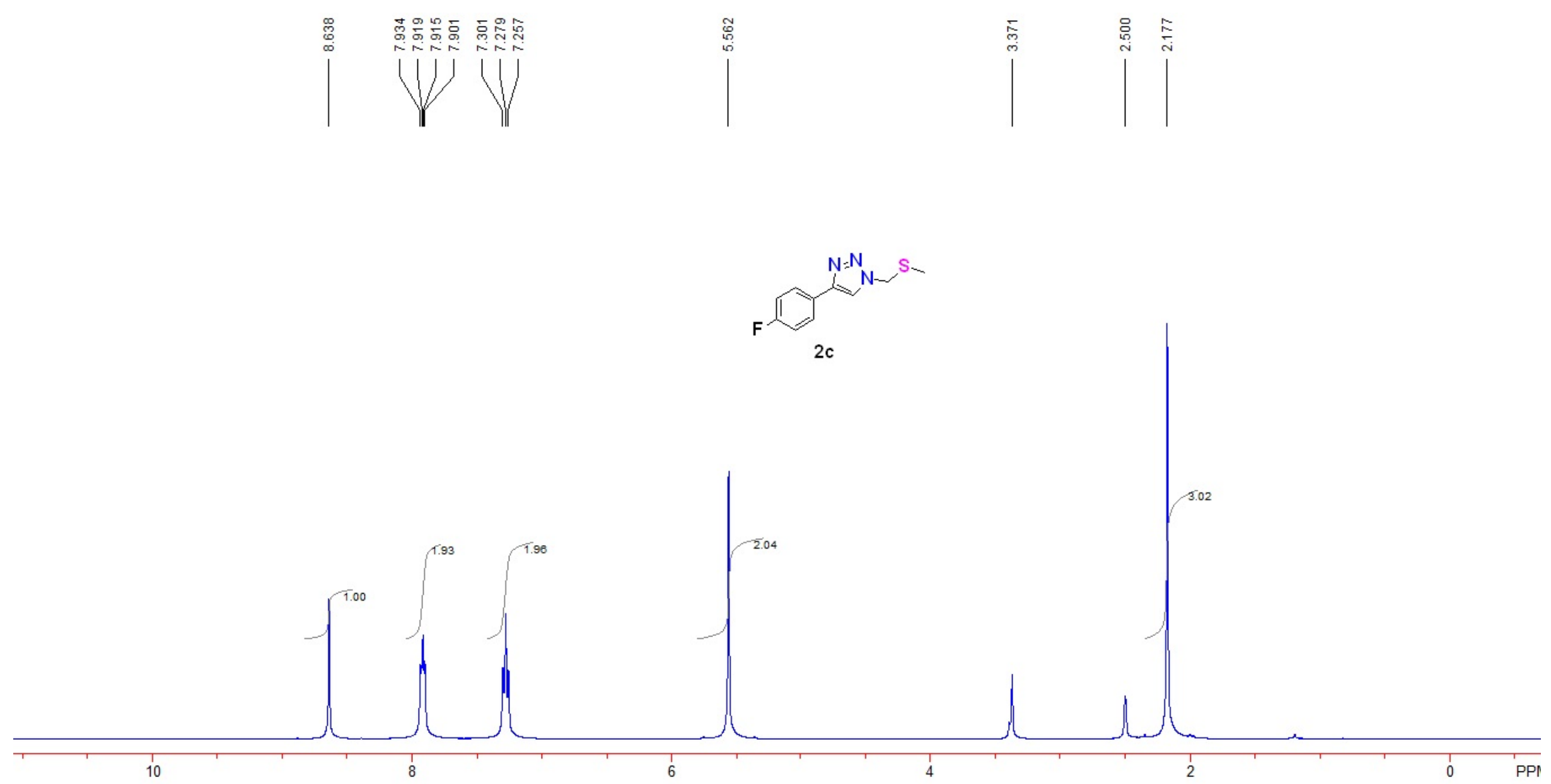


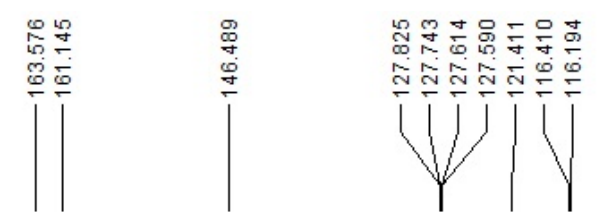

|
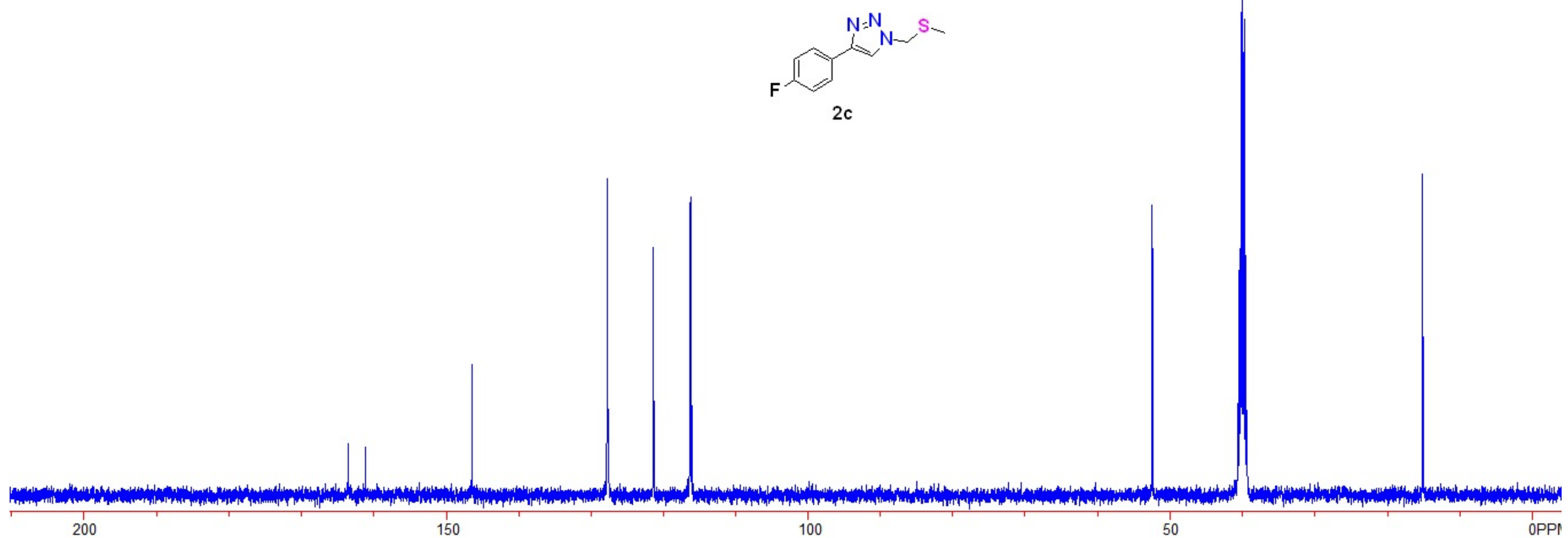

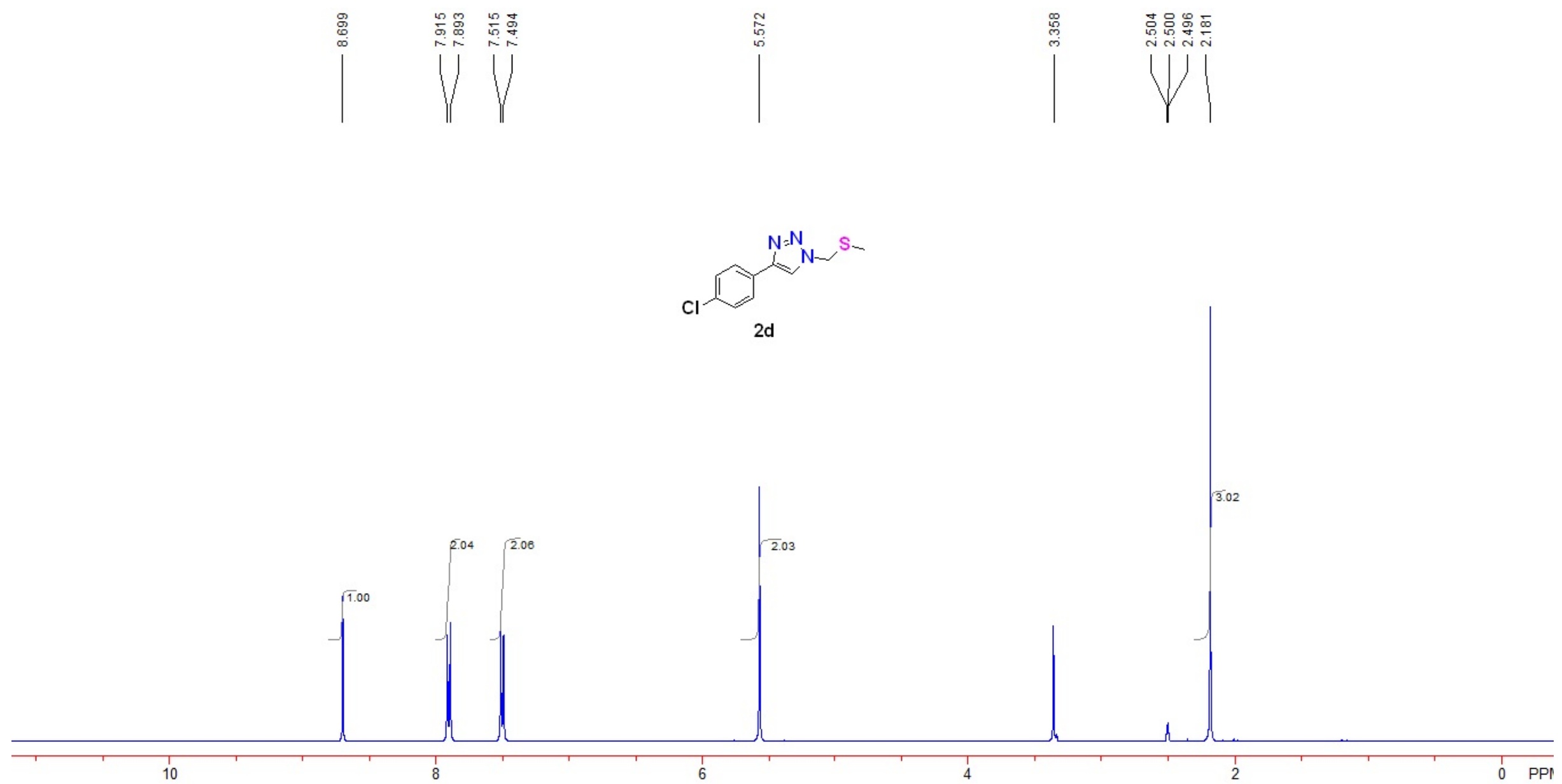

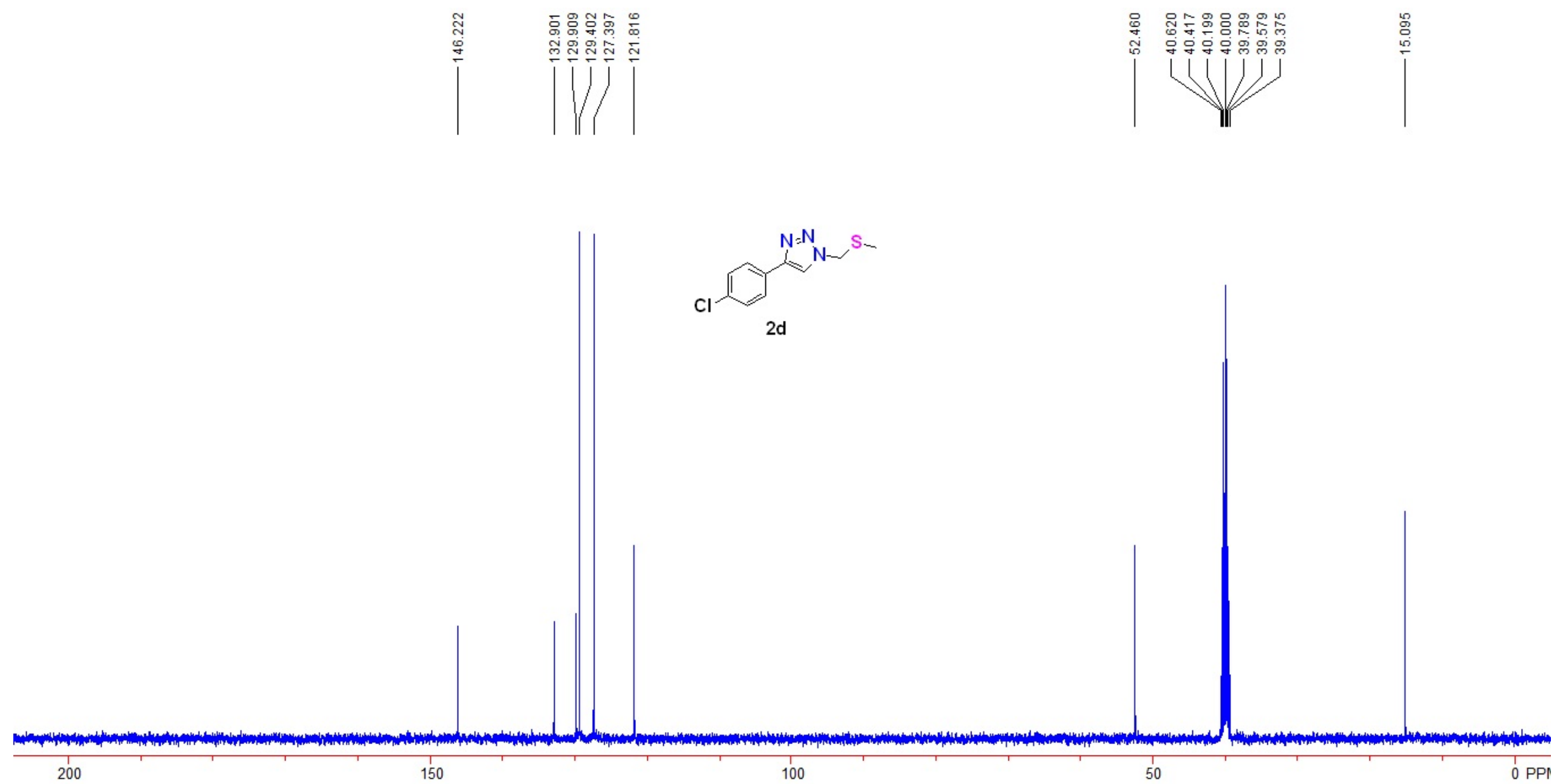


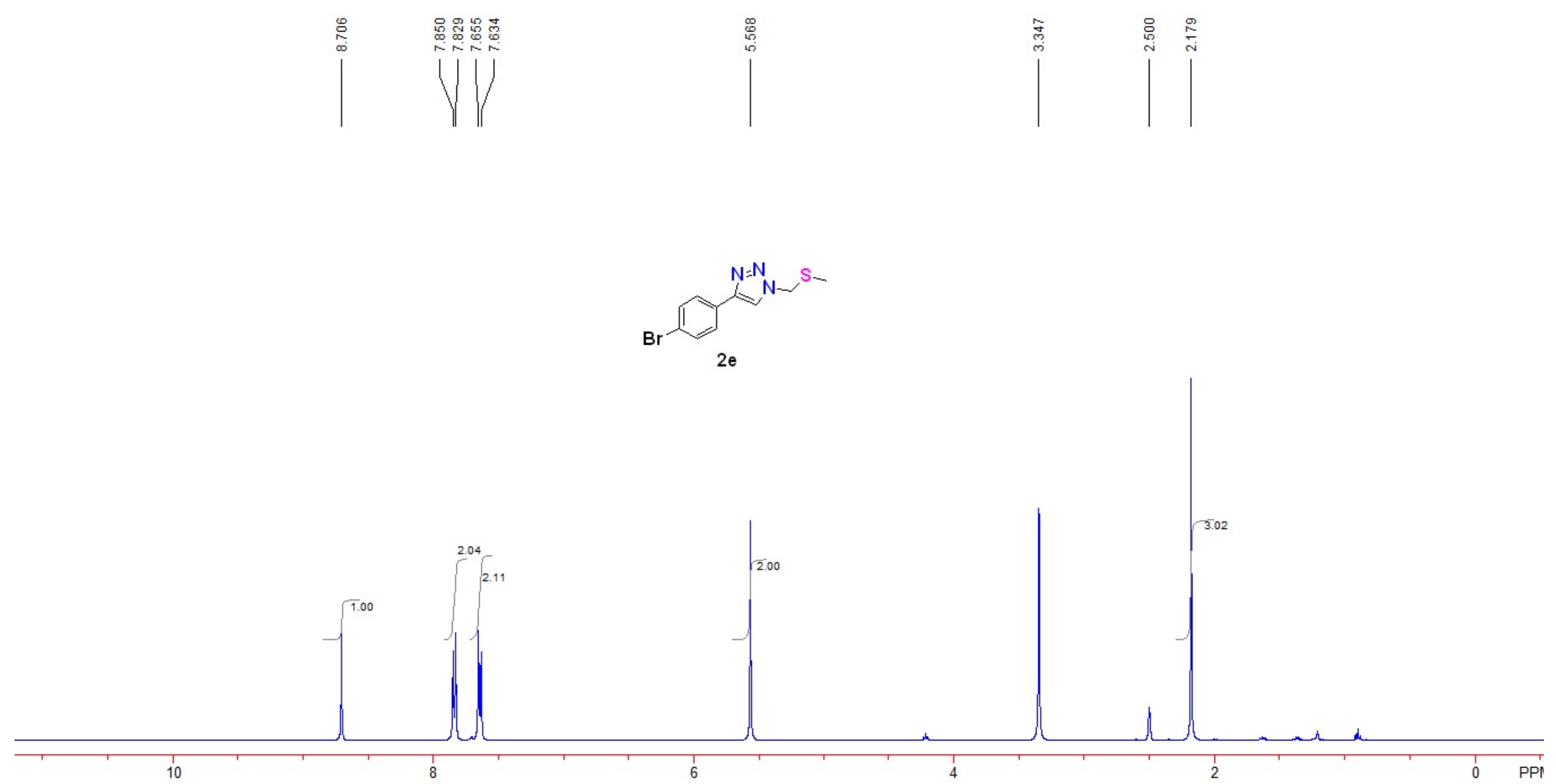



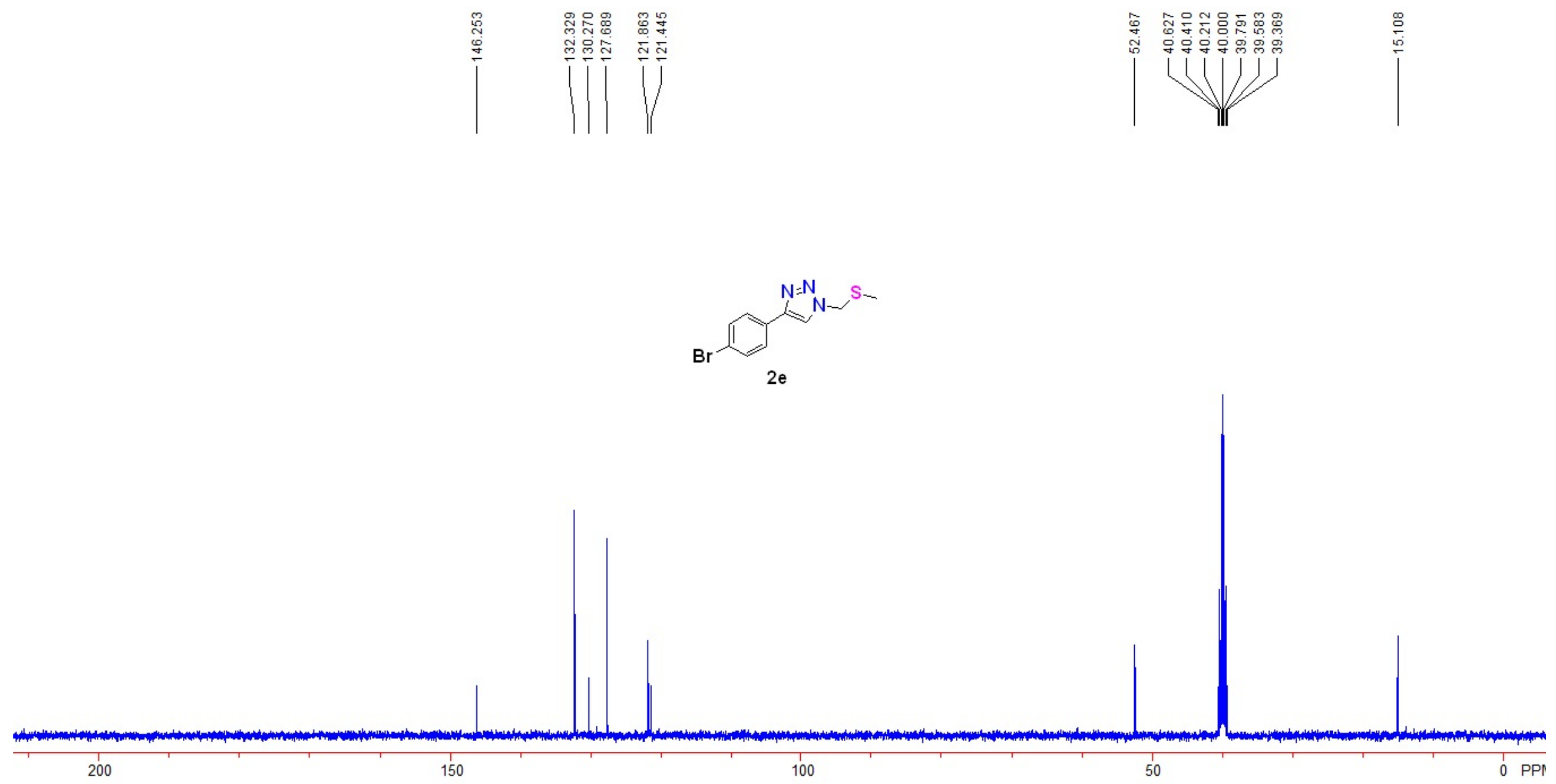

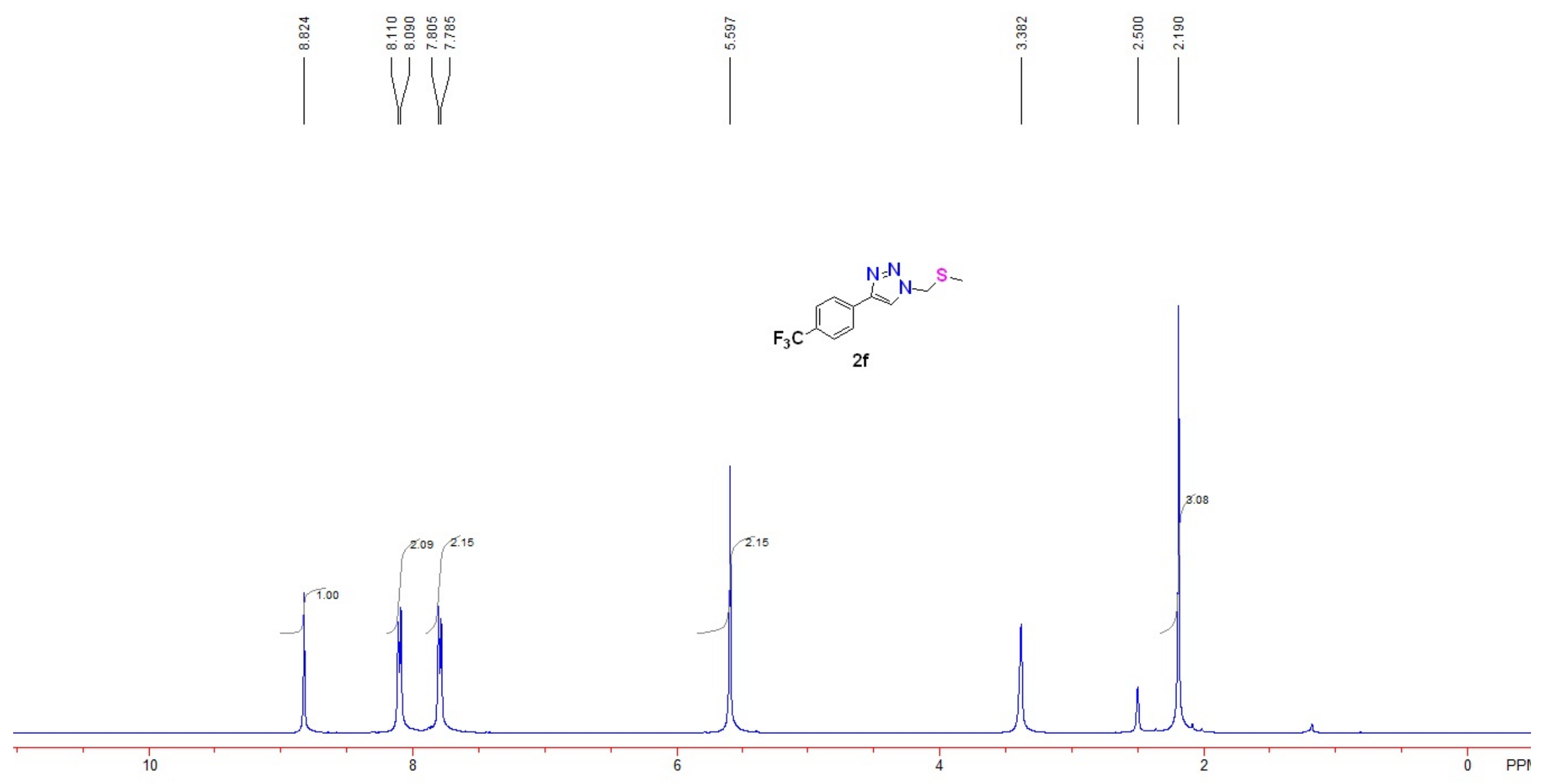

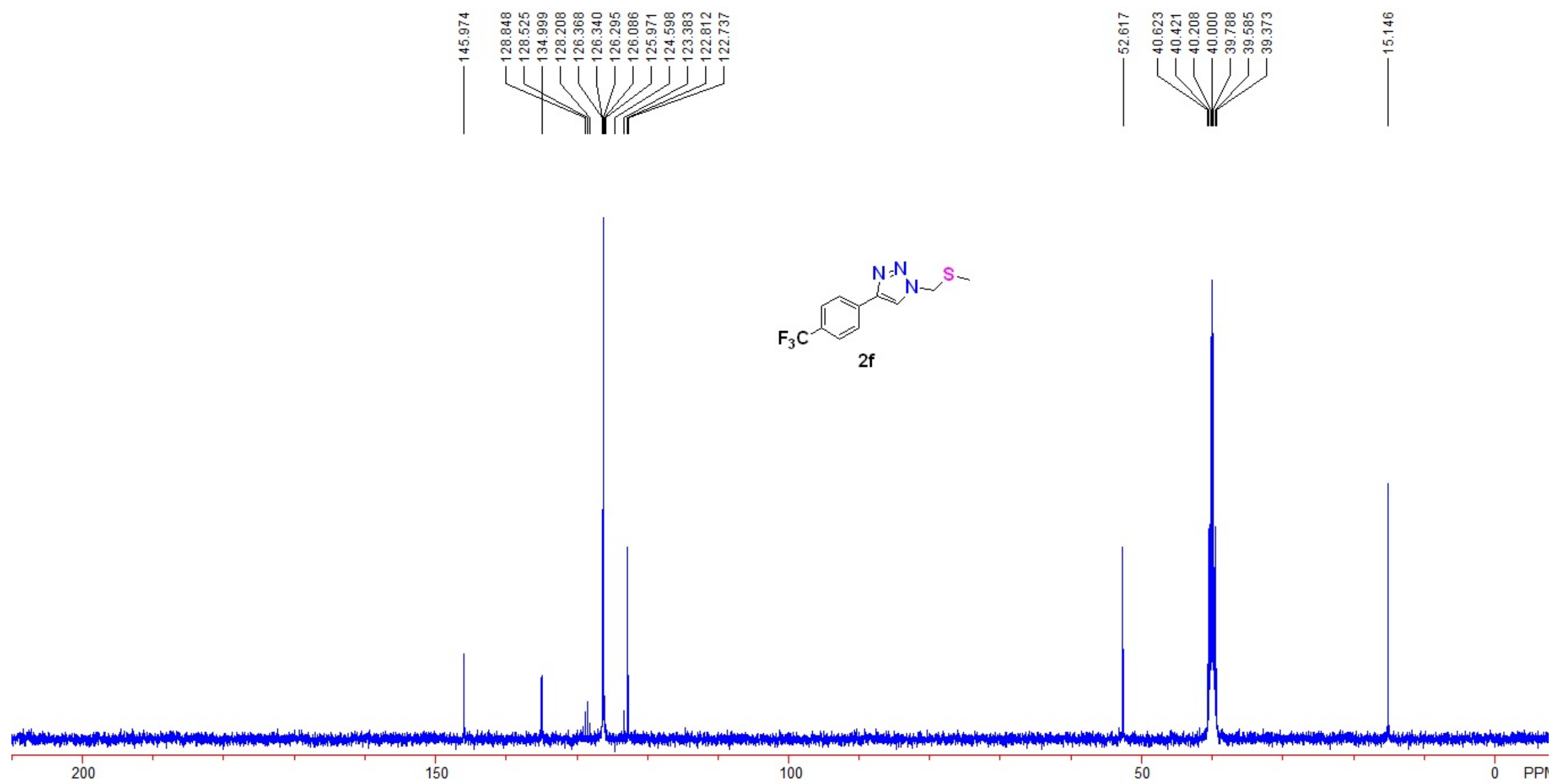

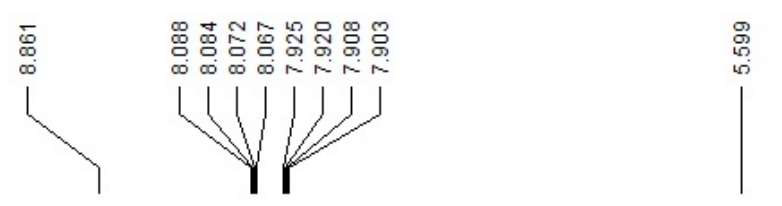

홍응

Y

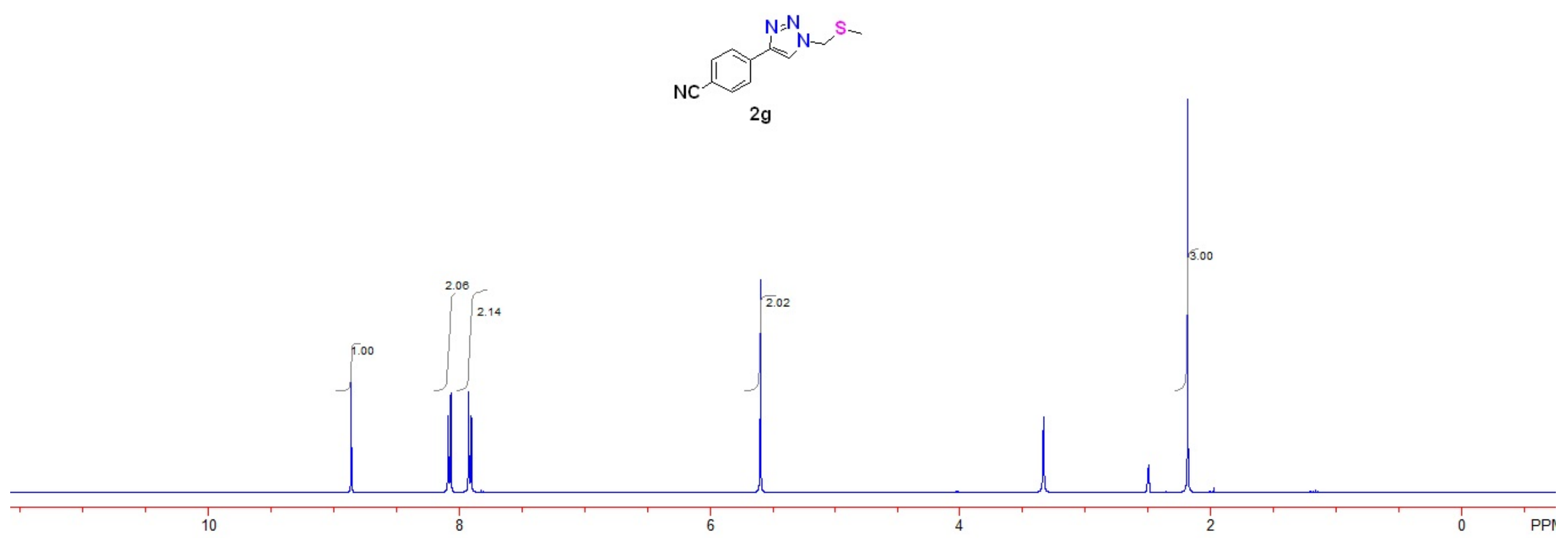



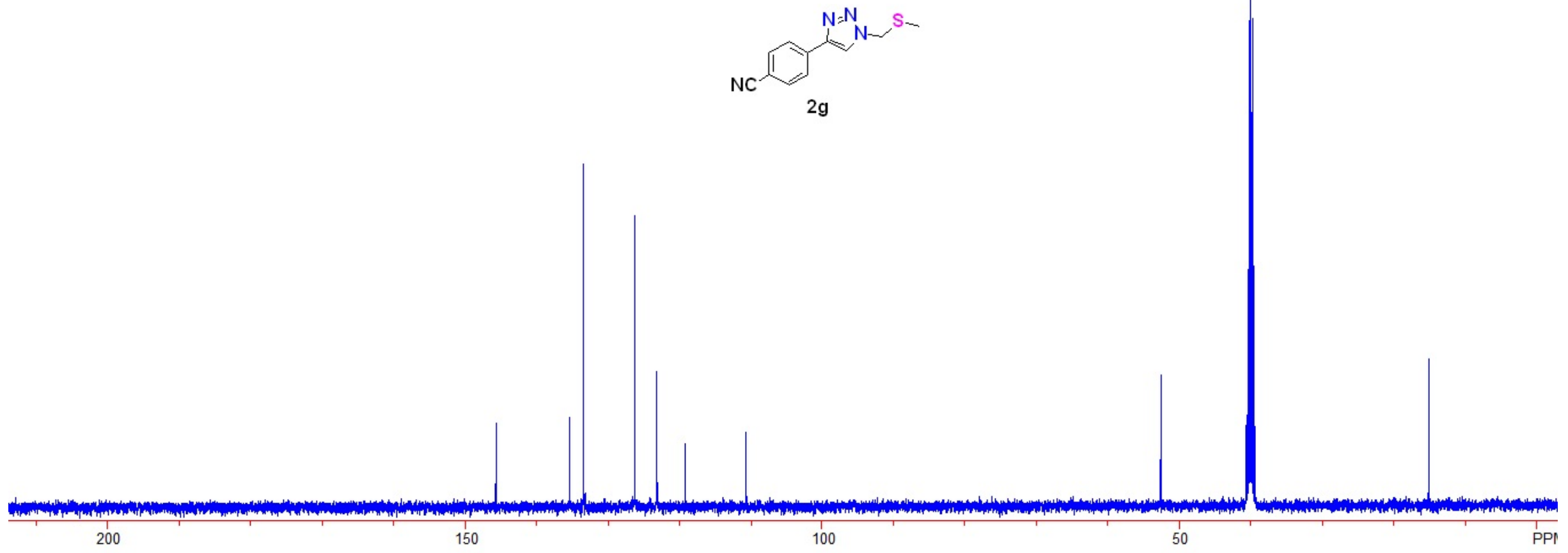

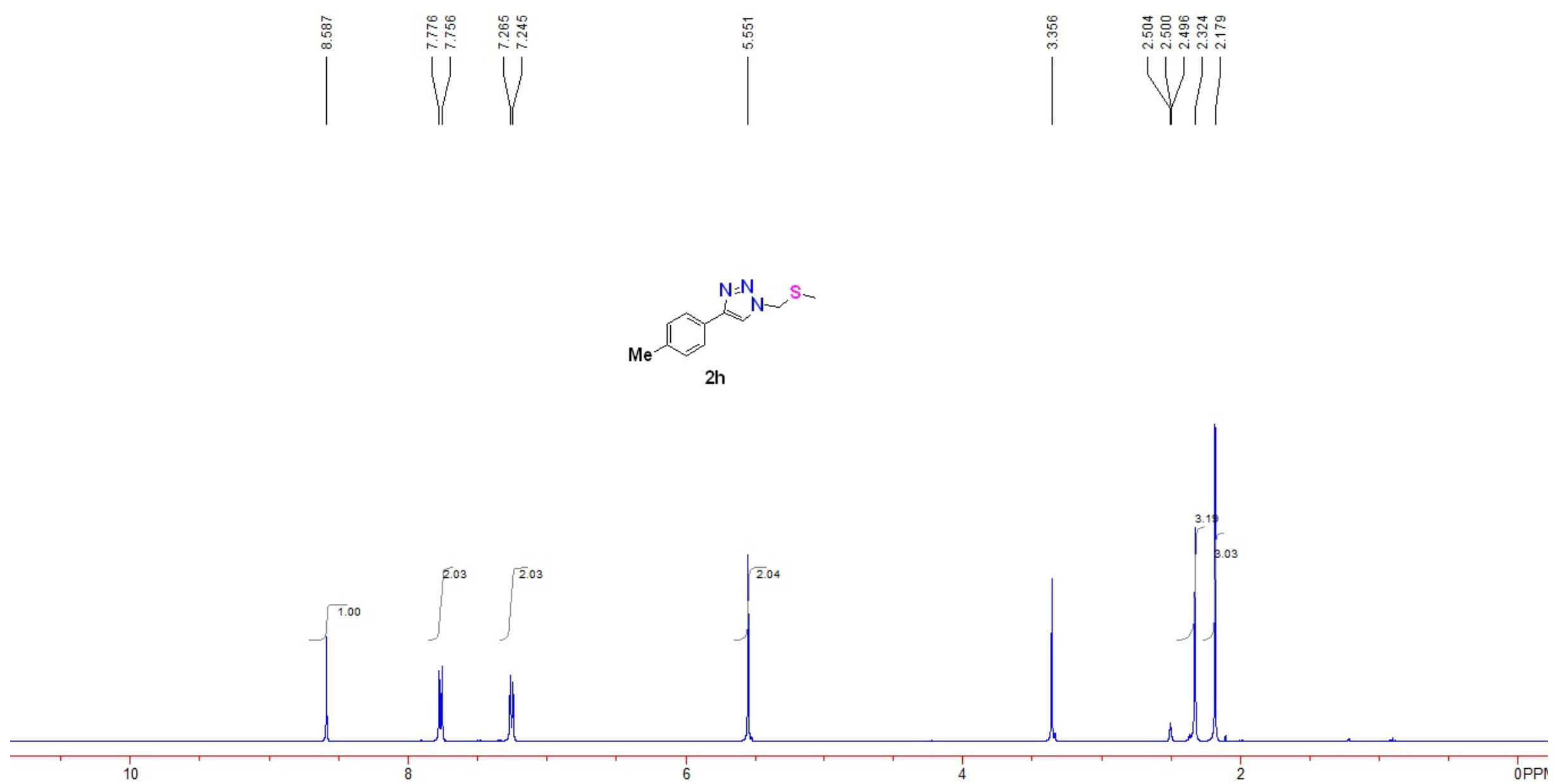

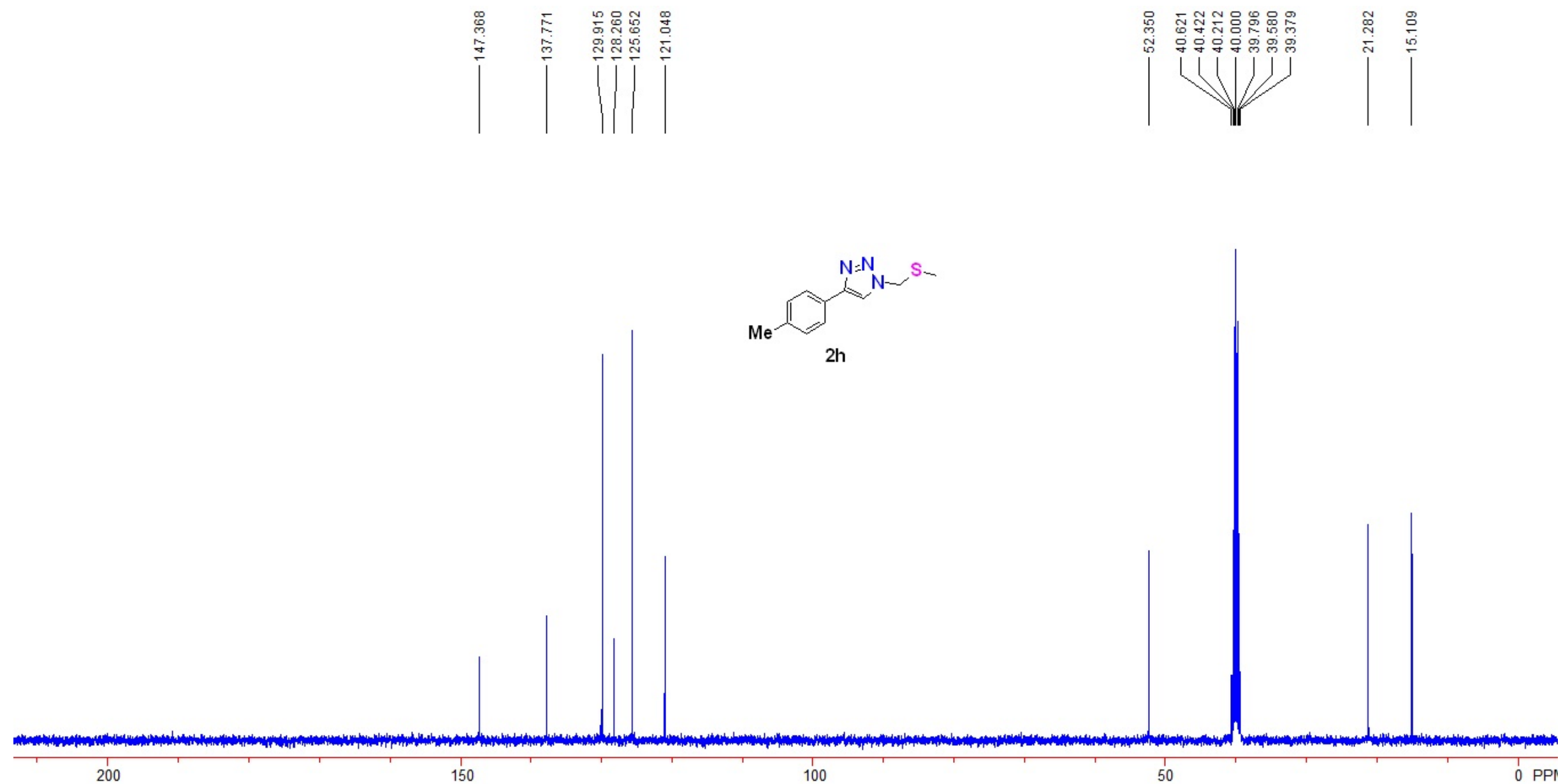


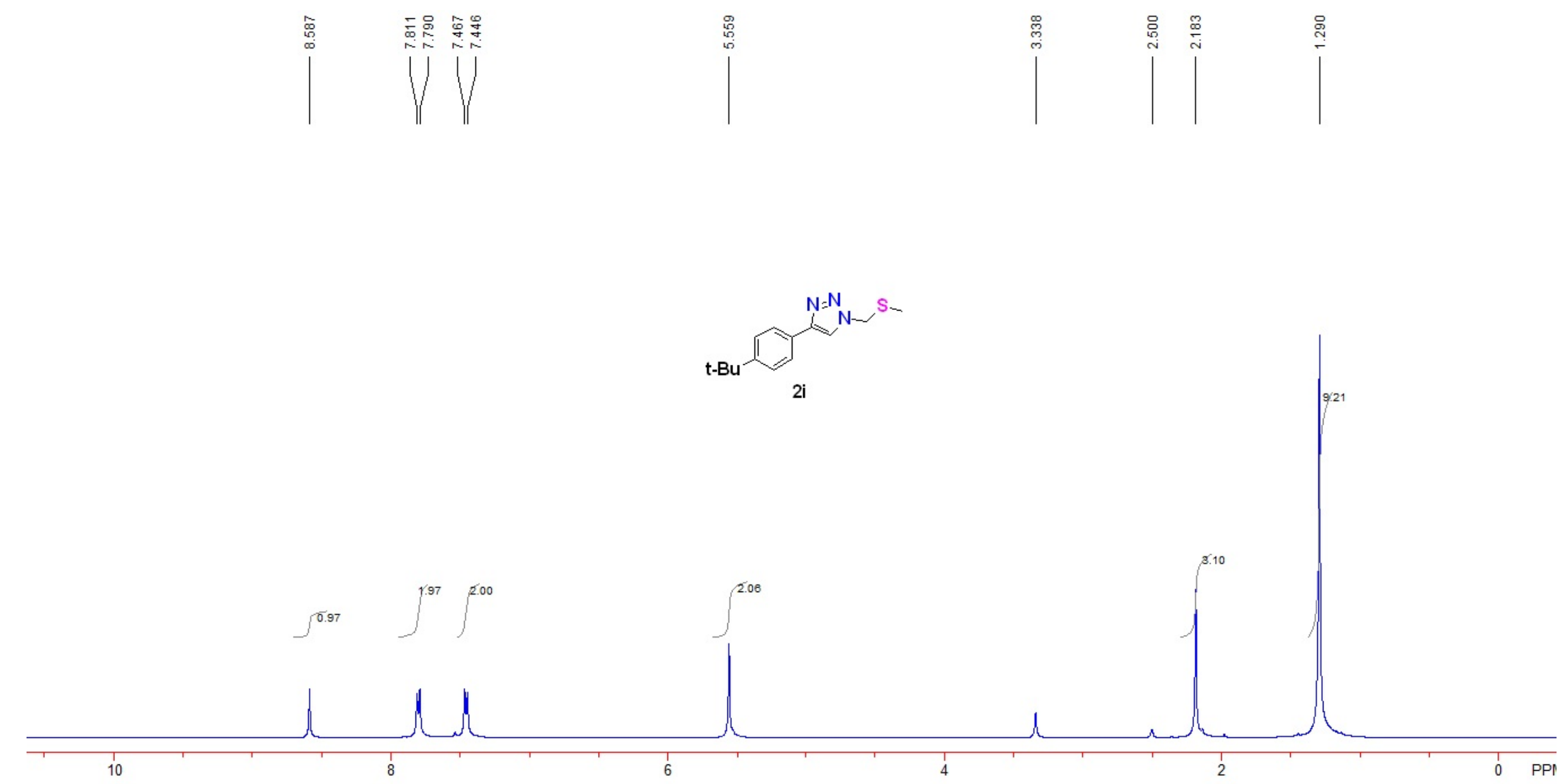




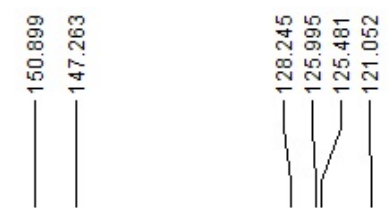

|
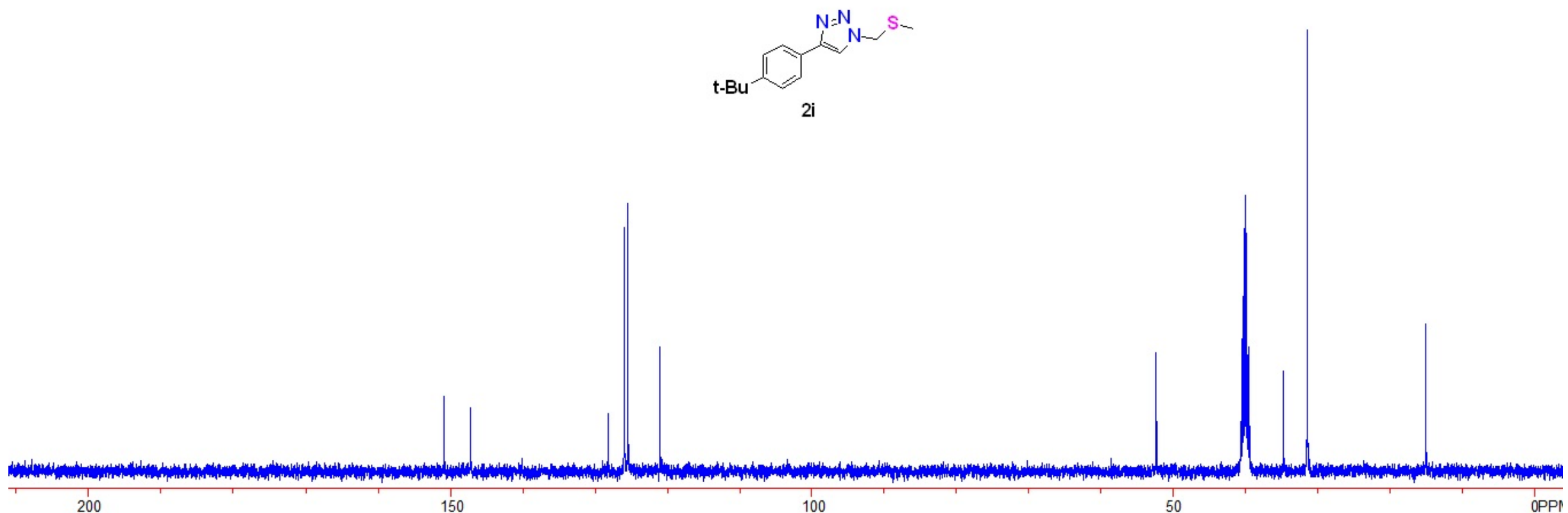

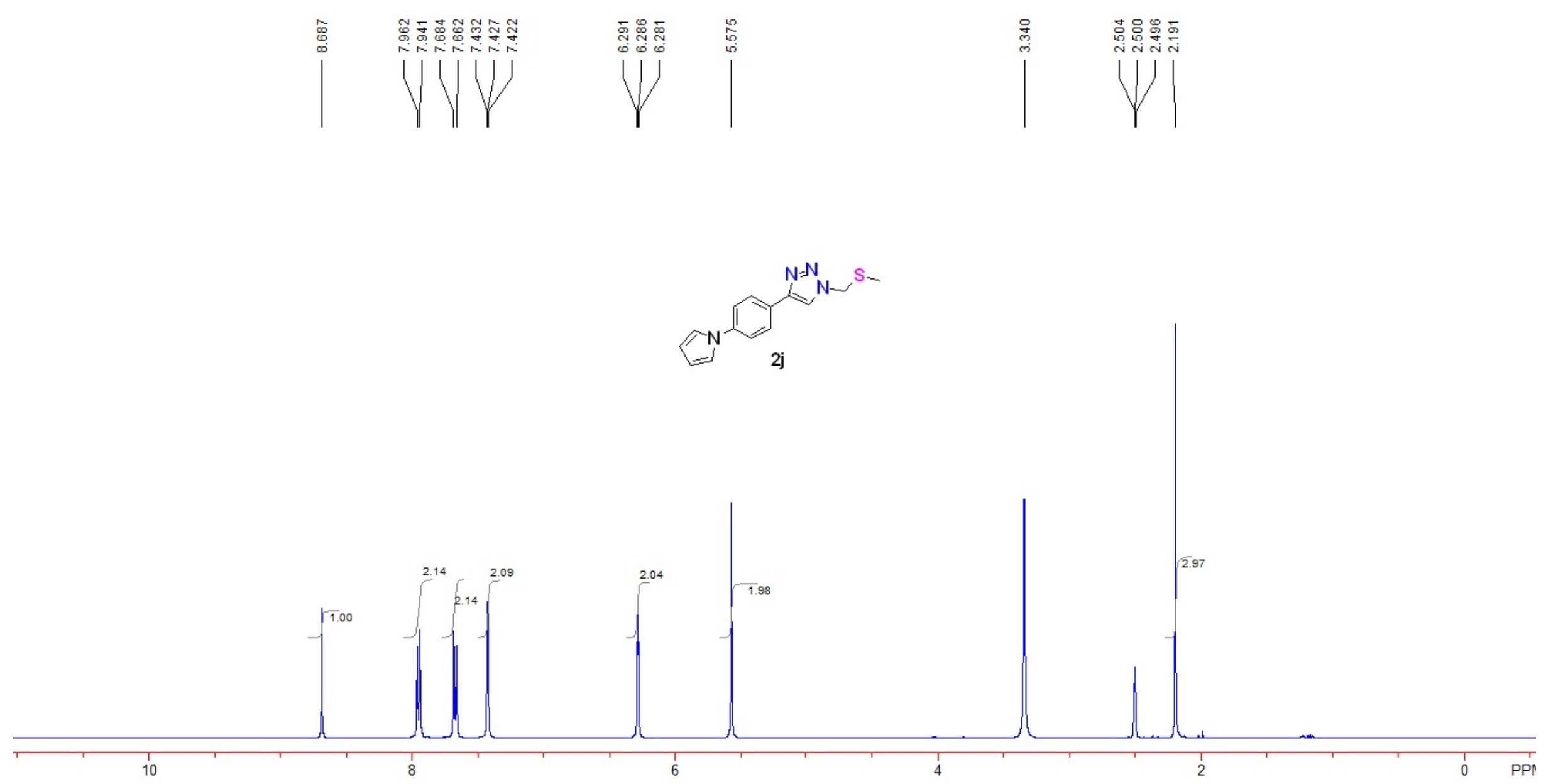


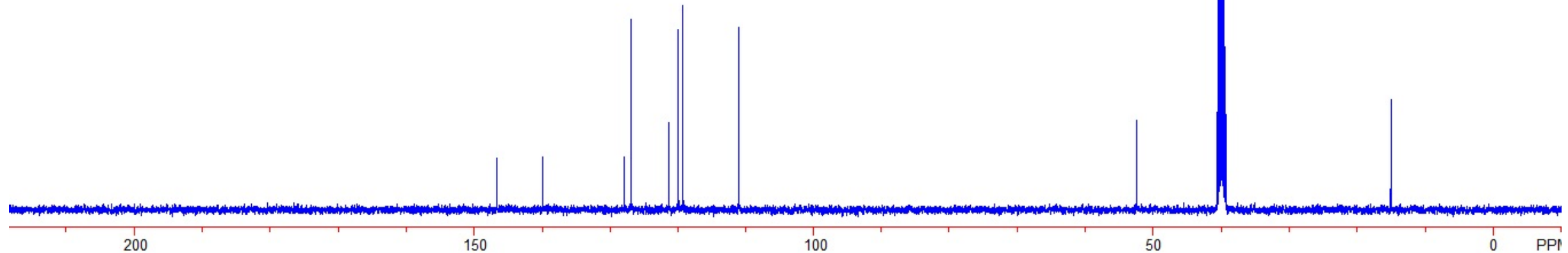




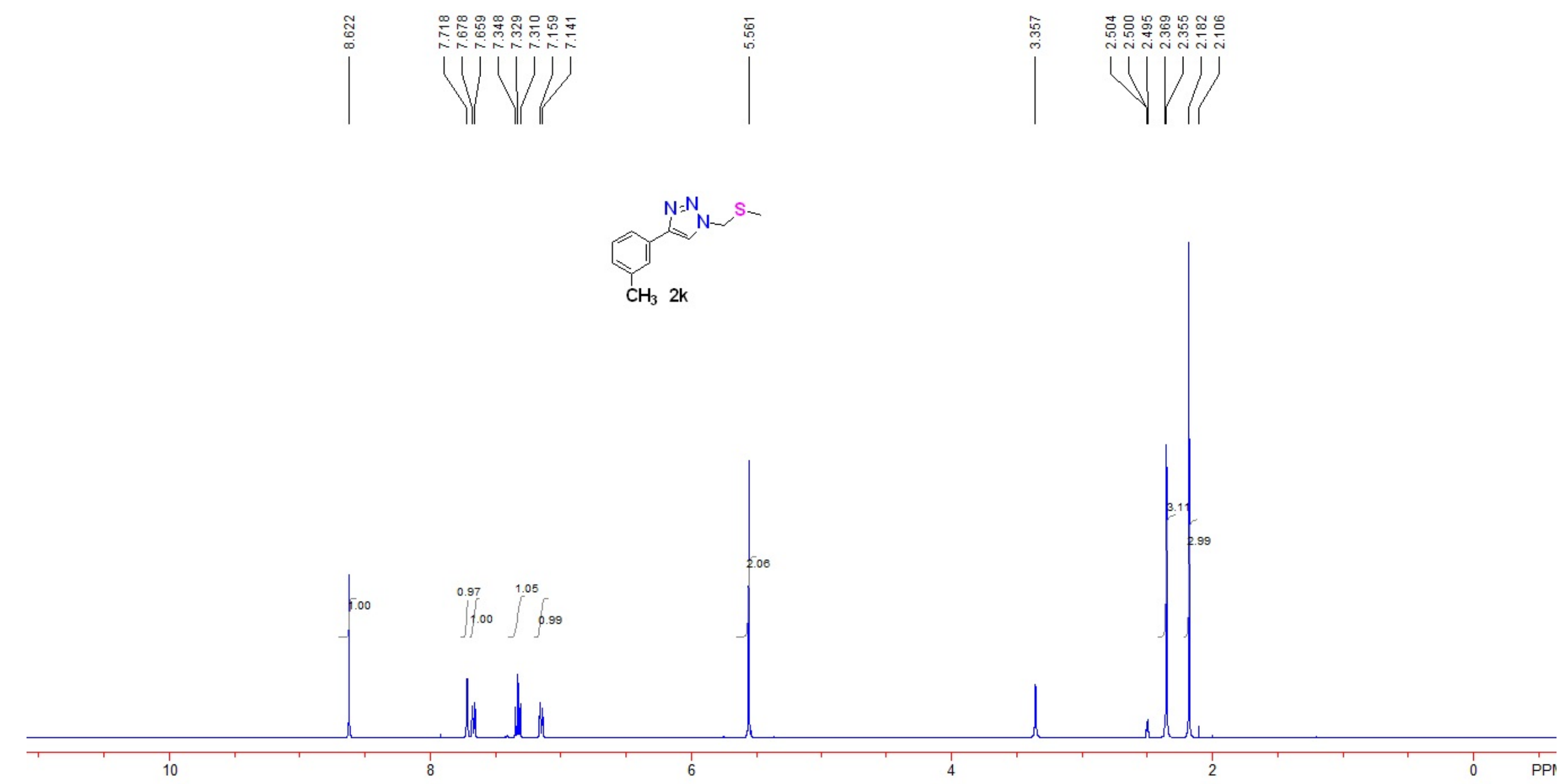



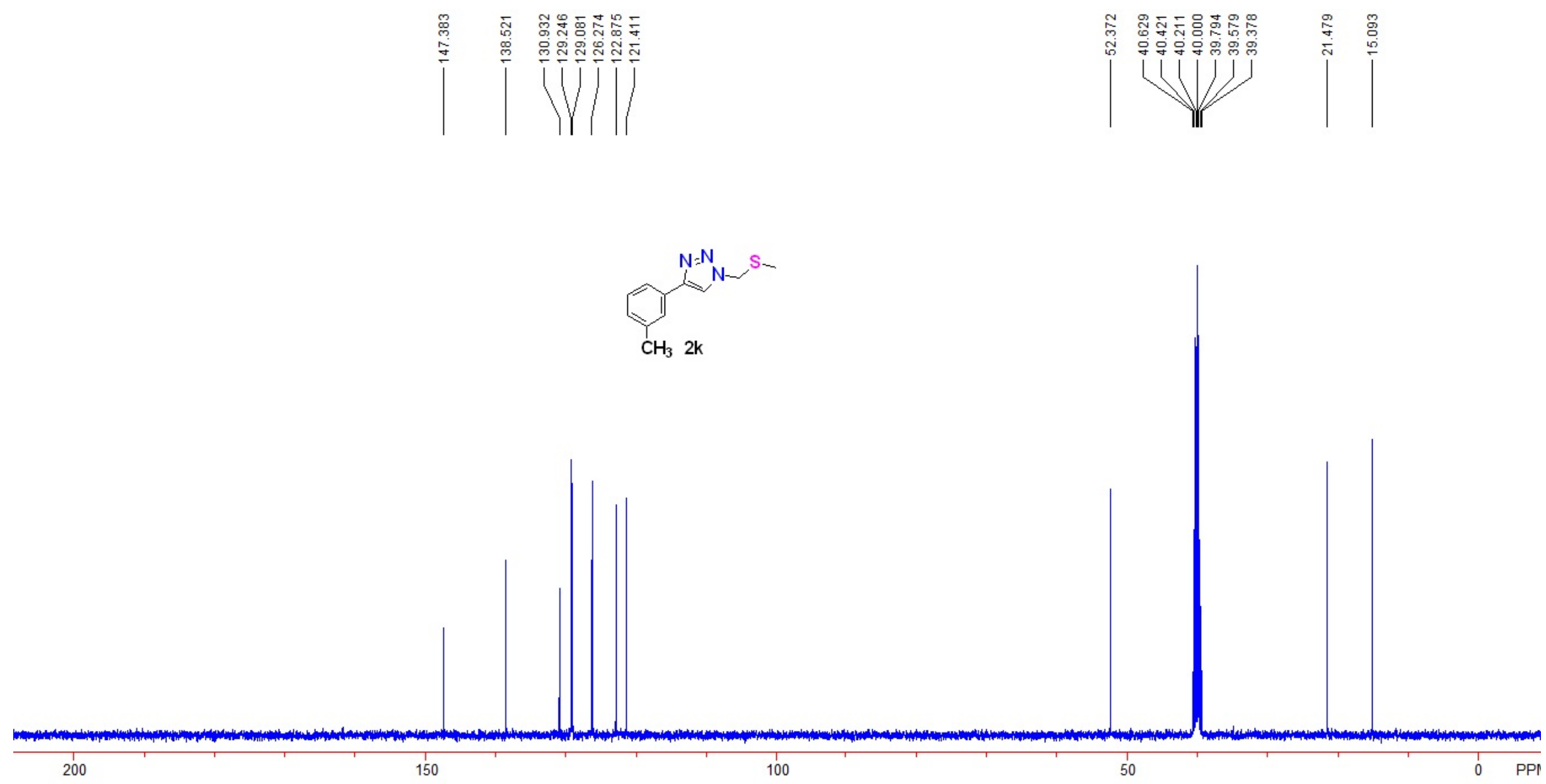


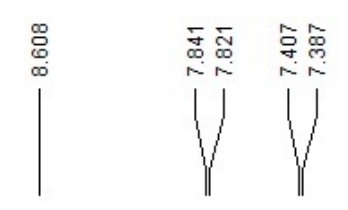

$\mid$
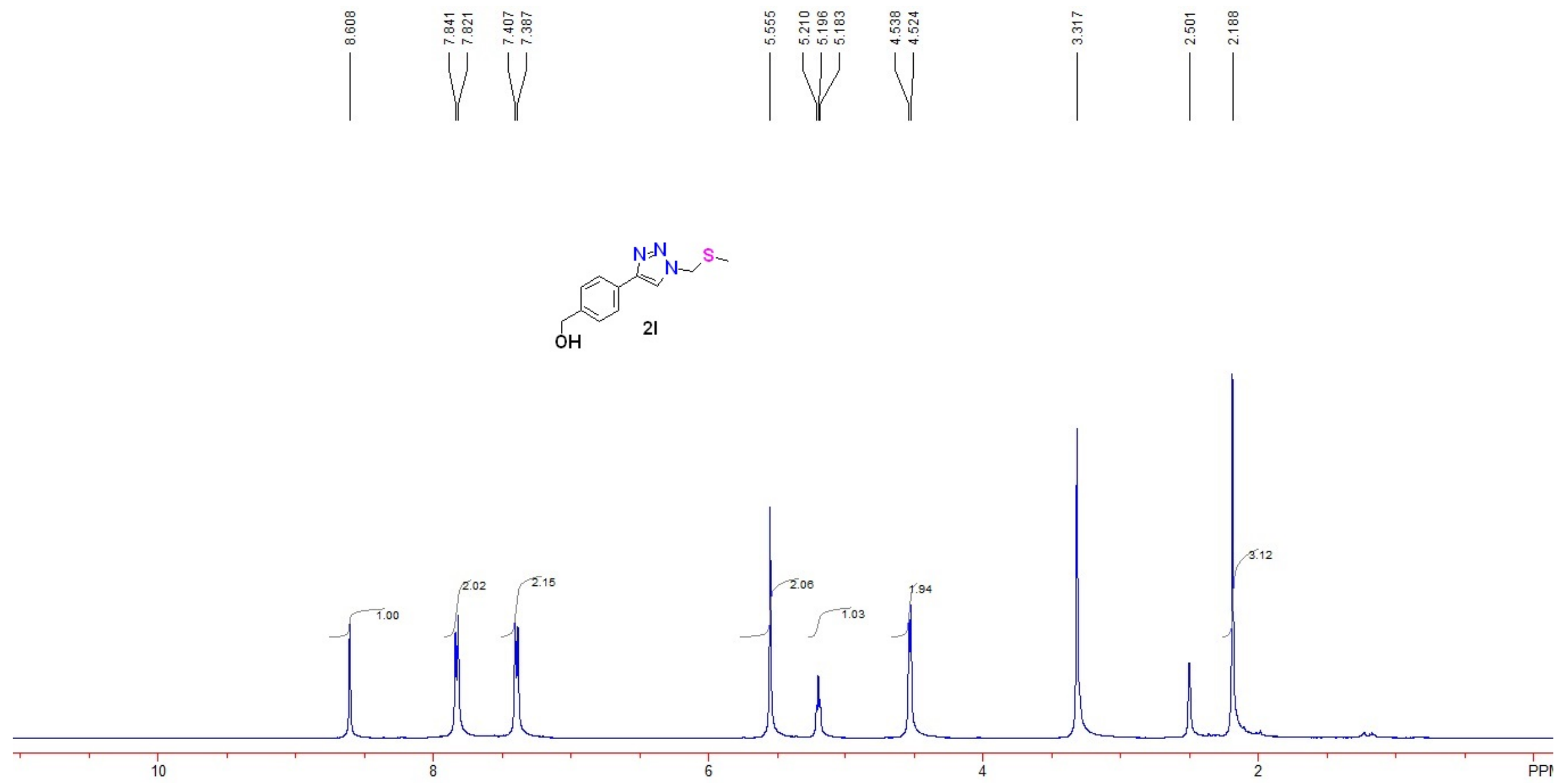

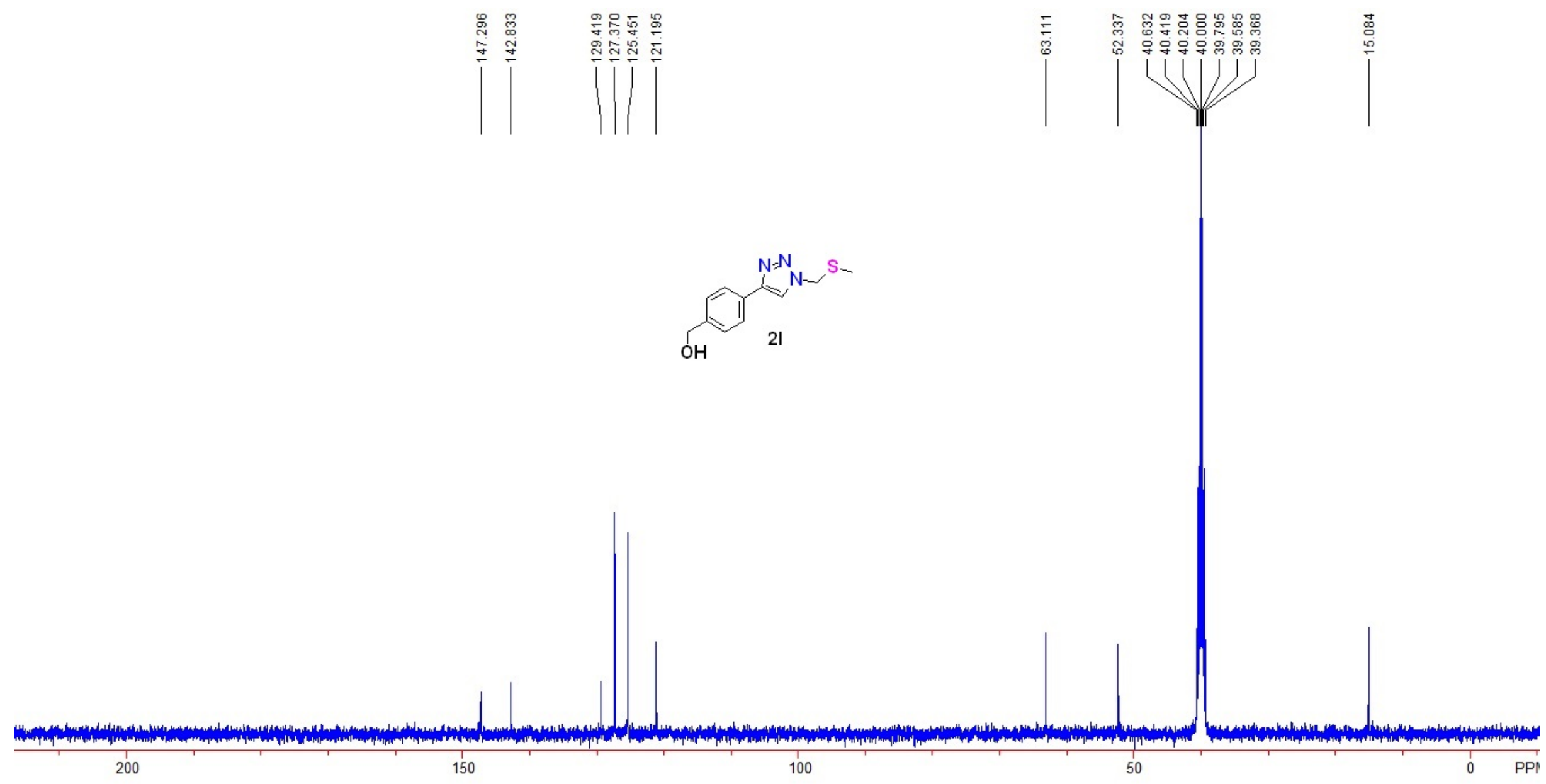

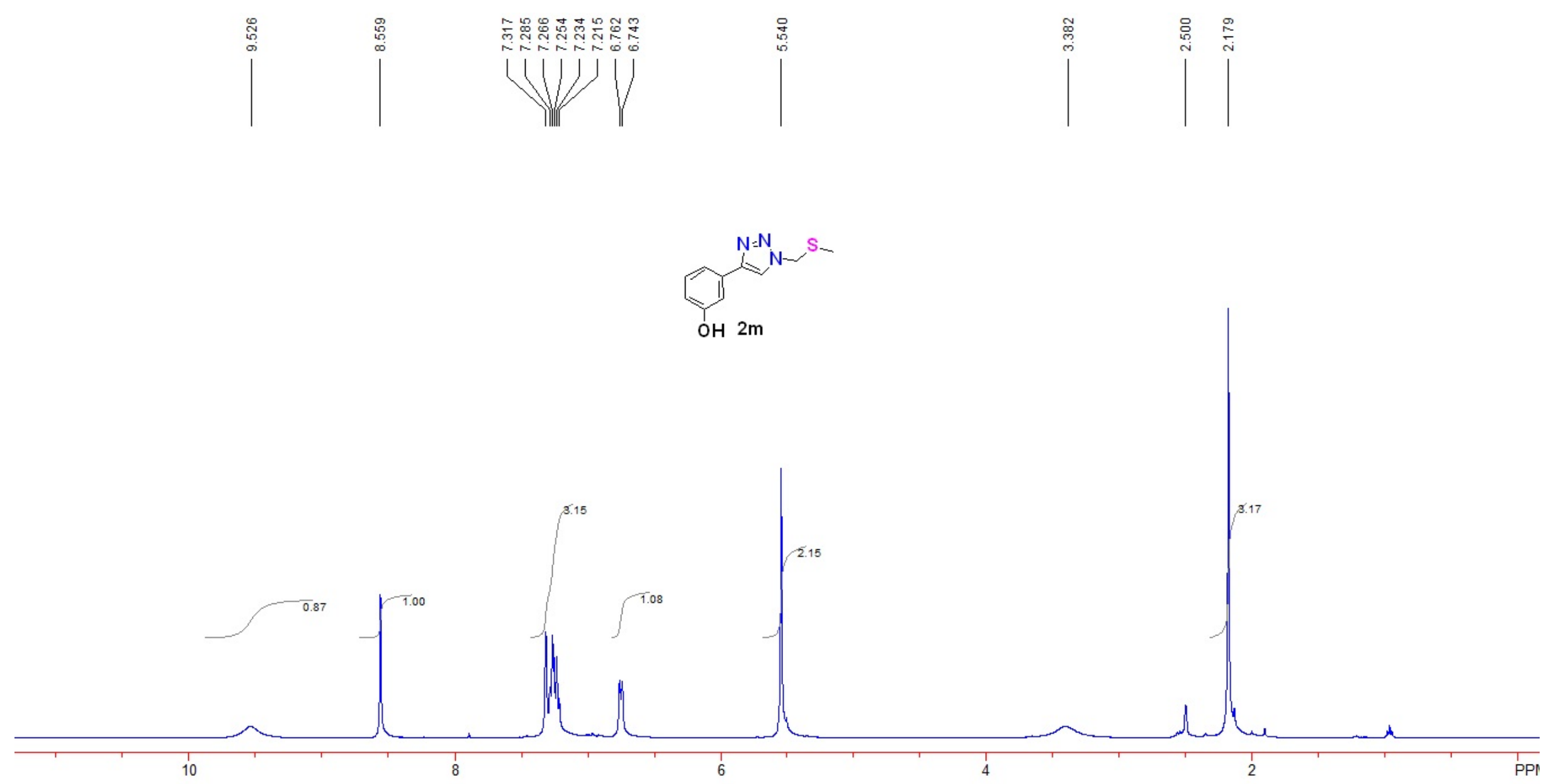


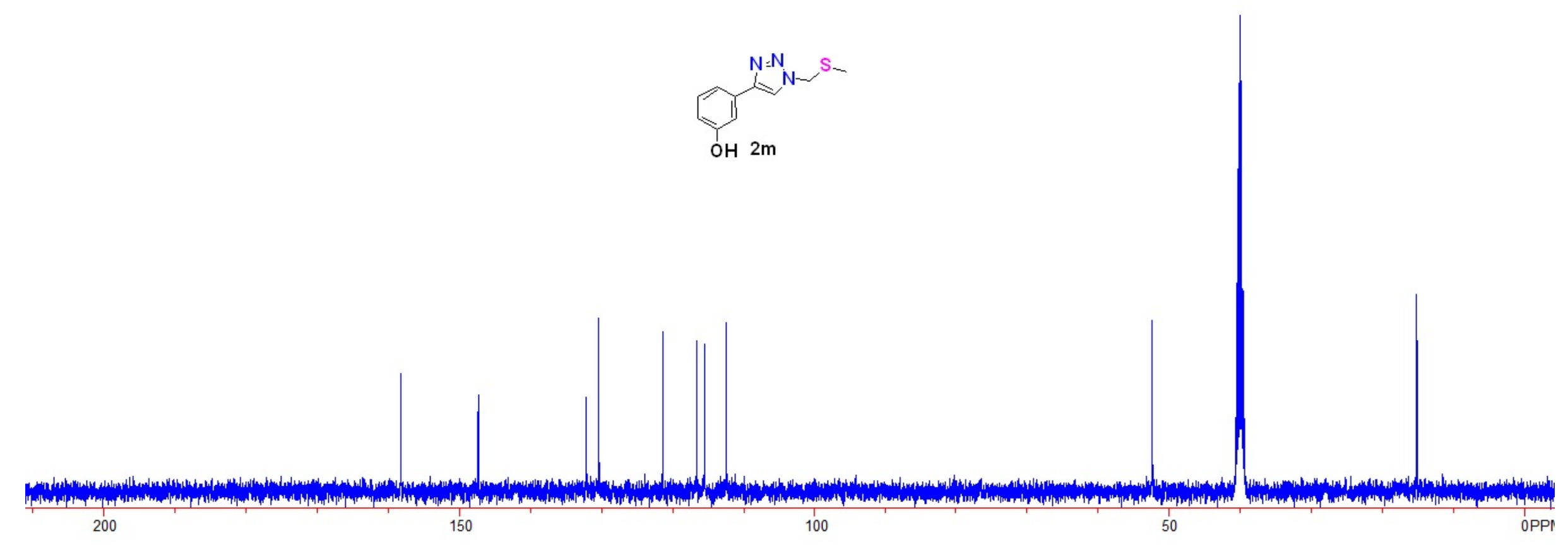




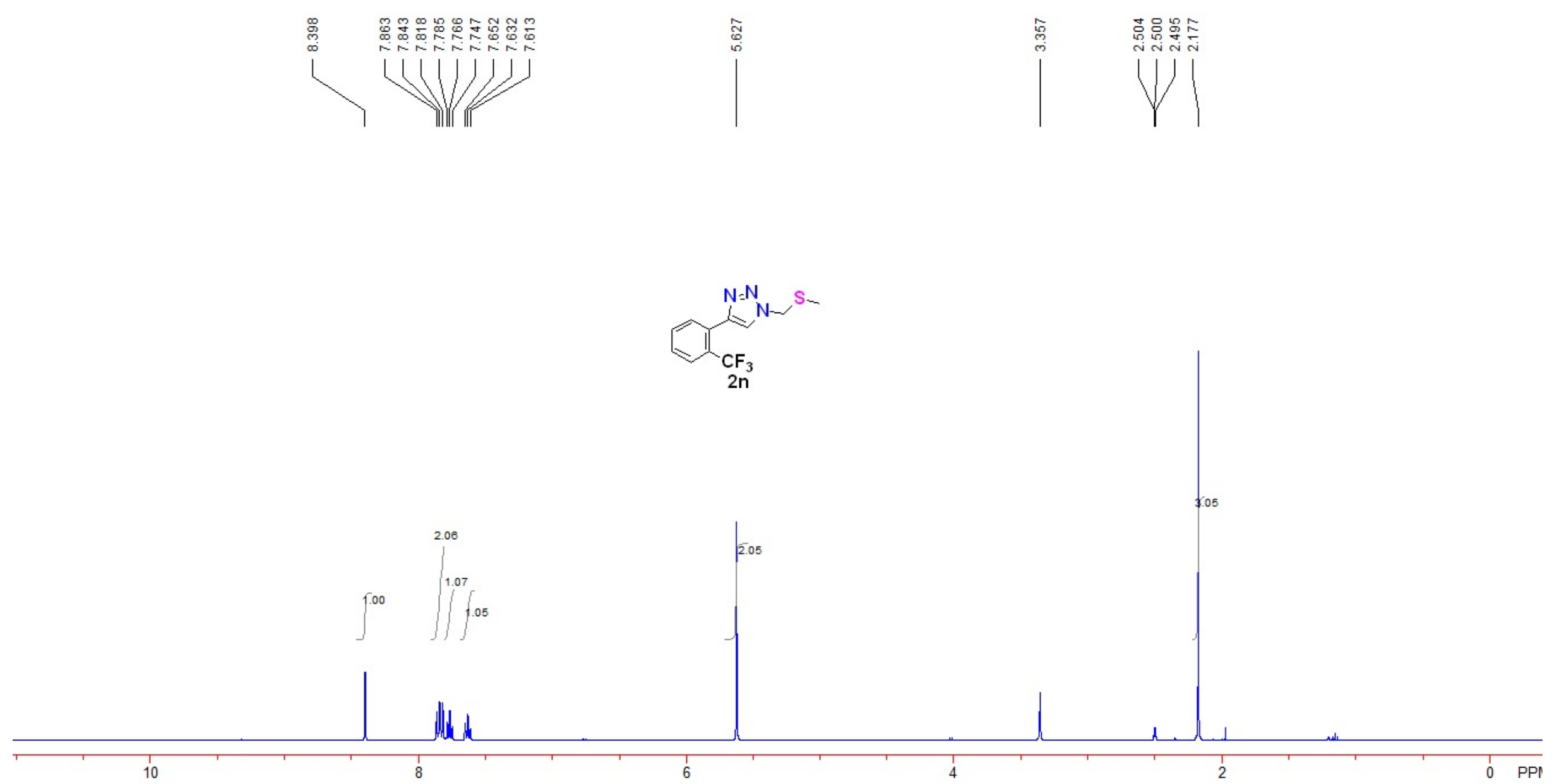




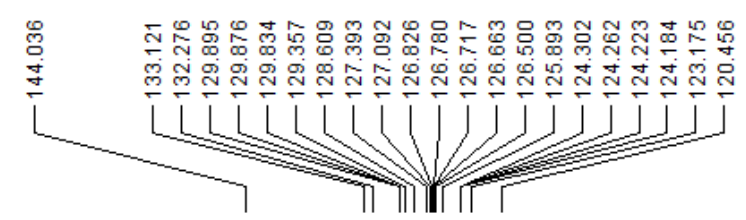

$\mathrm{N}=\mathrm{N}$

$\mathrm{CF}_{3}$

$2 n$

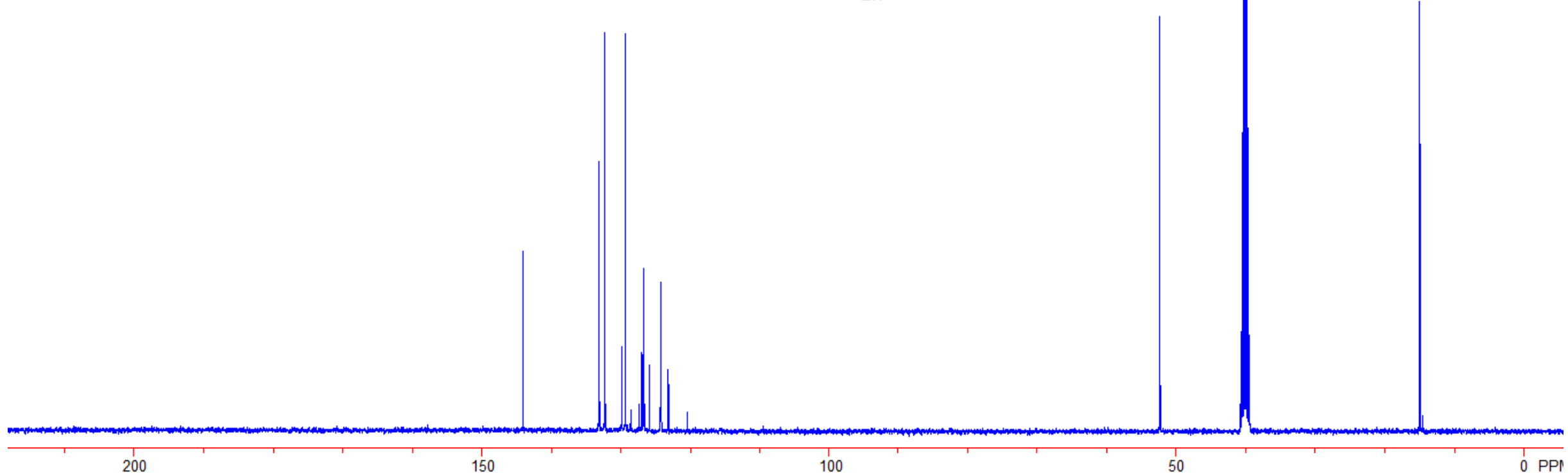



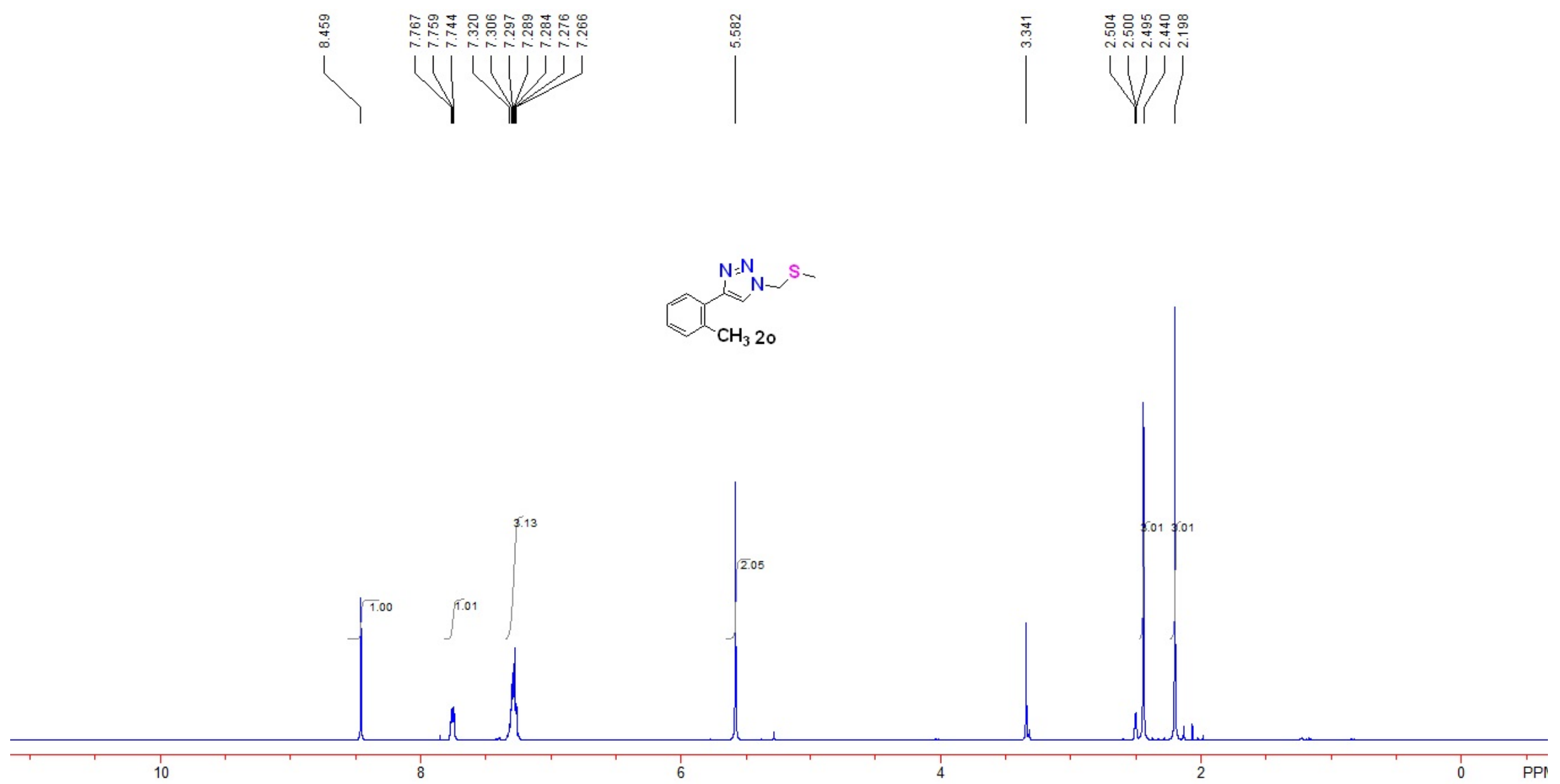

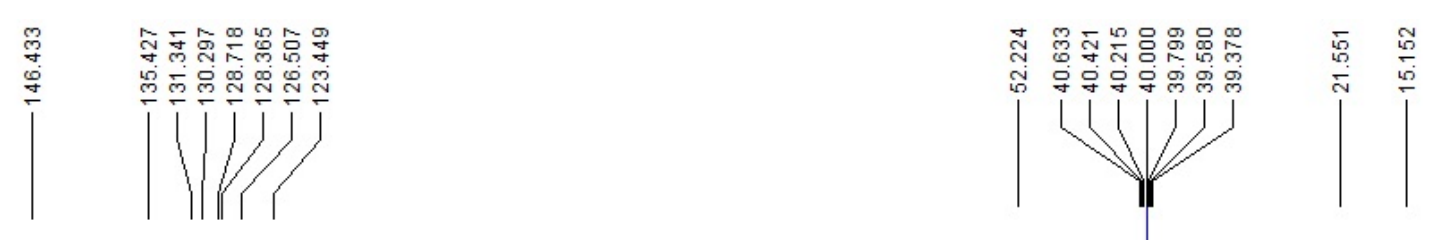

$$
\overbrace{\mathrm{CH}_{3} 2 \mathrm{O}}^{\mathrm{N}=\mathrm{N}}
$$

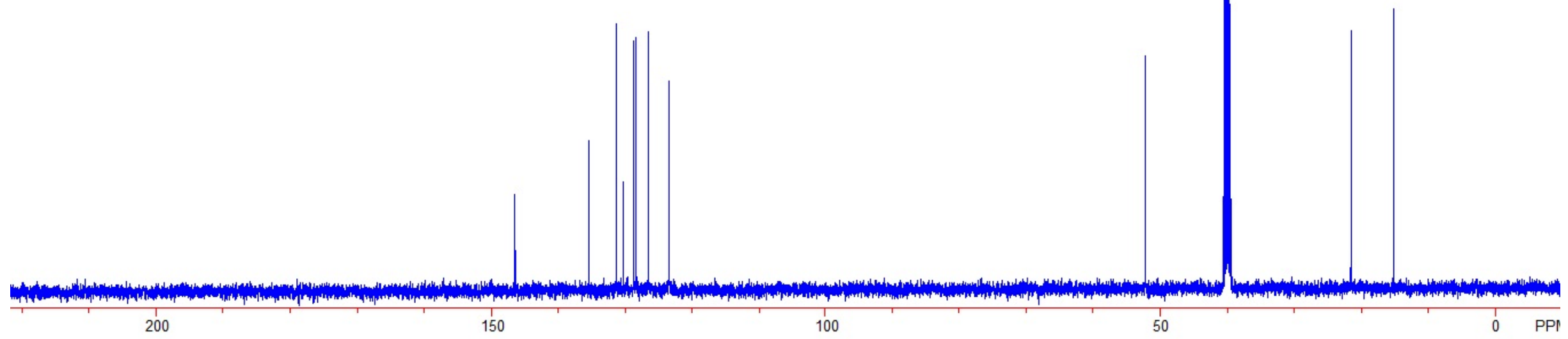



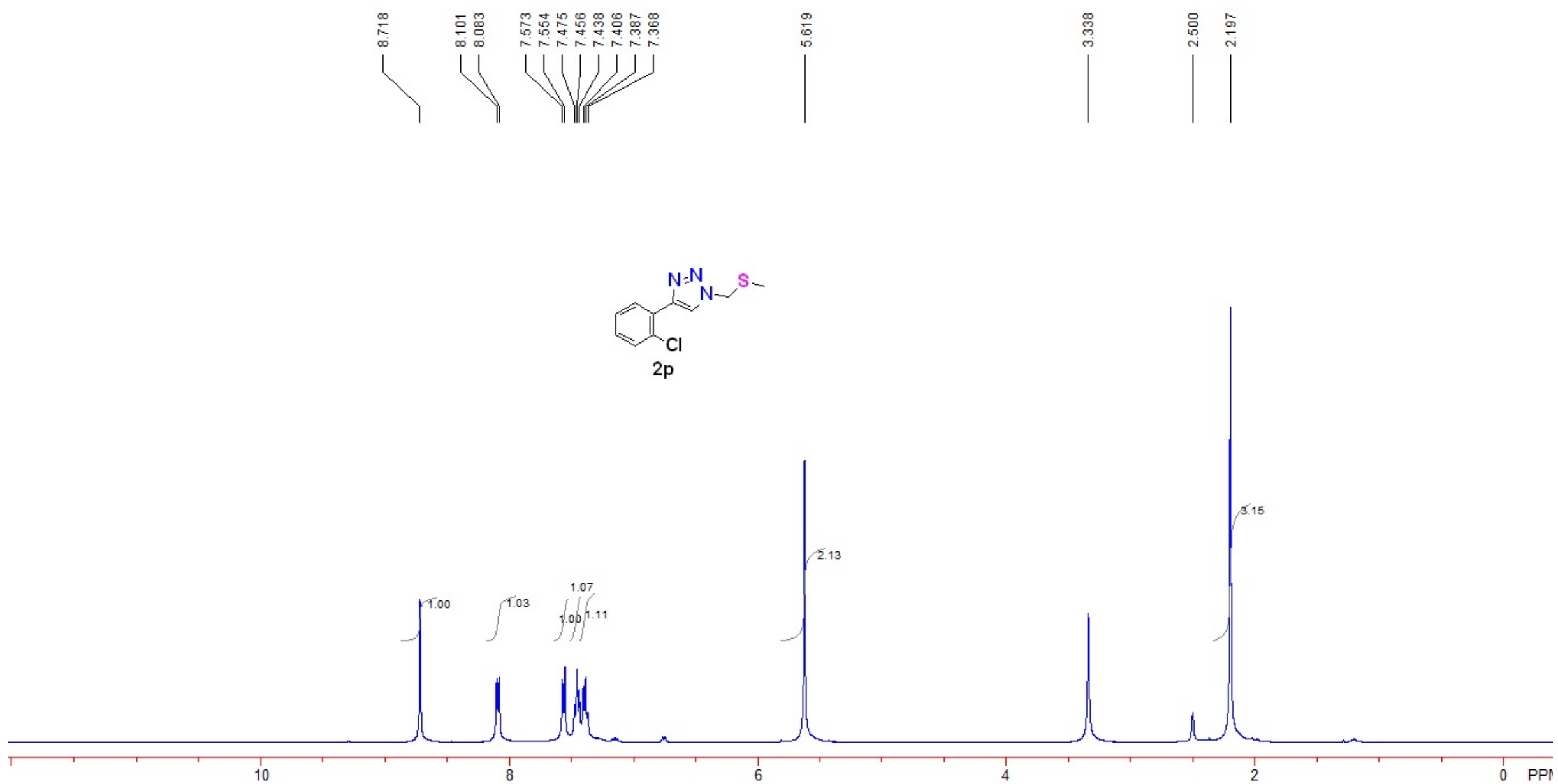

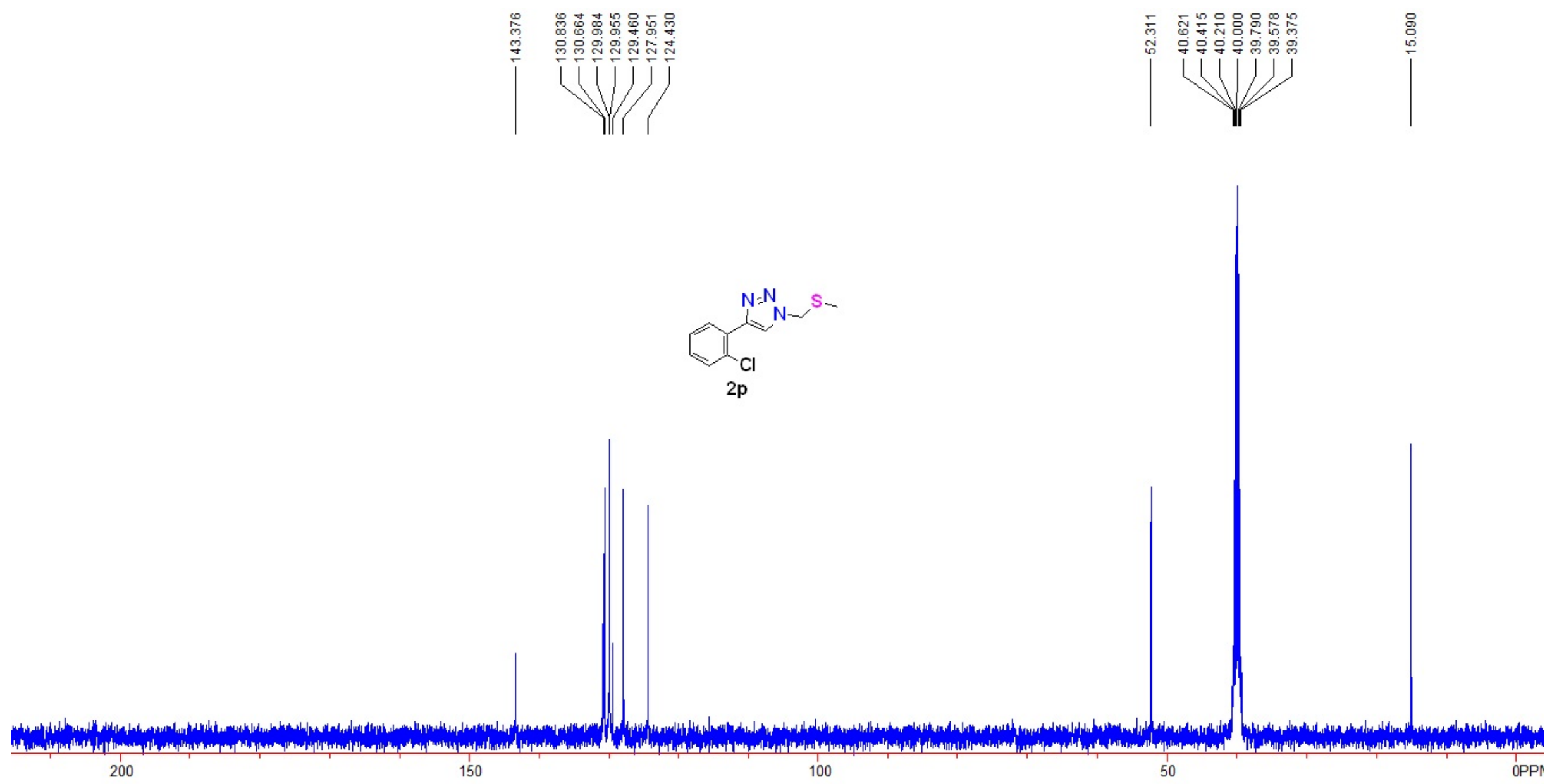

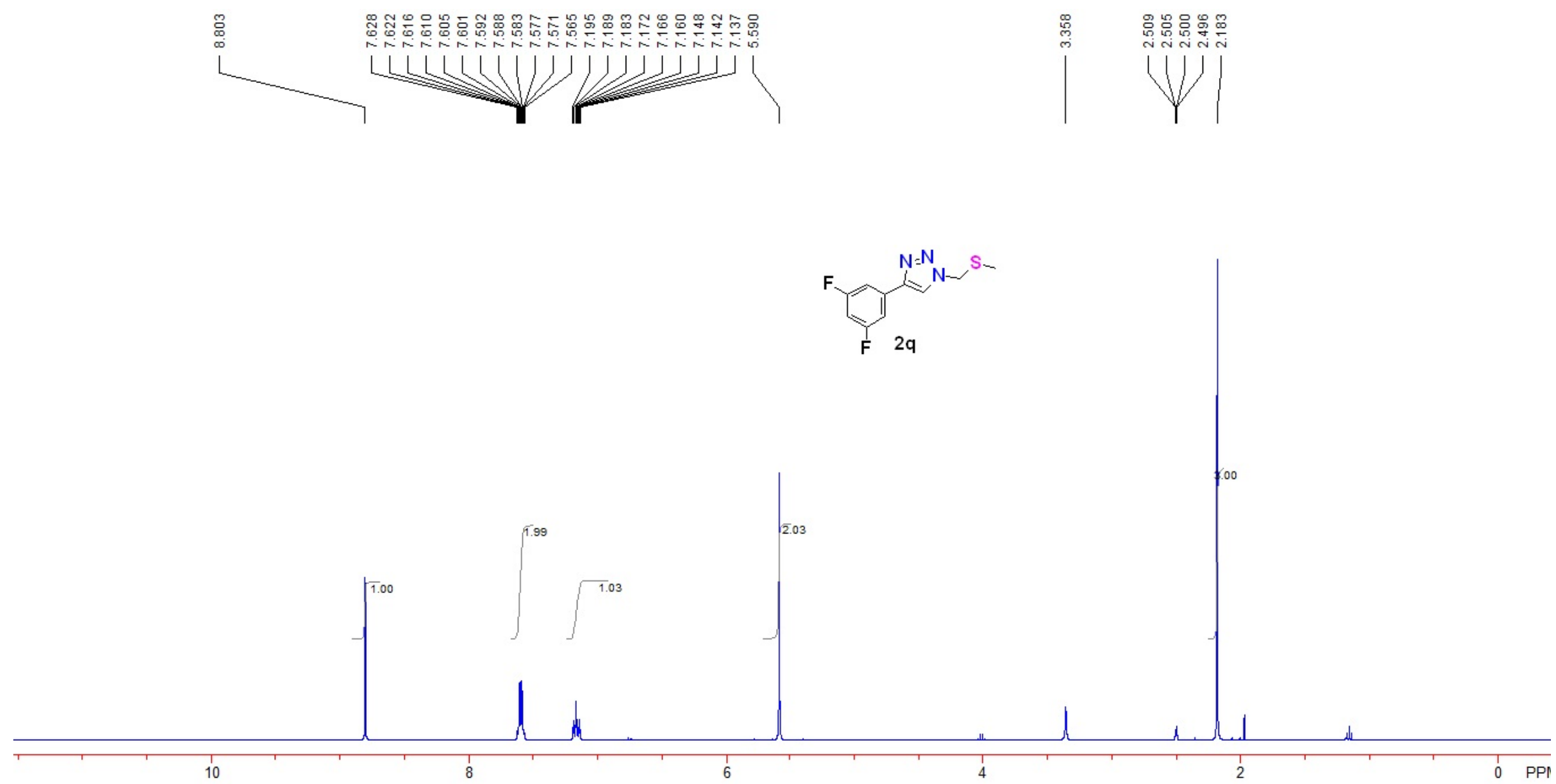

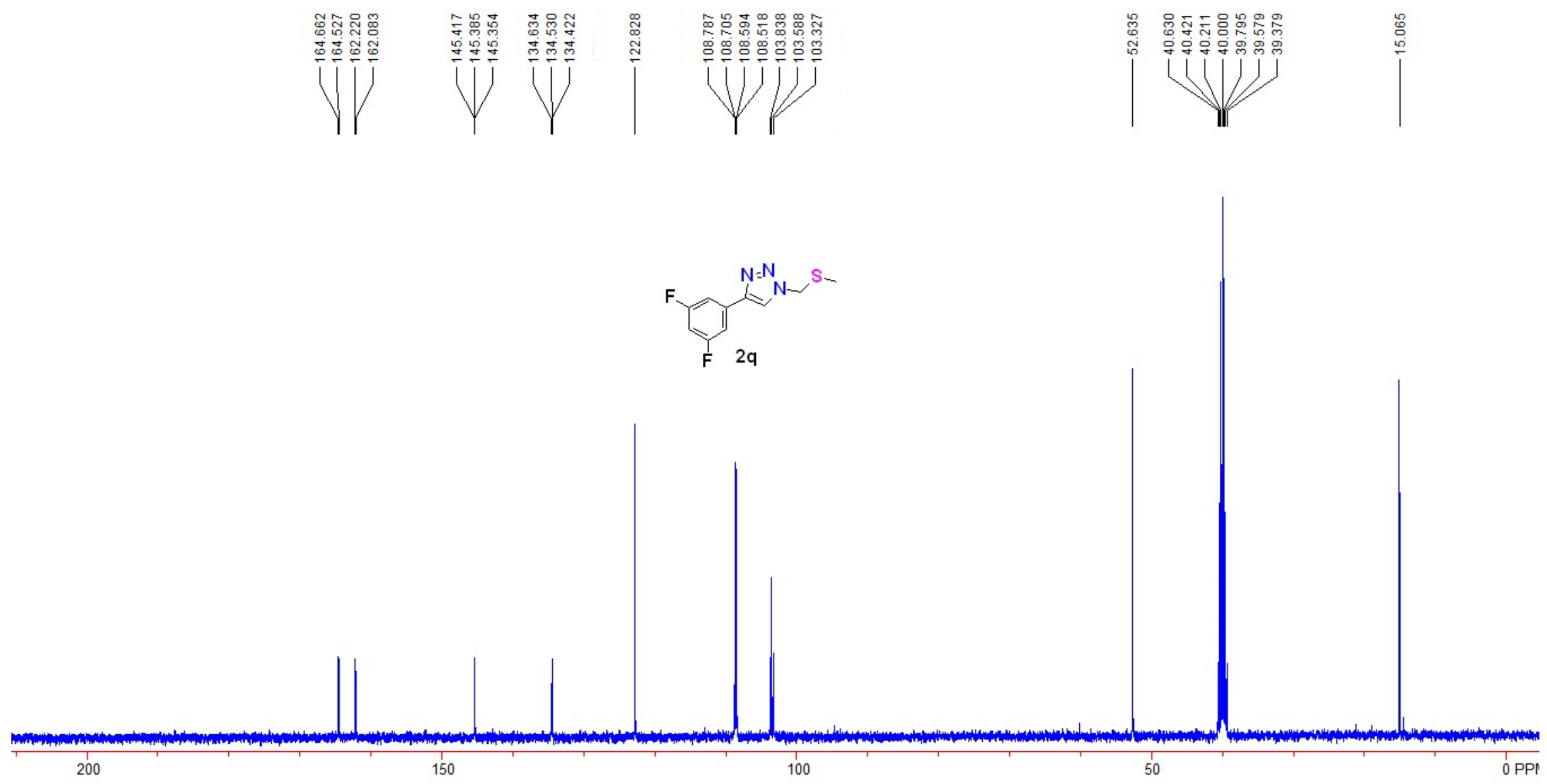


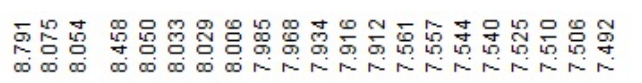

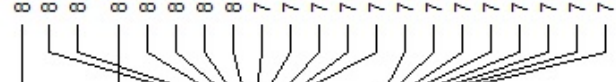

$\underset{\substack{0 \\ 0}}{\infty}$

anlif if

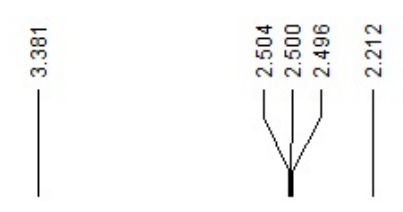

2

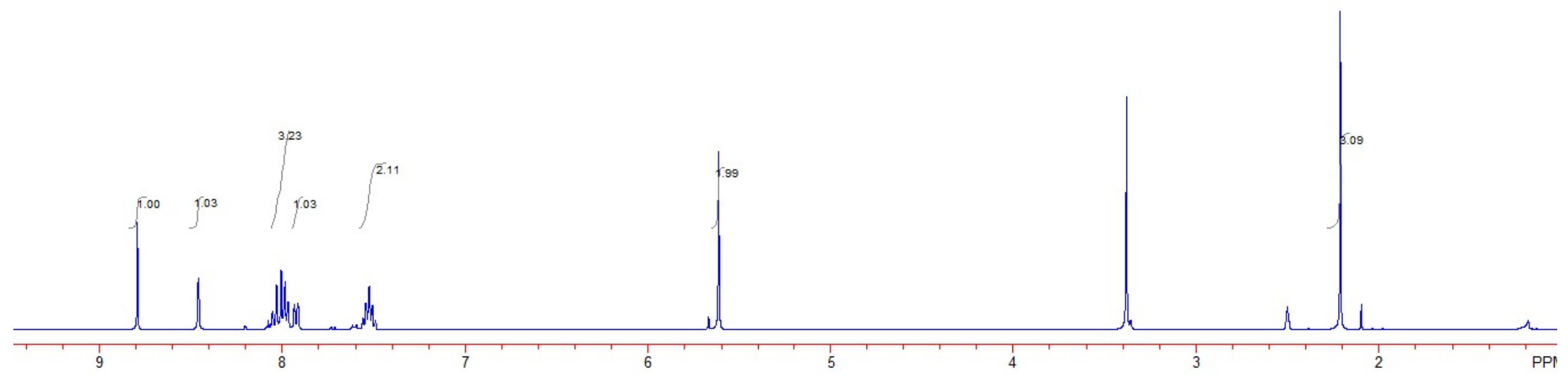




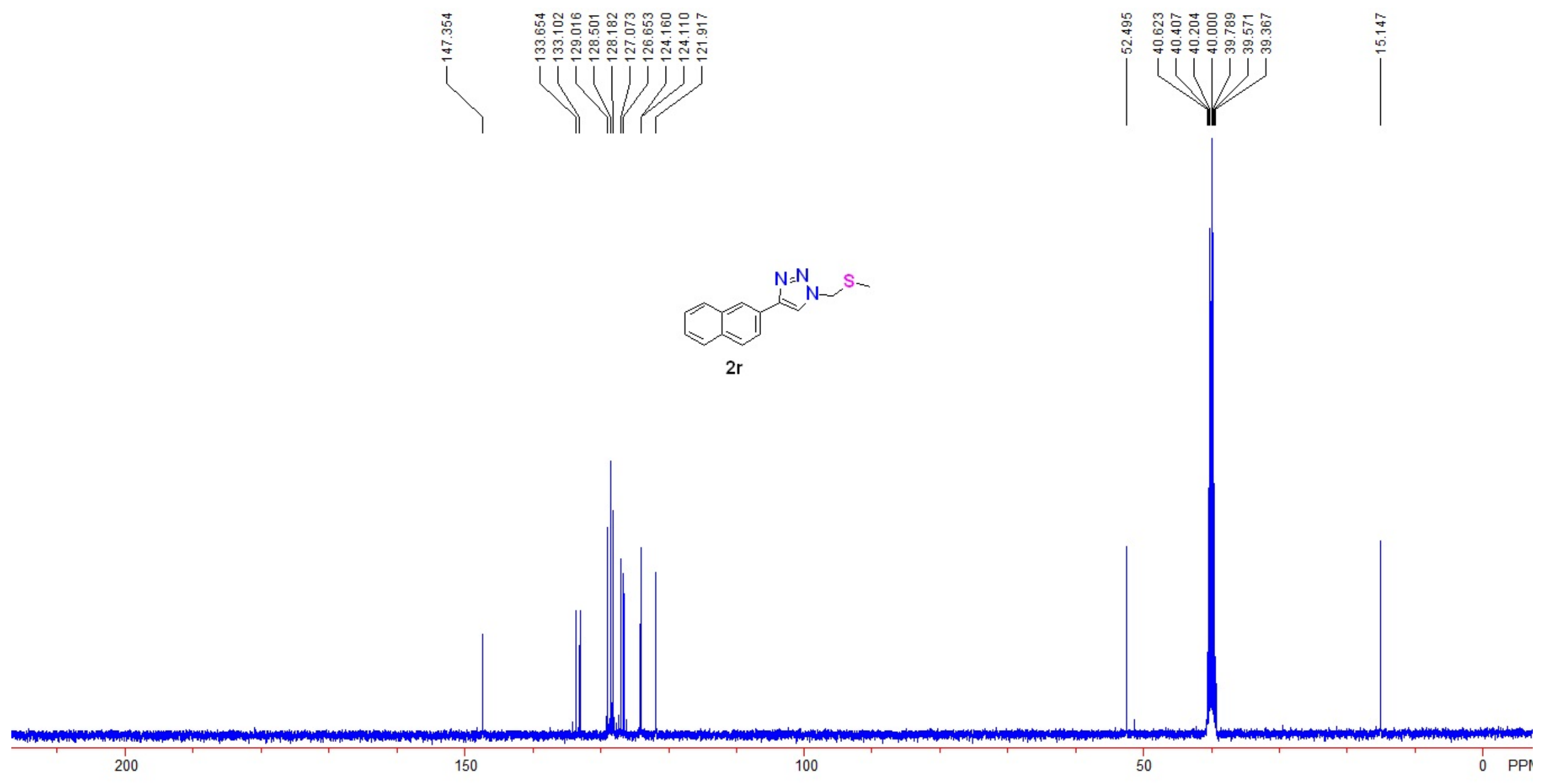



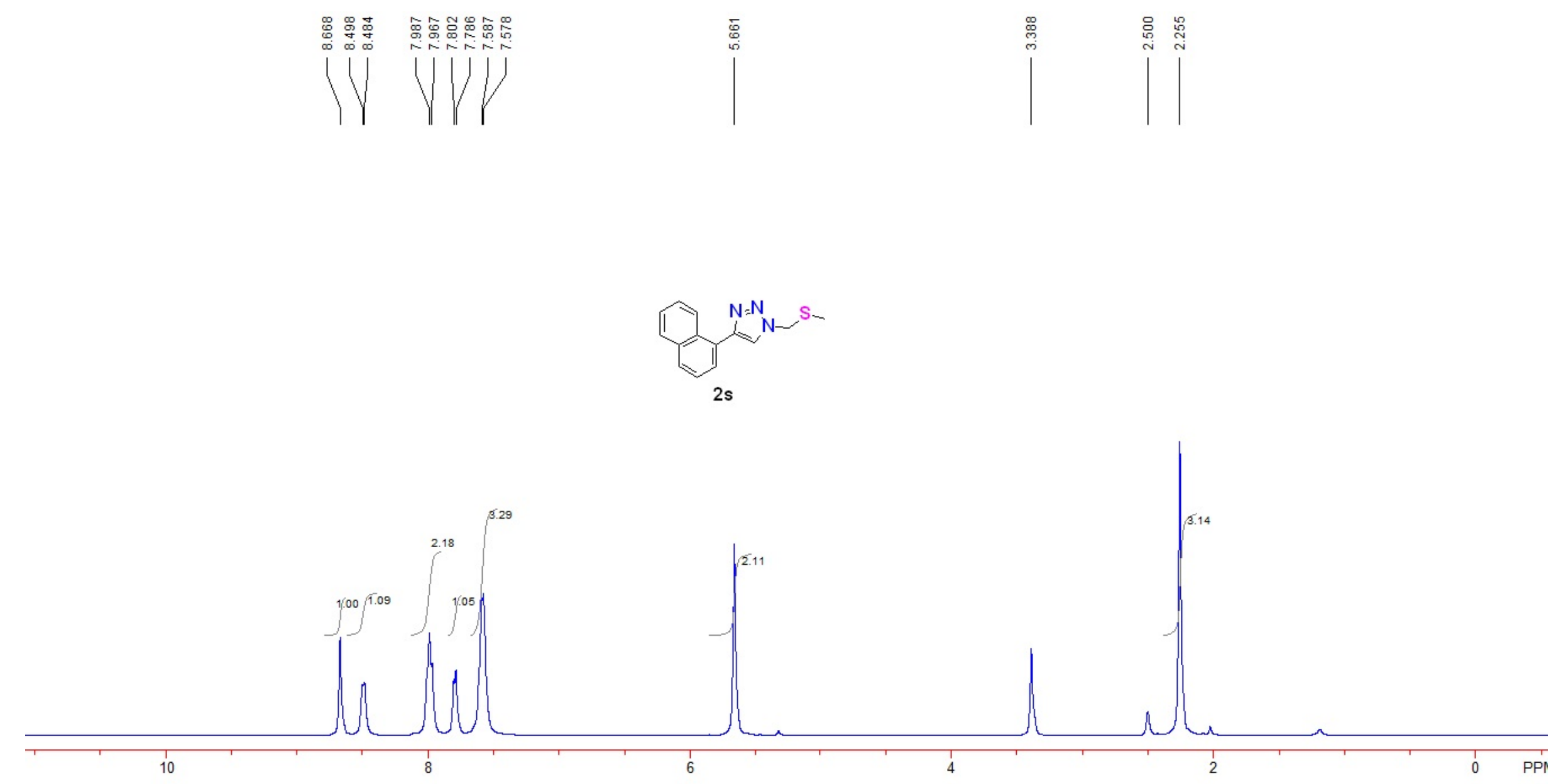

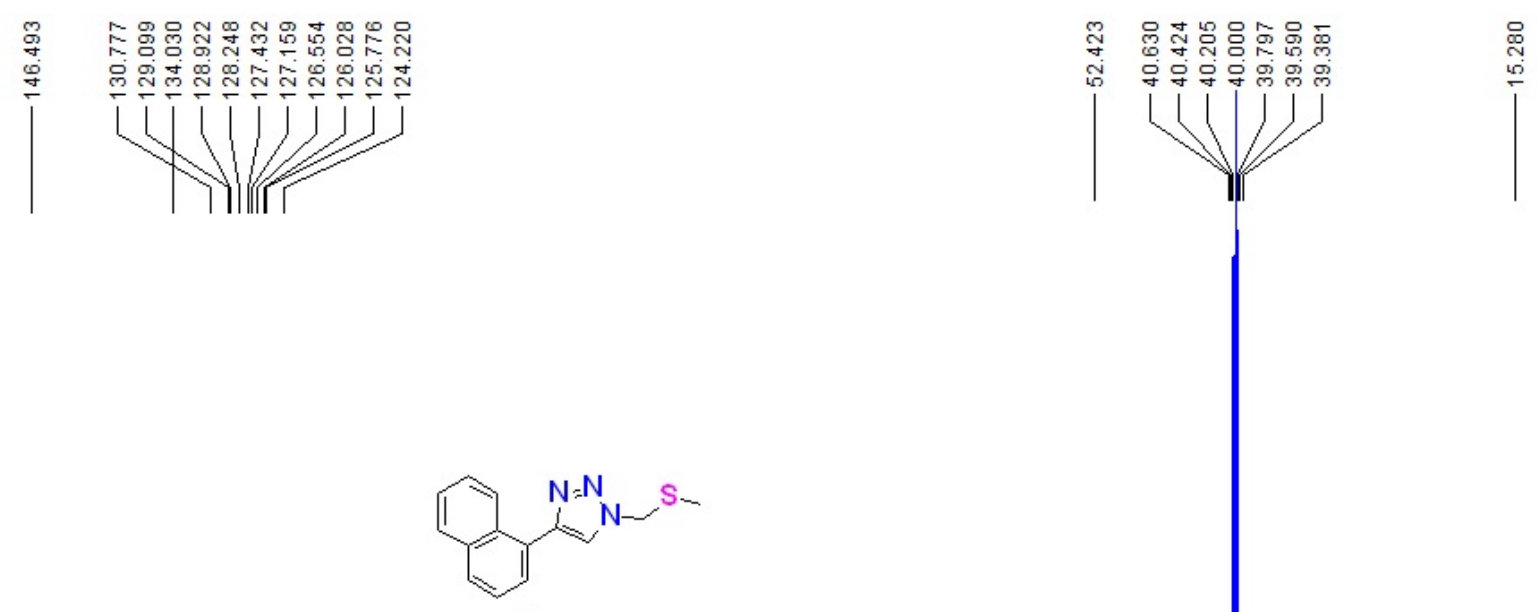

$2 s$

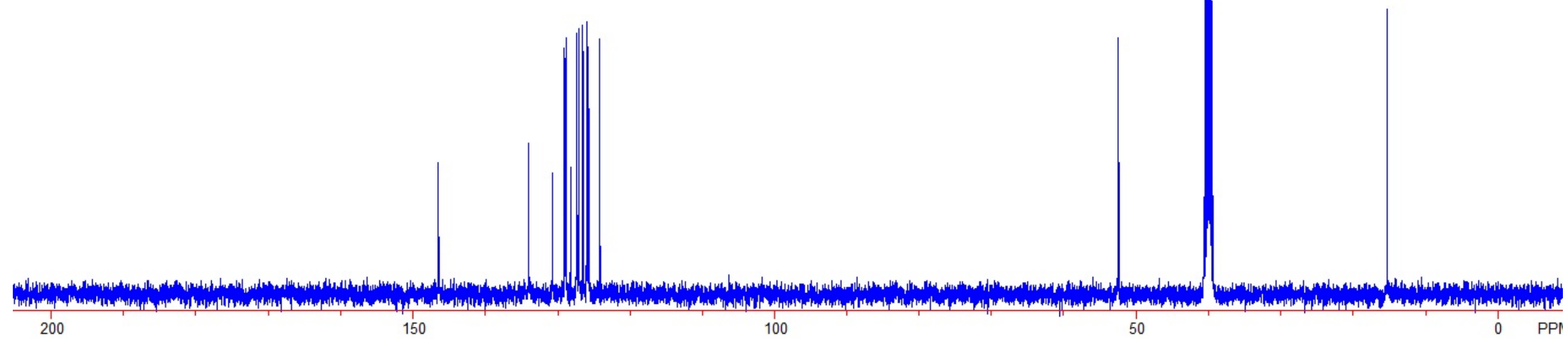




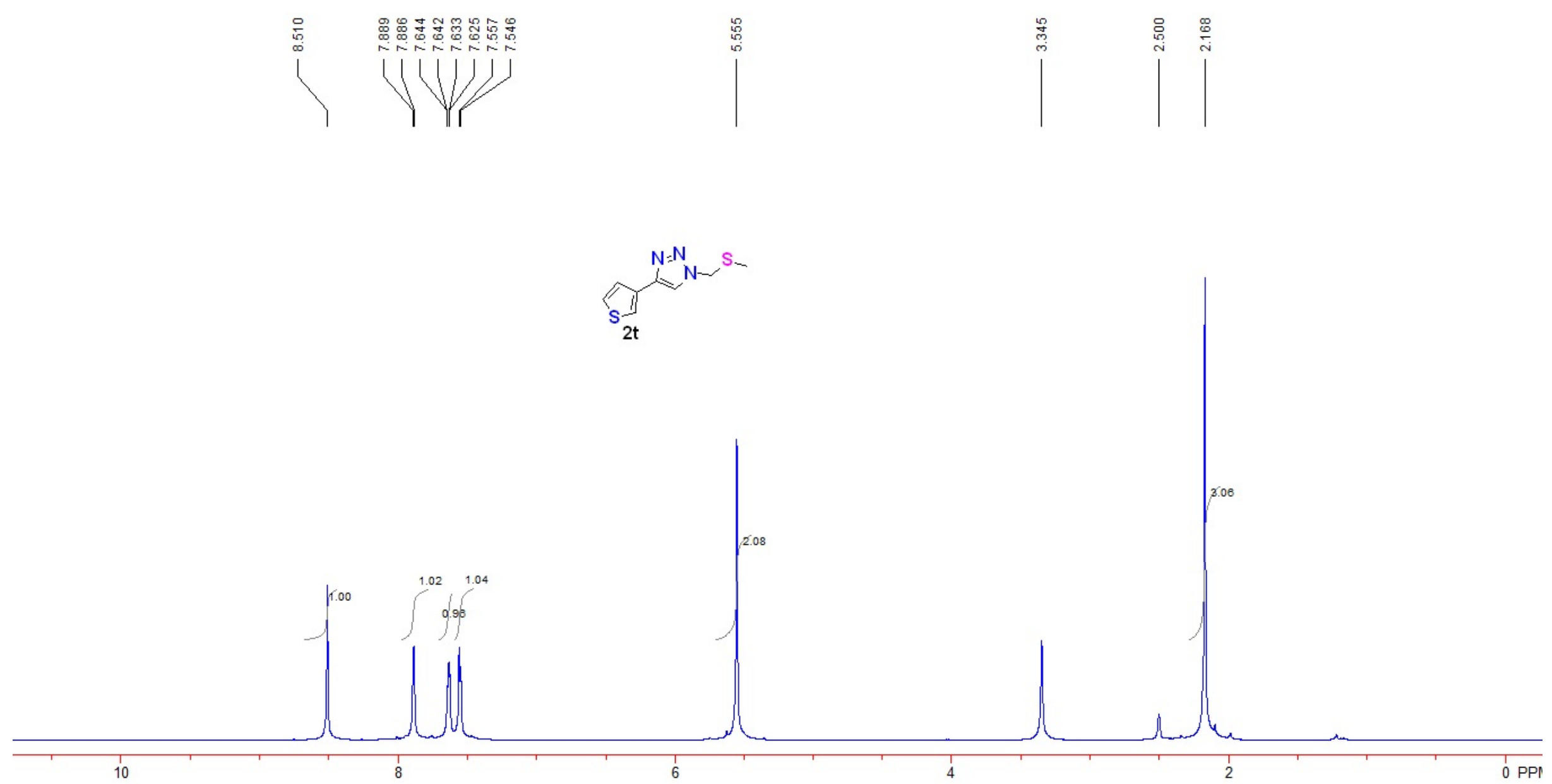



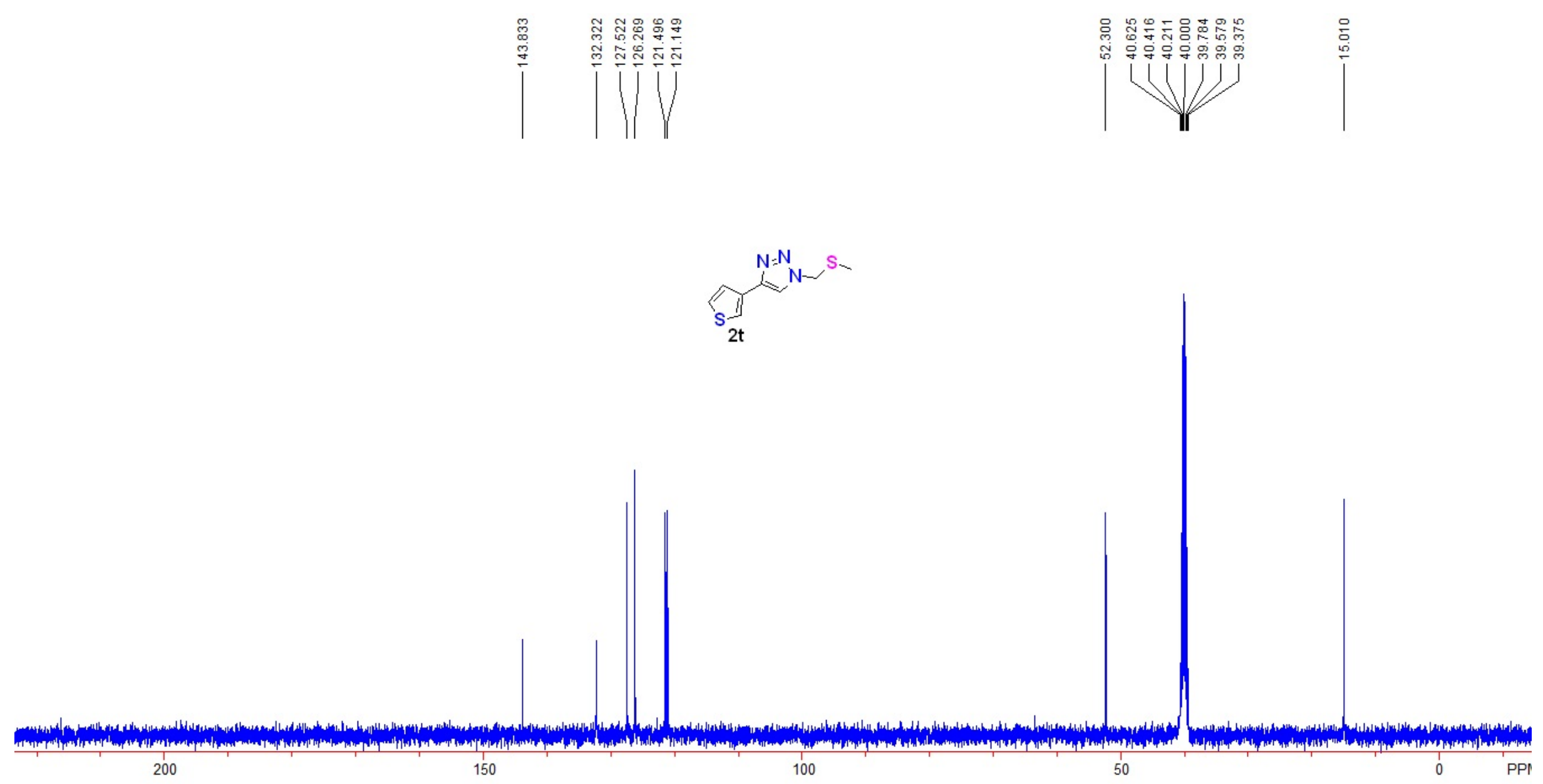

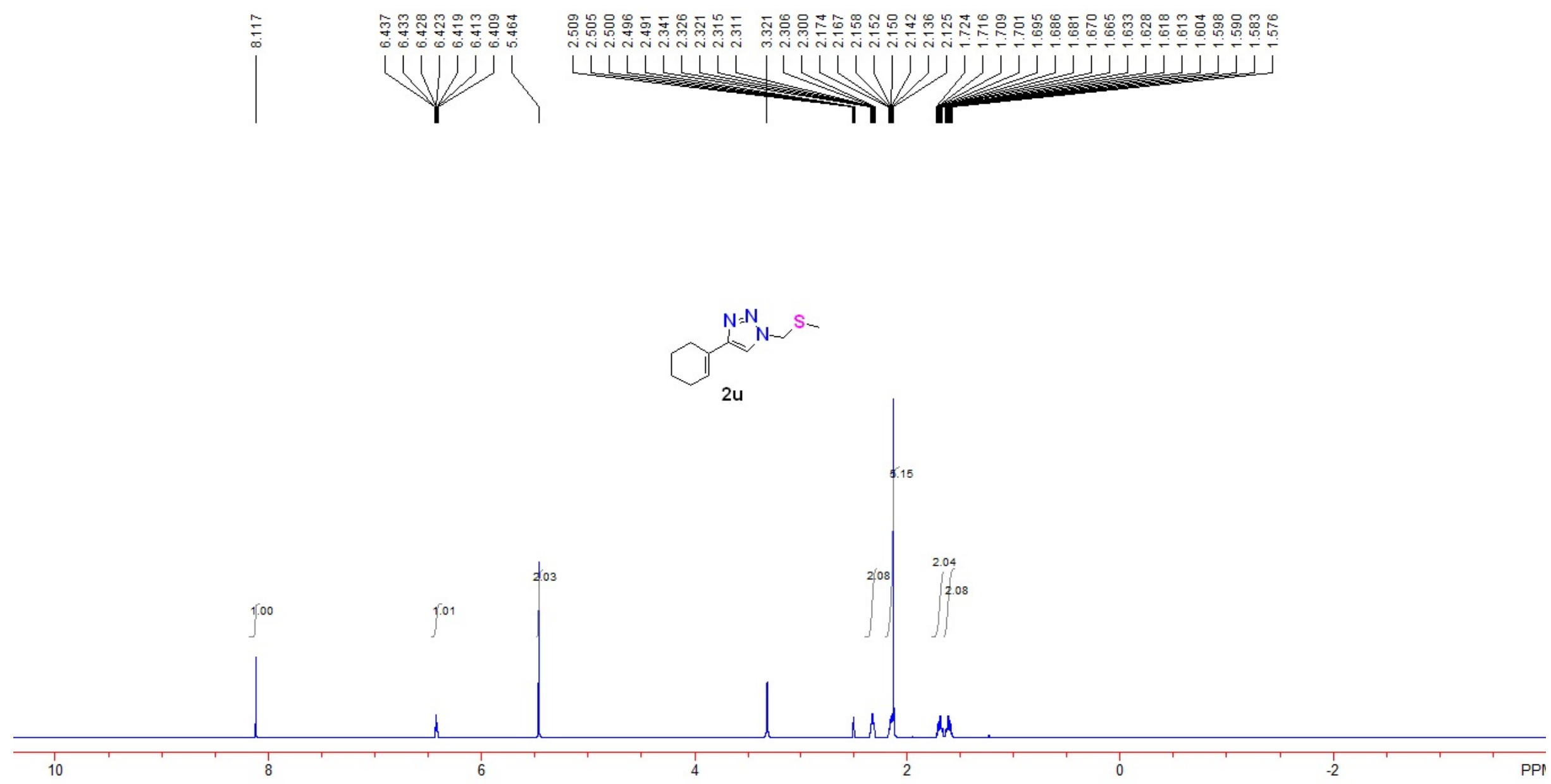


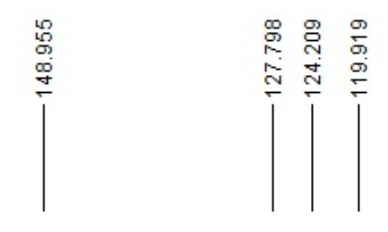

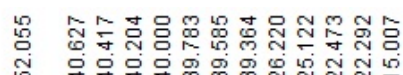

L

$\mathrm{N}_{\mathrm{N}}^{\mathrm{N}} \mathrm{S}$

$2 \mathrm{u}$

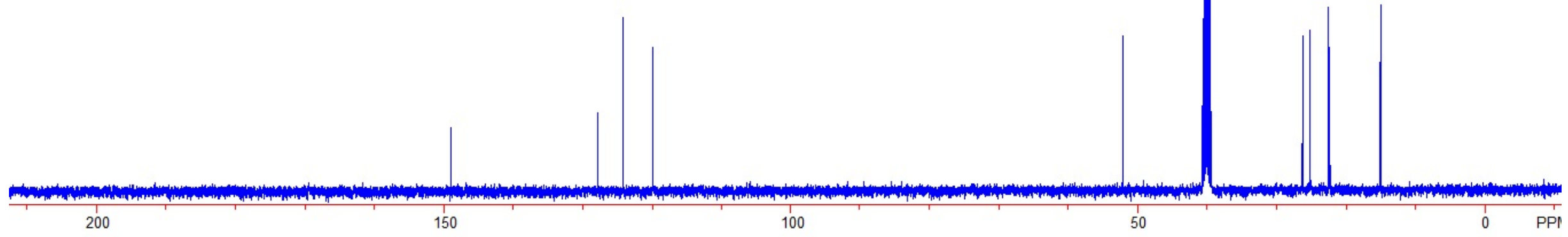



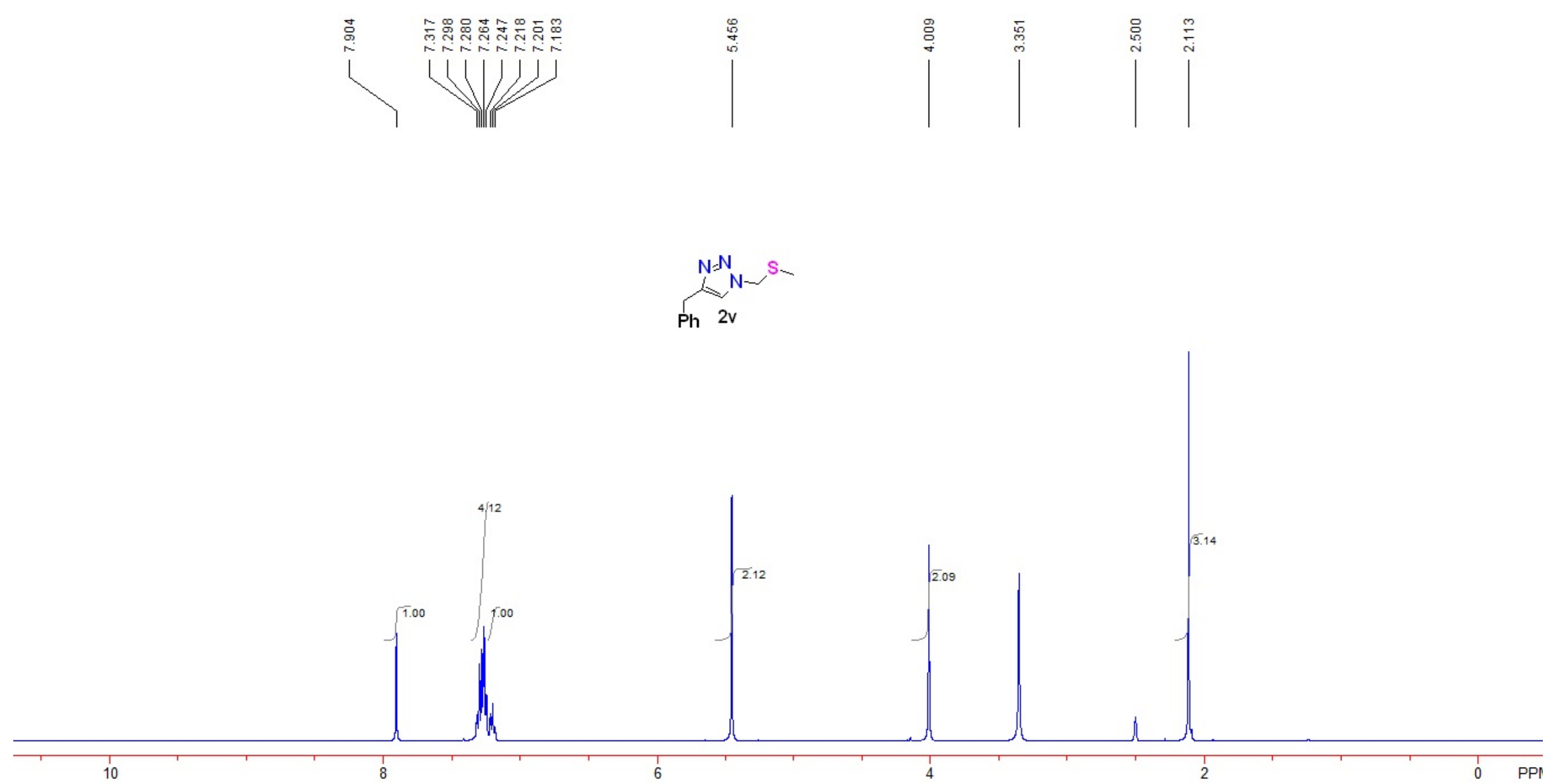

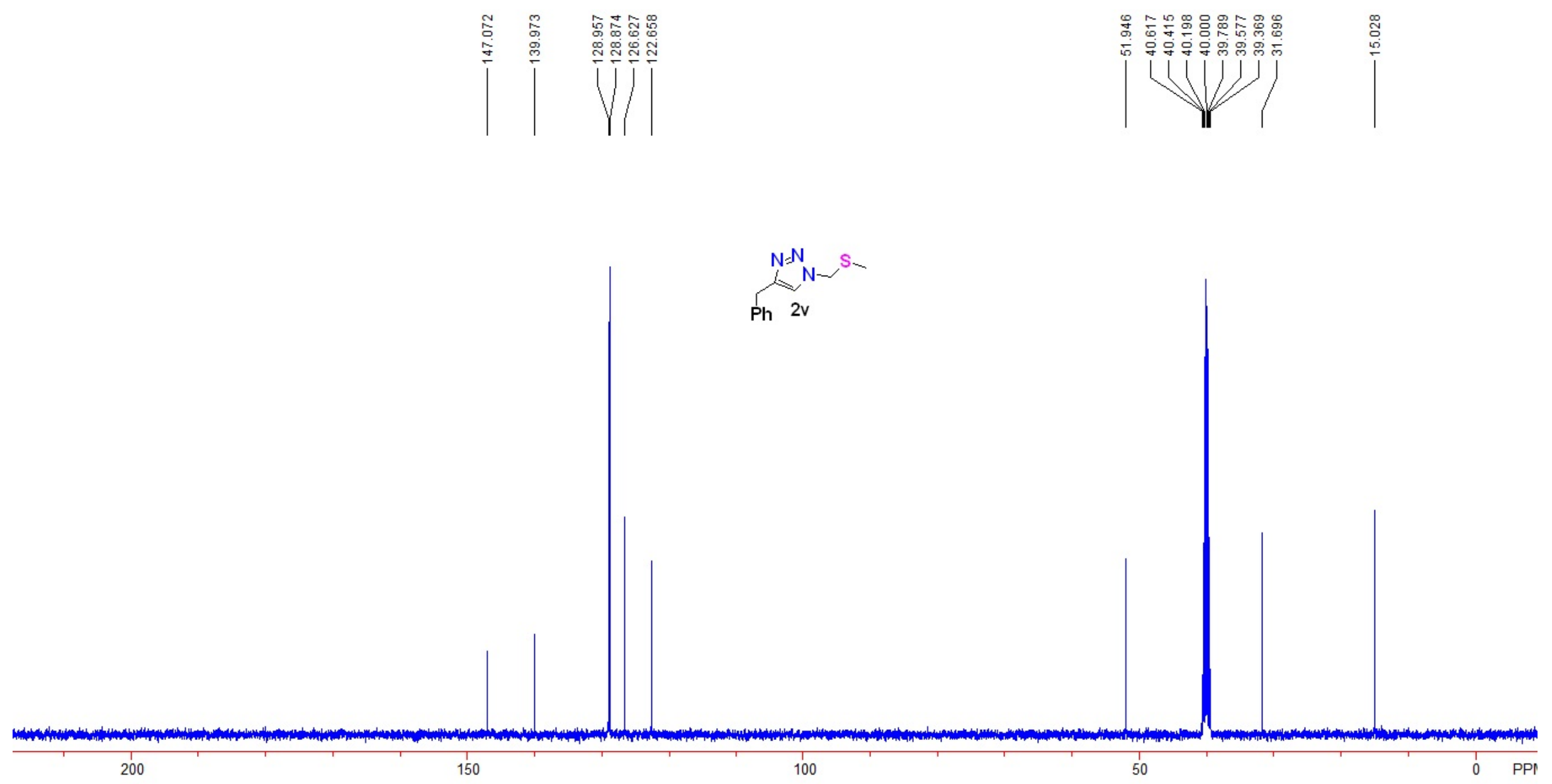


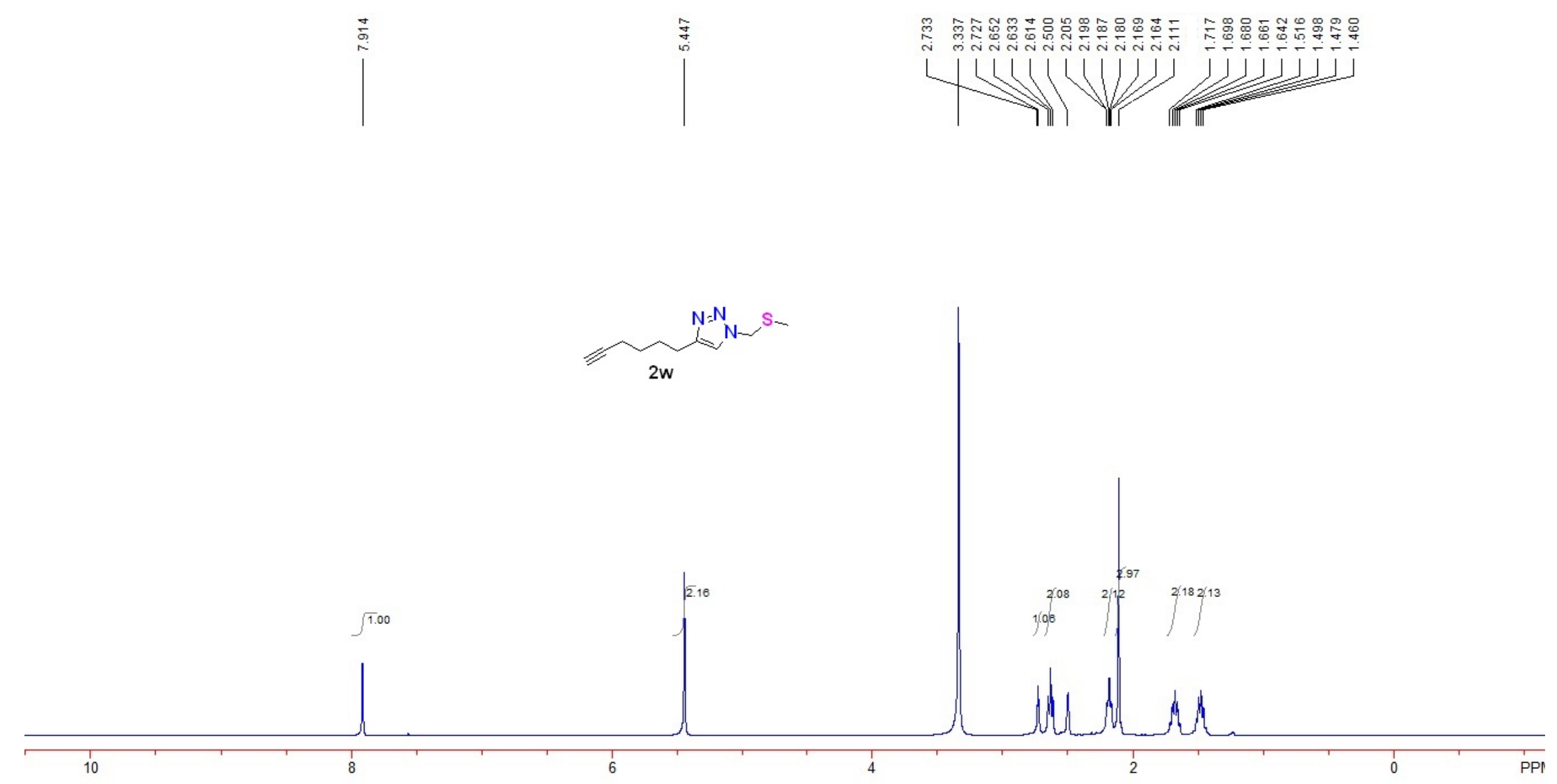




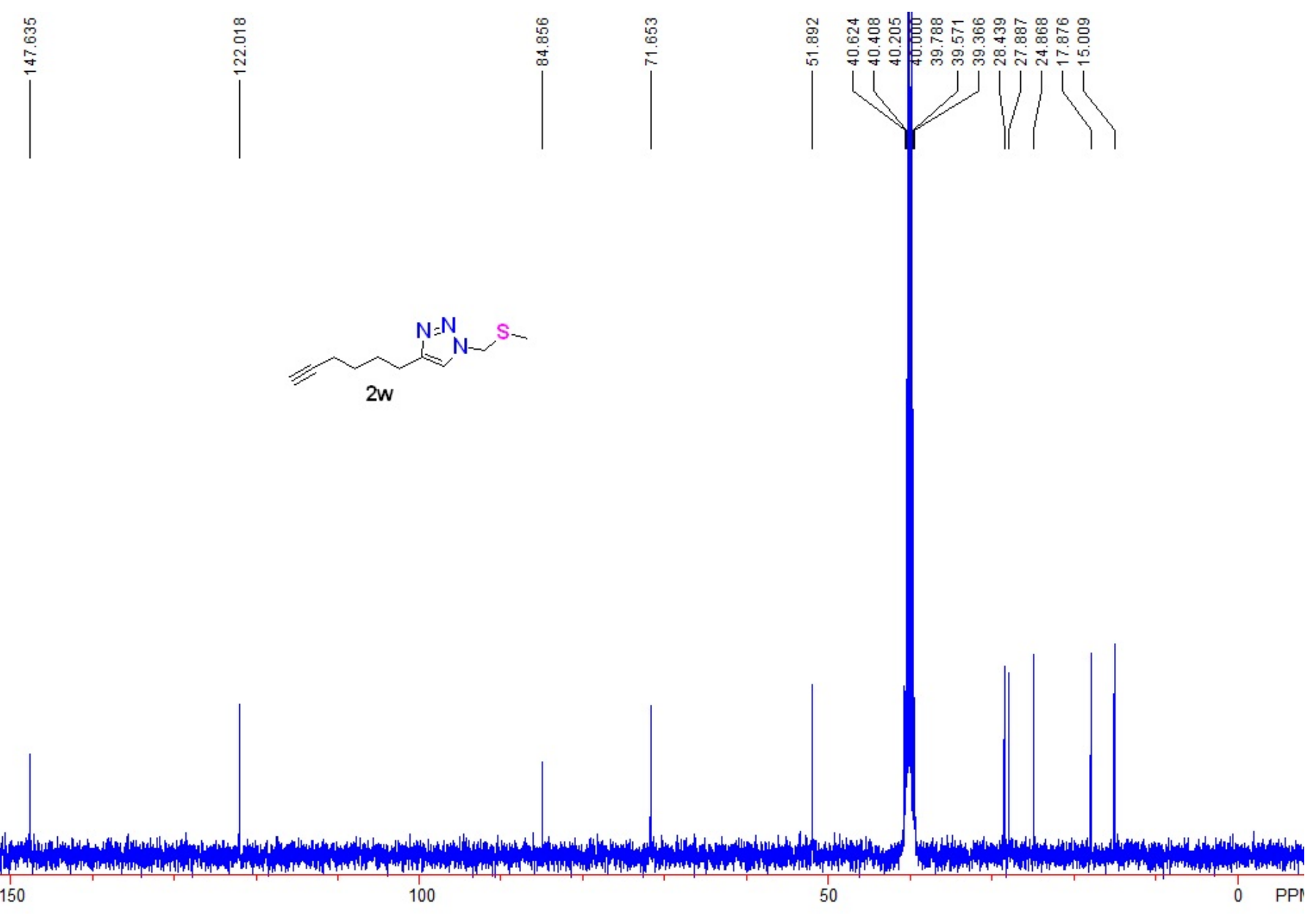



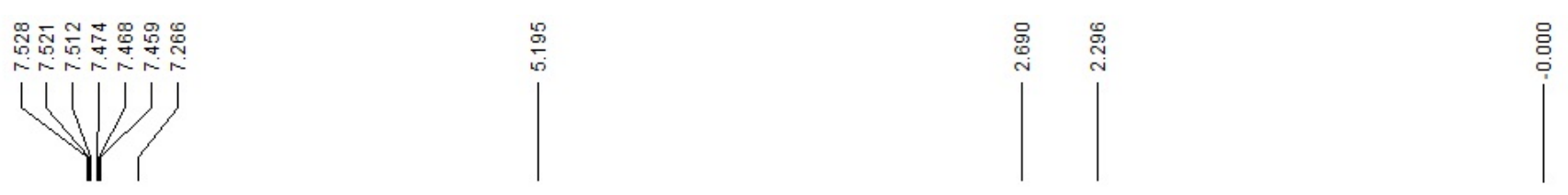

$$
\overbrace{2 x}^{P h}
$$

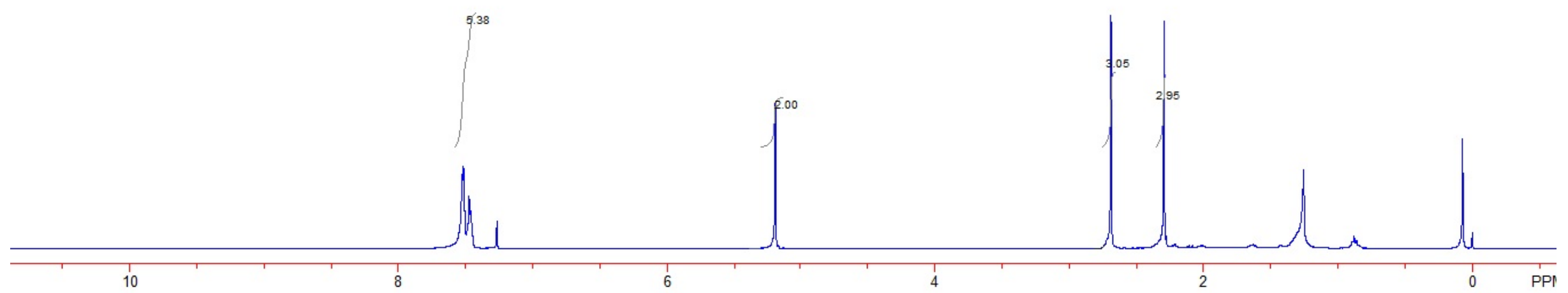



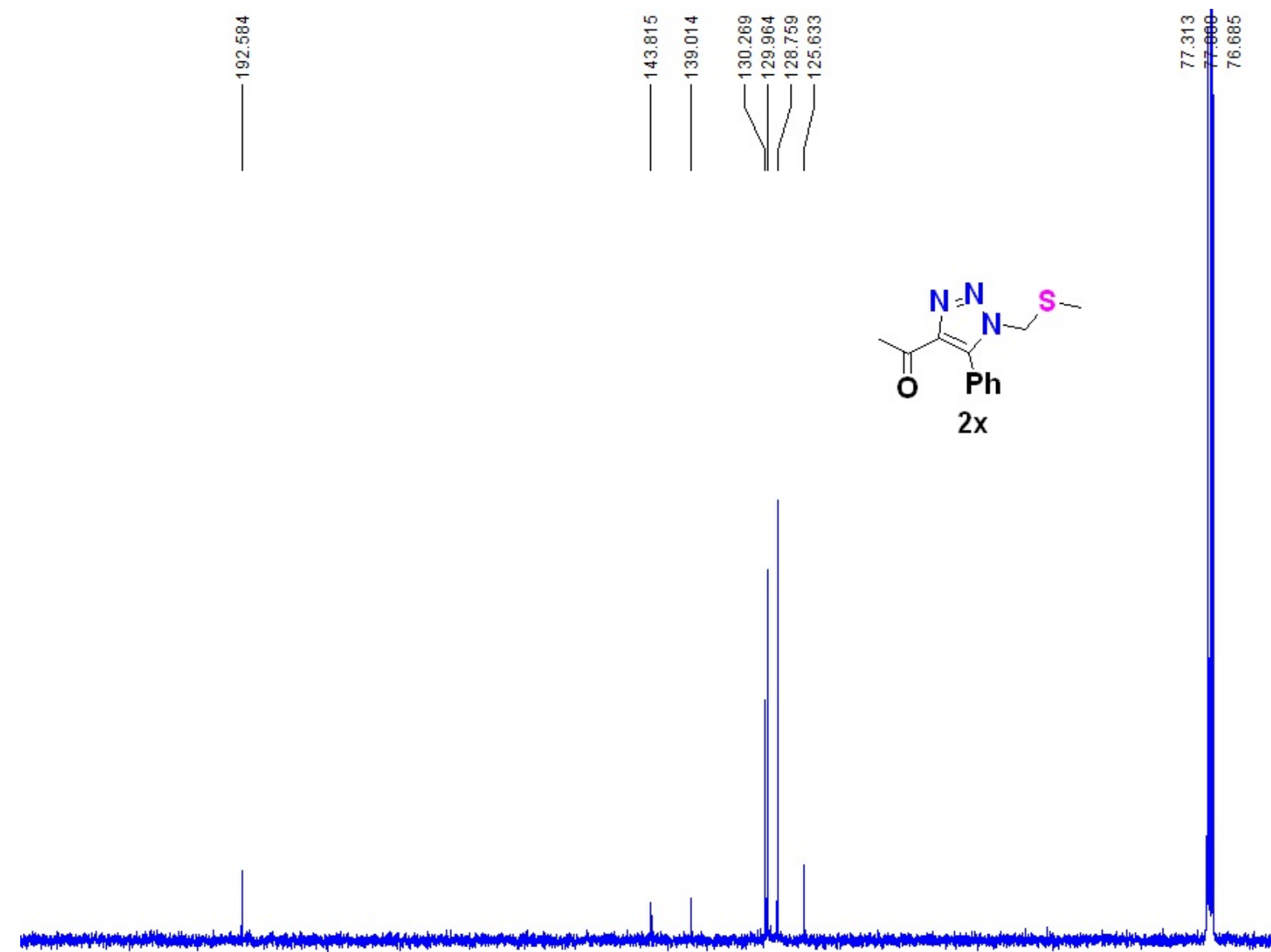


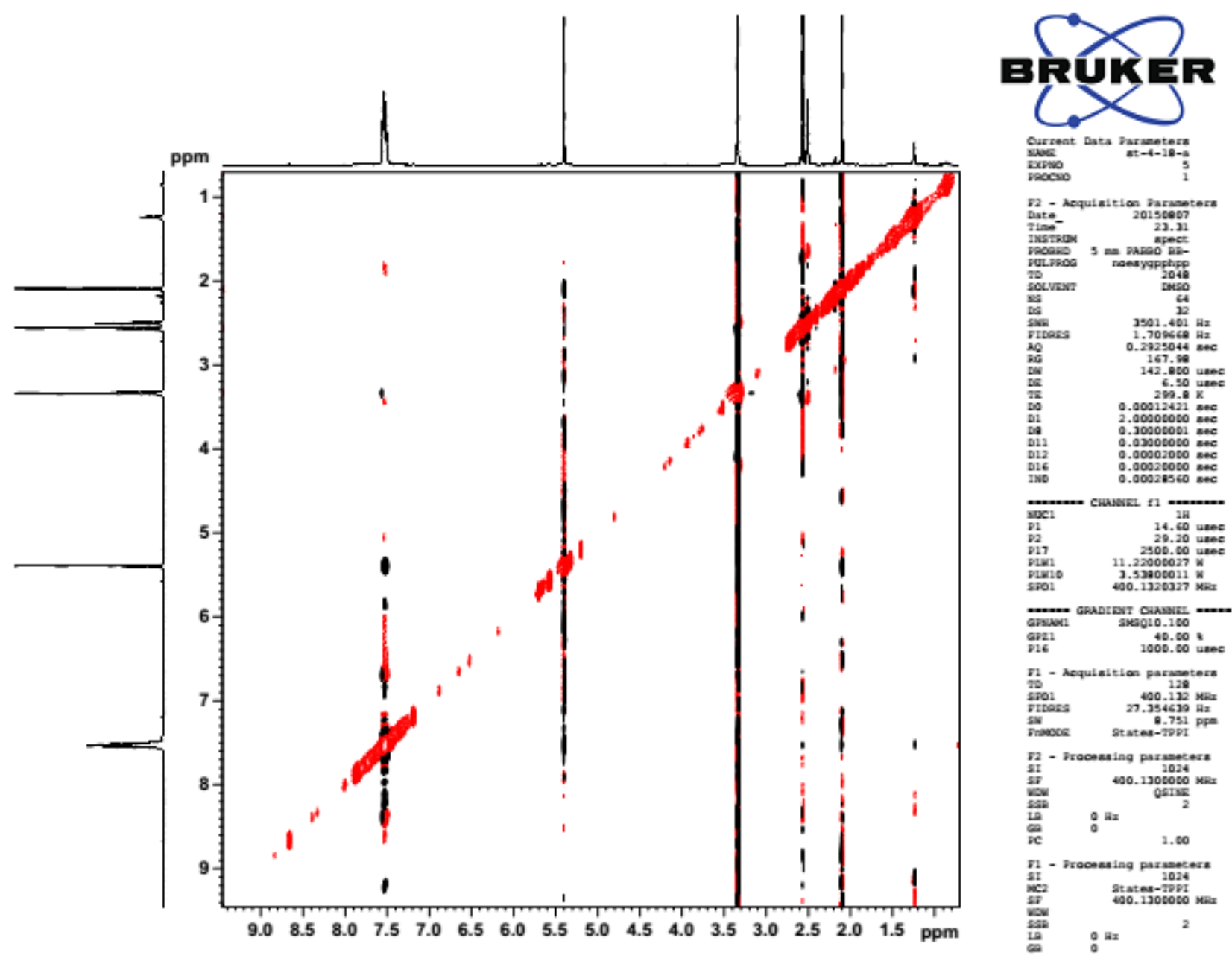

NOESY spectroscopy of $\mathbf{2} \mathbf{x}$ 


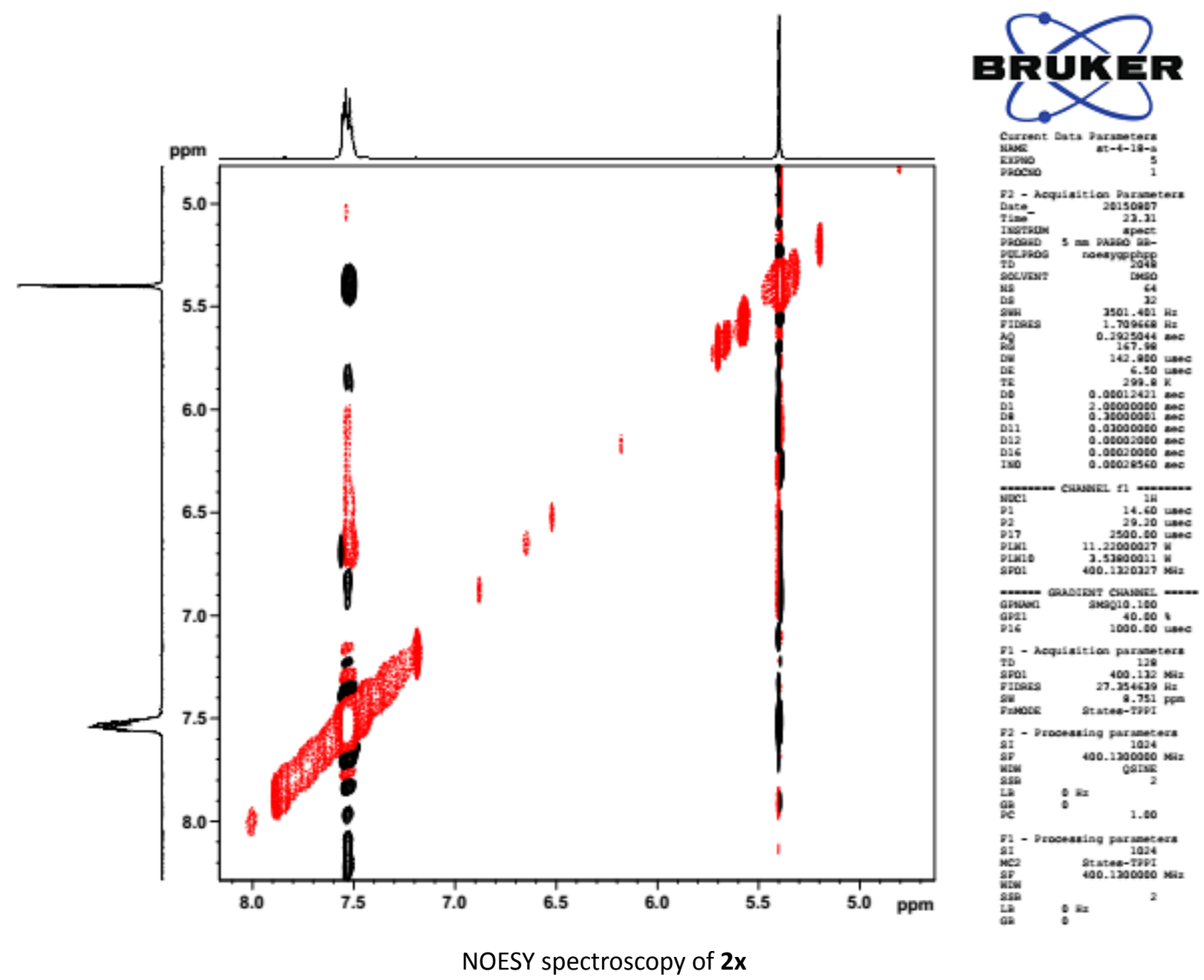



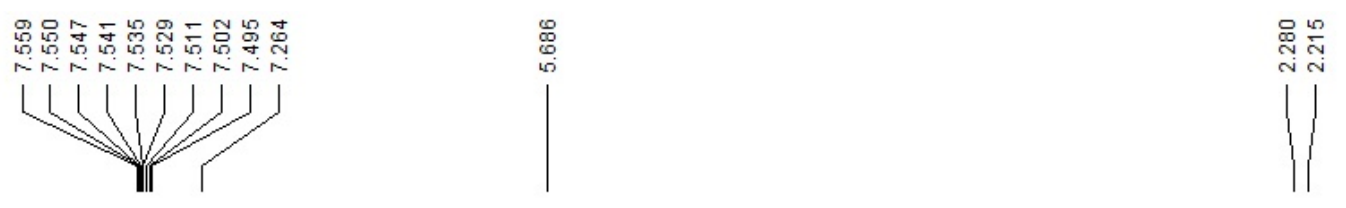

$$
\sum_{2 x^{\prime}}
$$

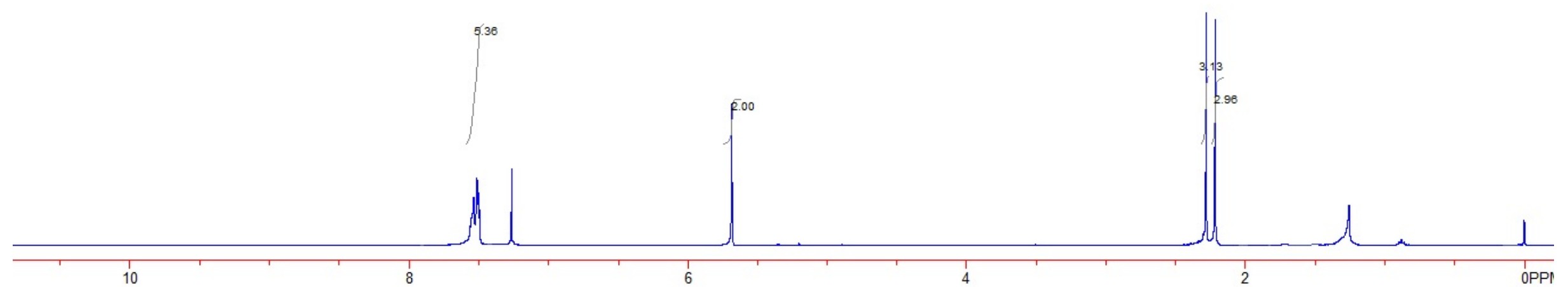



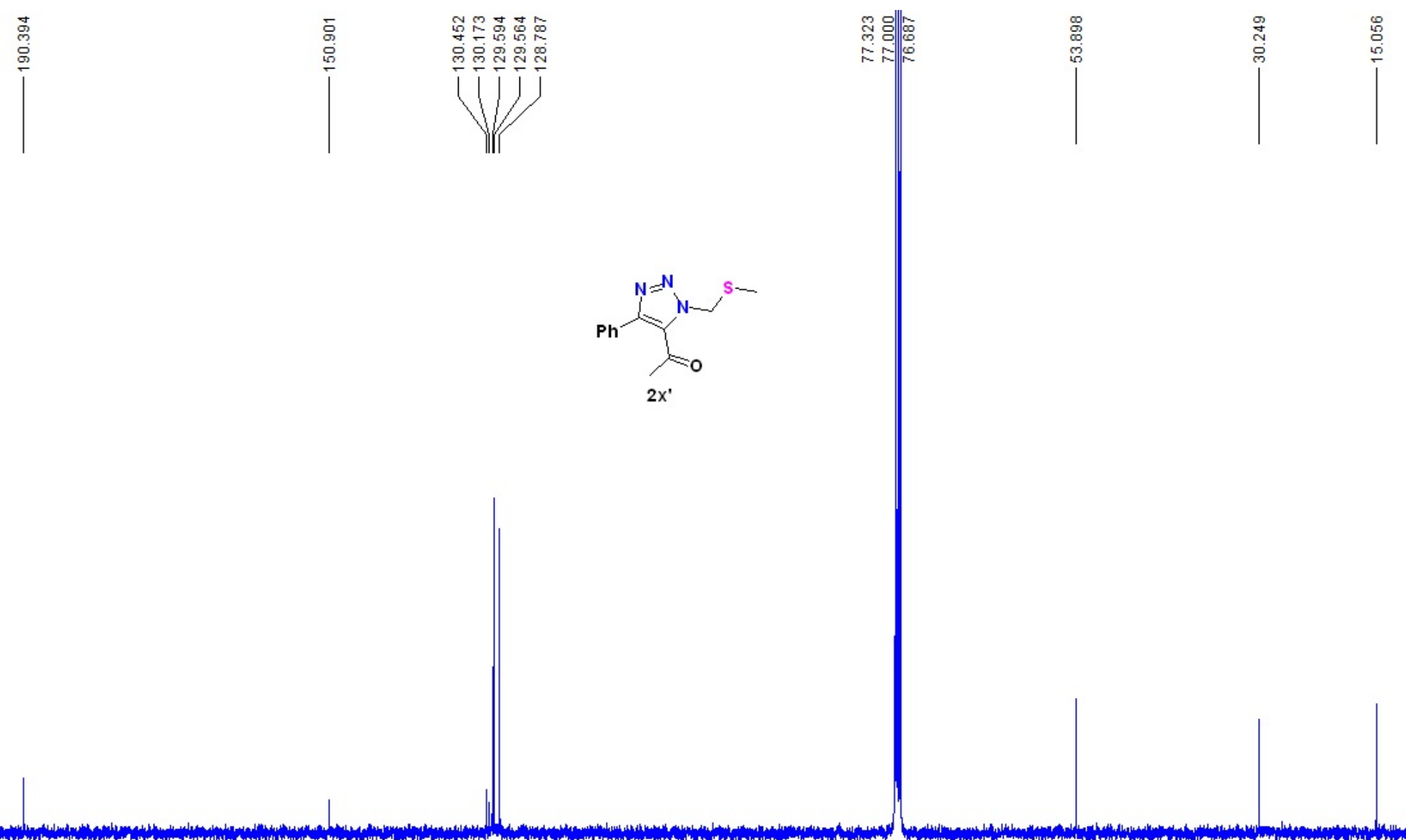


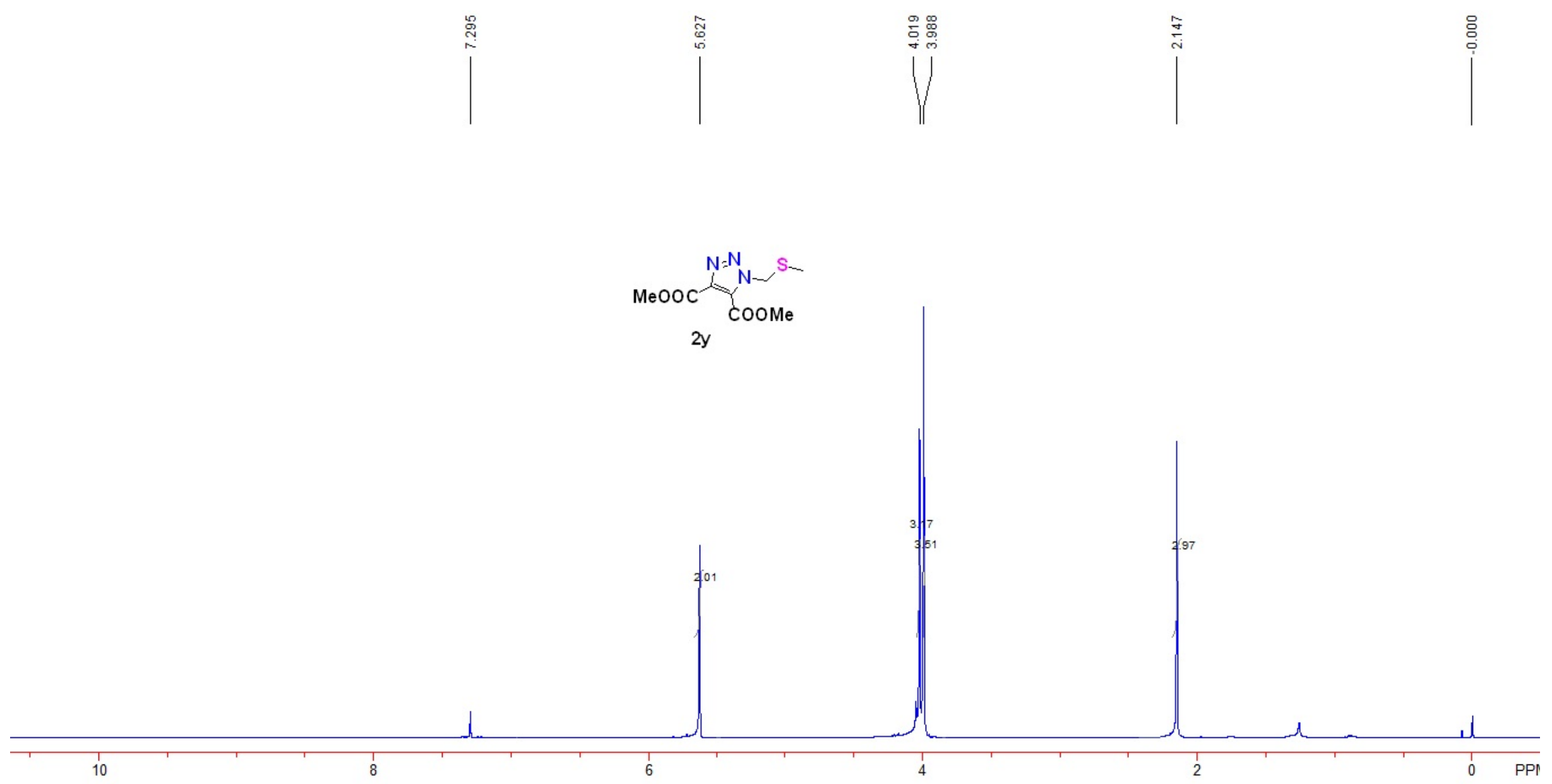



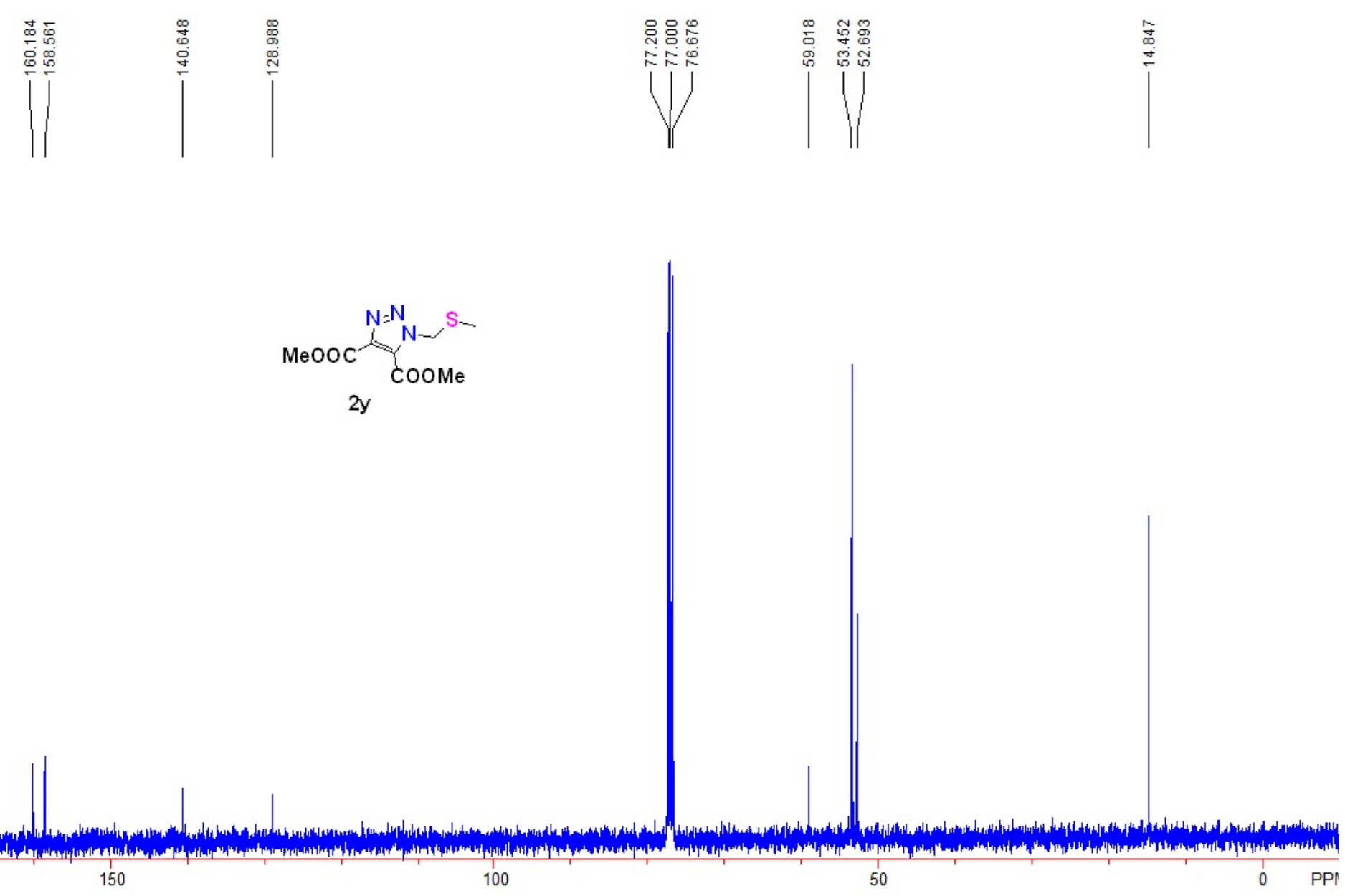
我总总品

il

总
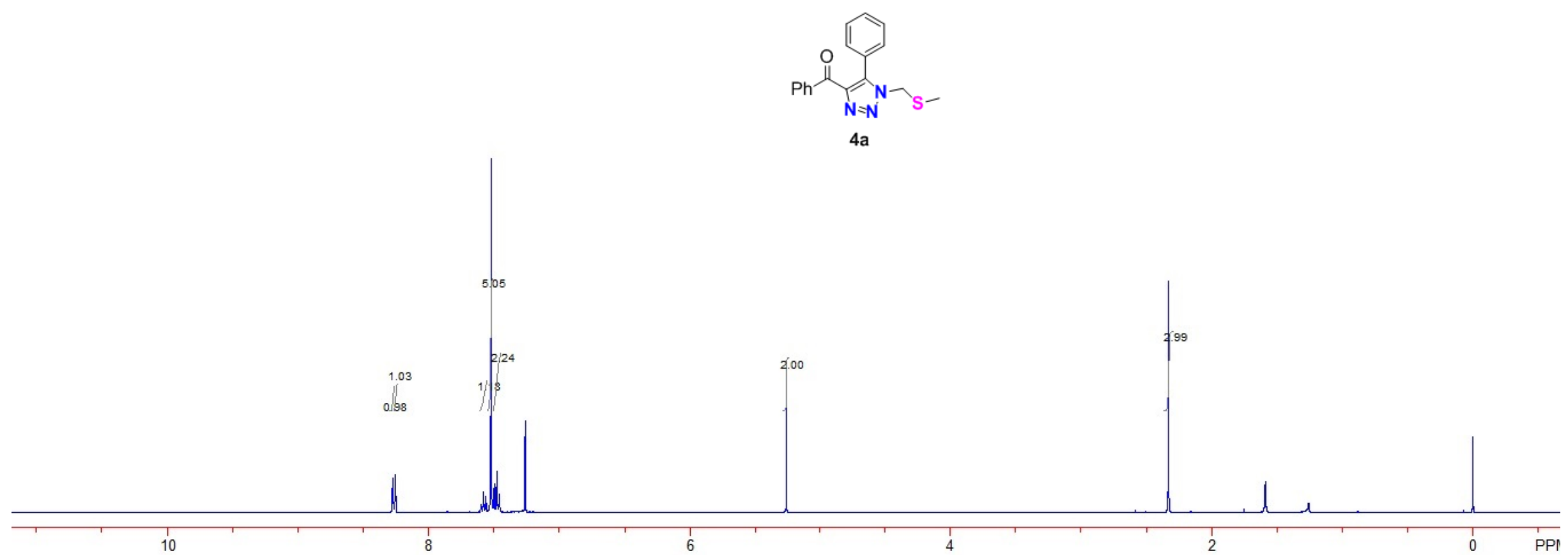

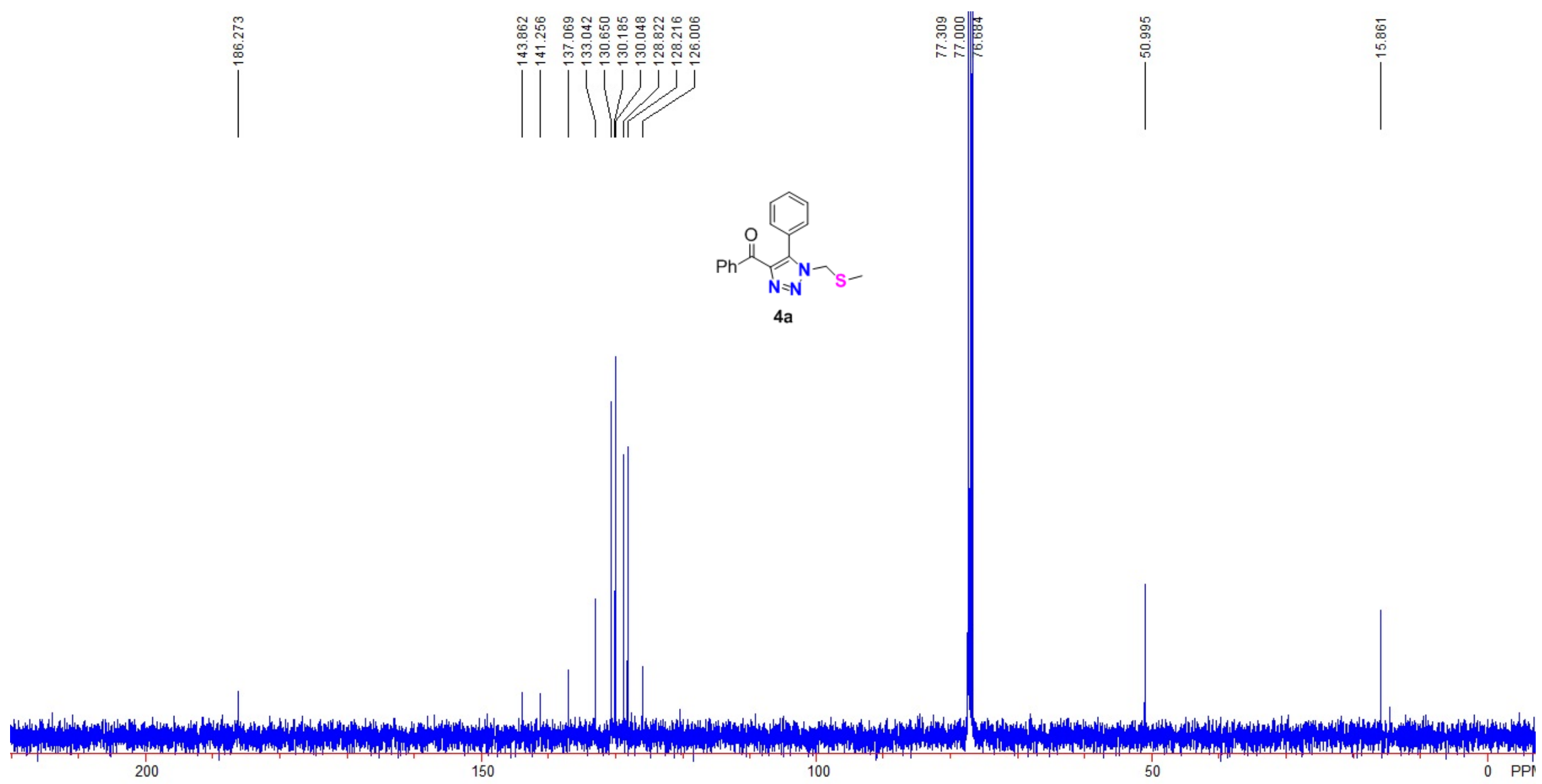


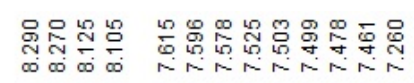

ul

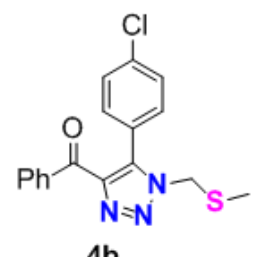

$4 \mathrm{~b}$

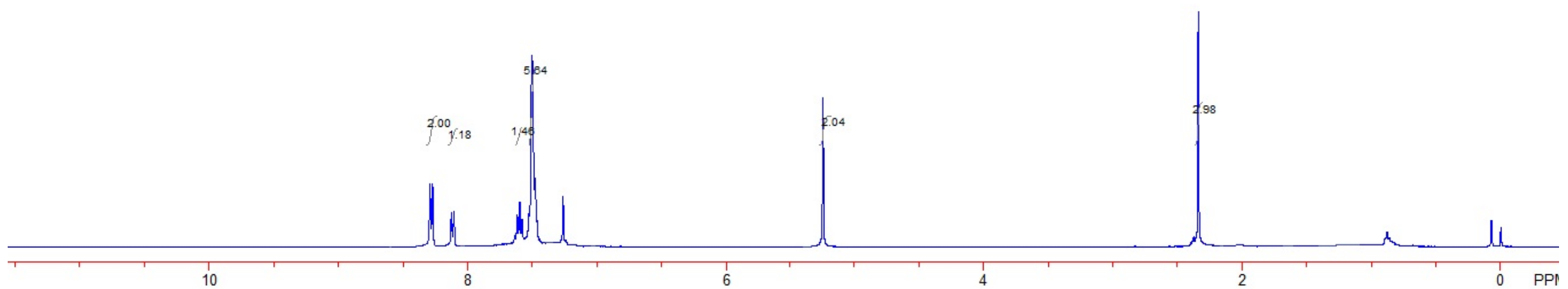



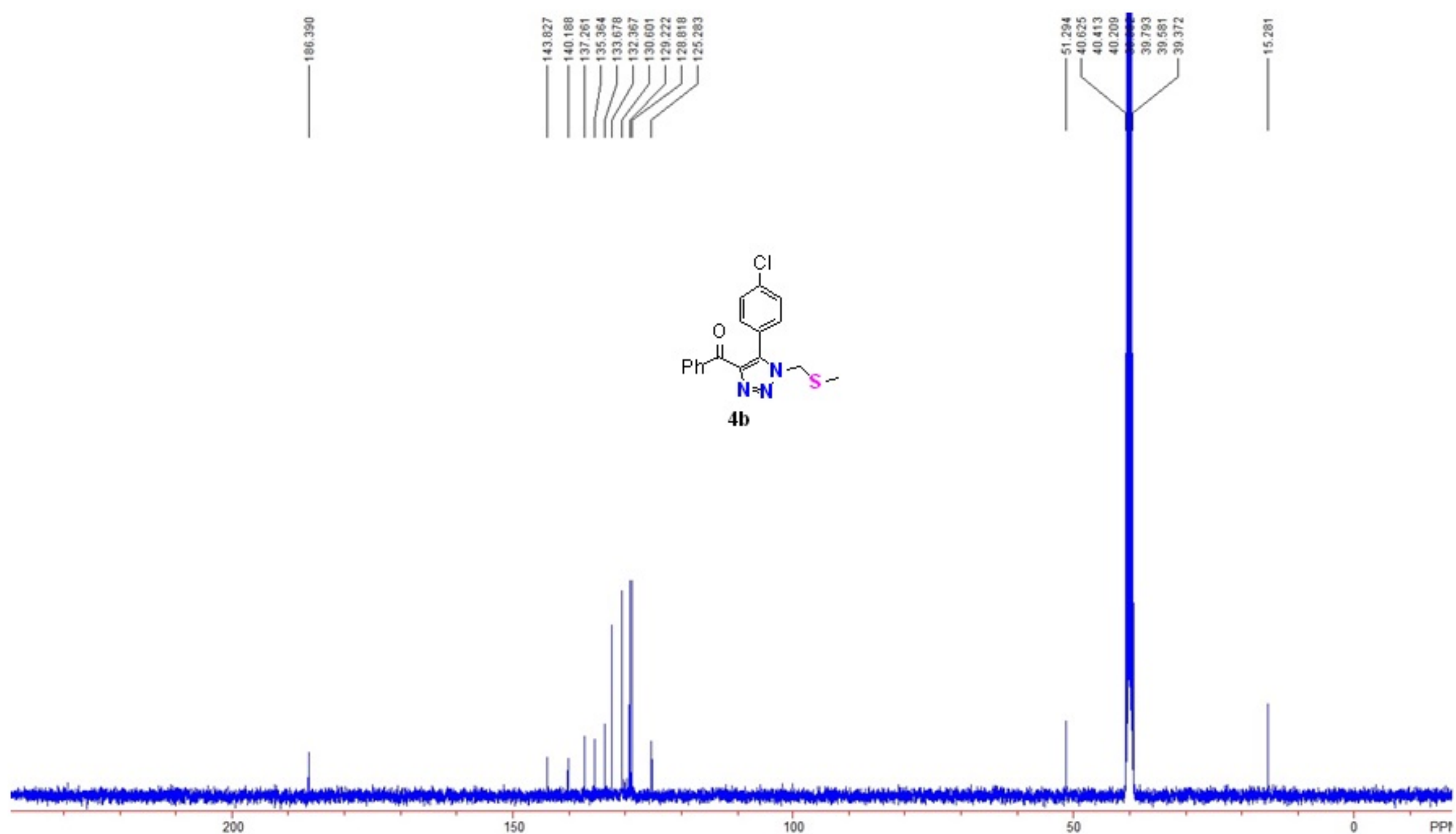

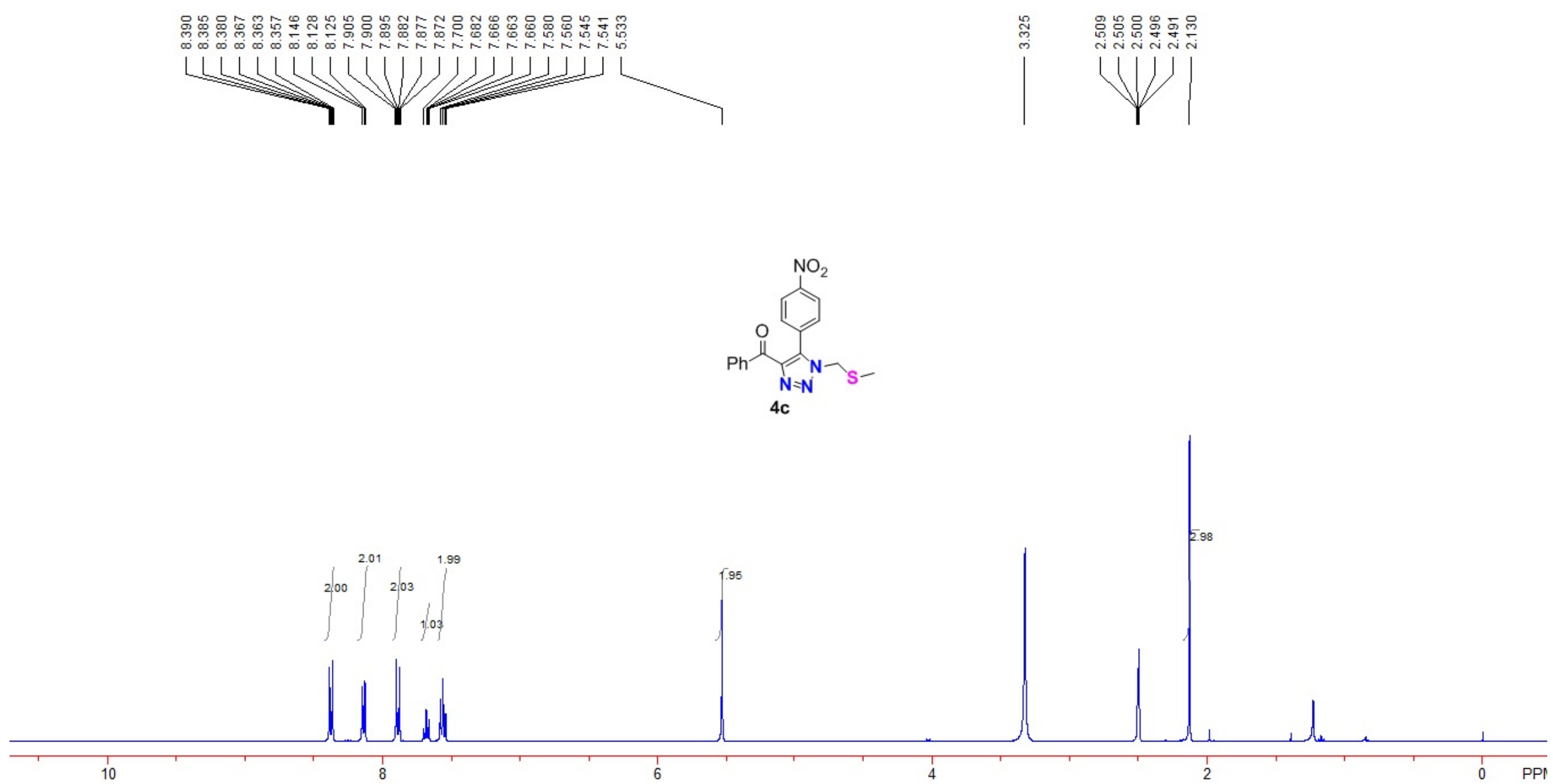

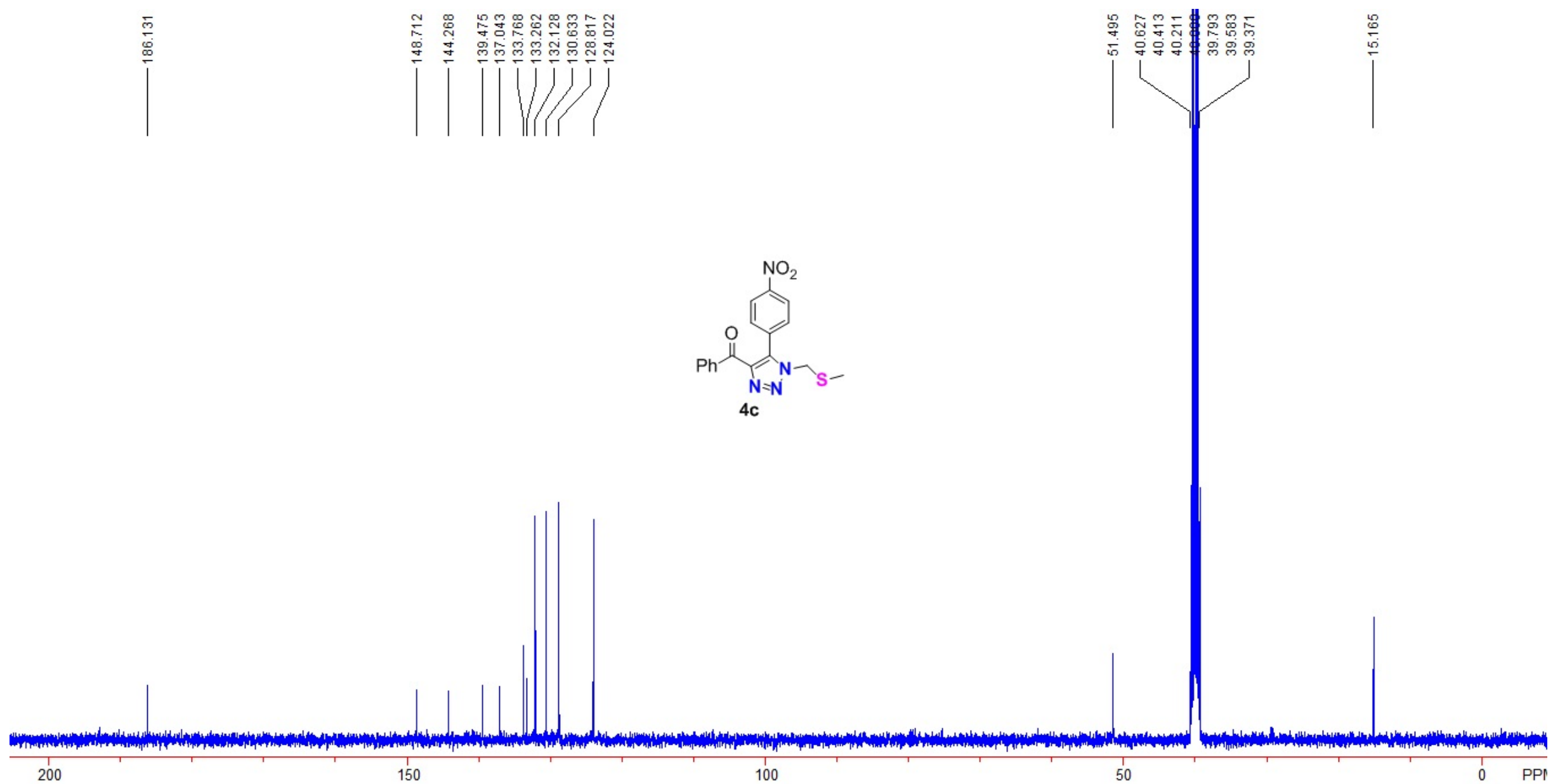


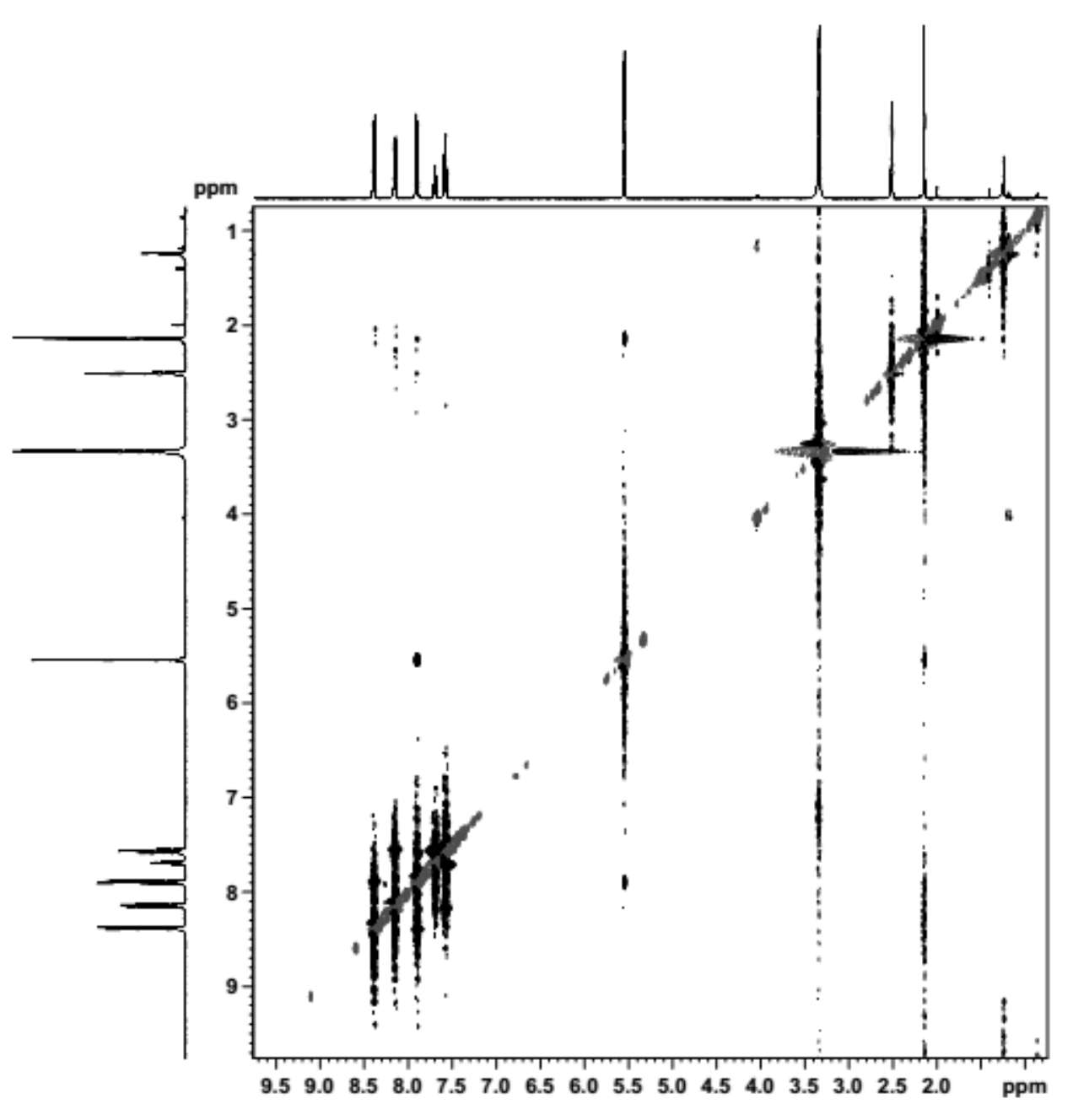

\section{BRUKER}

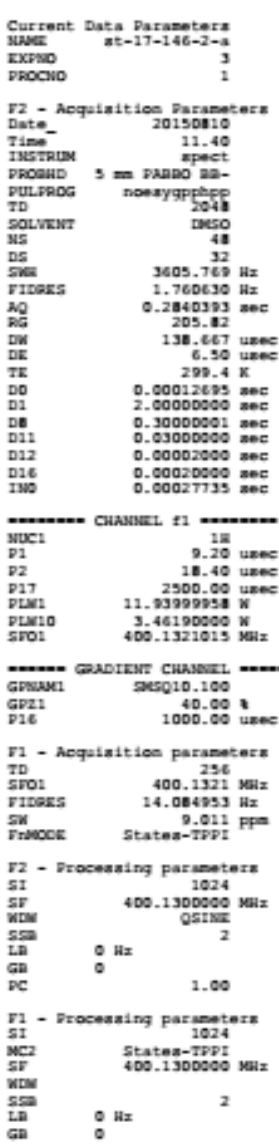

NOESY spectroscopy of $4 c$ 


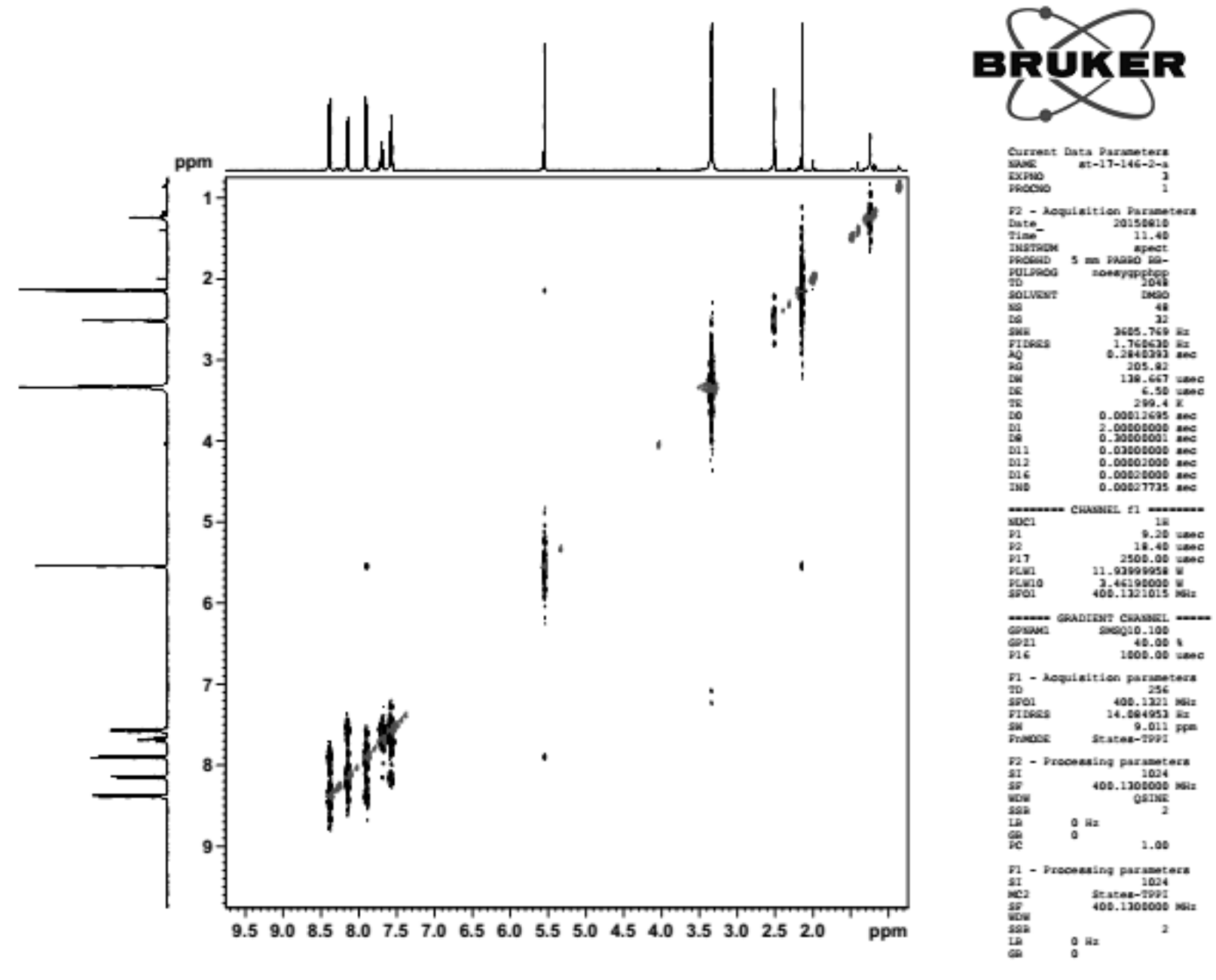

NOESY spectroscopy of $\mathbf{4 c}$ 

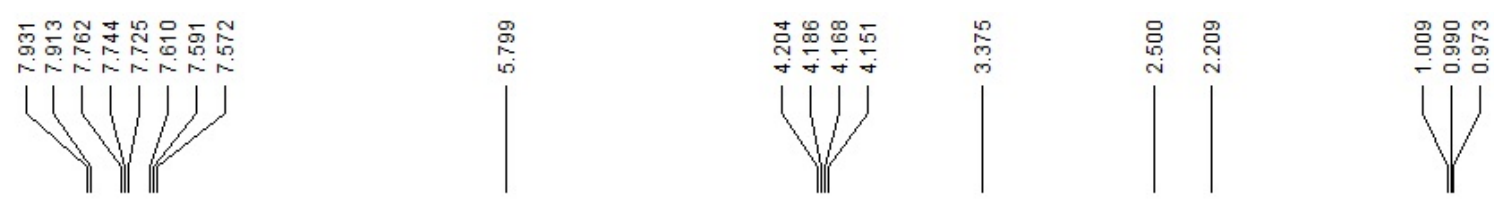

$$
\underbrace{\mathrm{N}}_{\substack{\mathrm{N}=\mathrm{N} \\ 4 d}}
$$

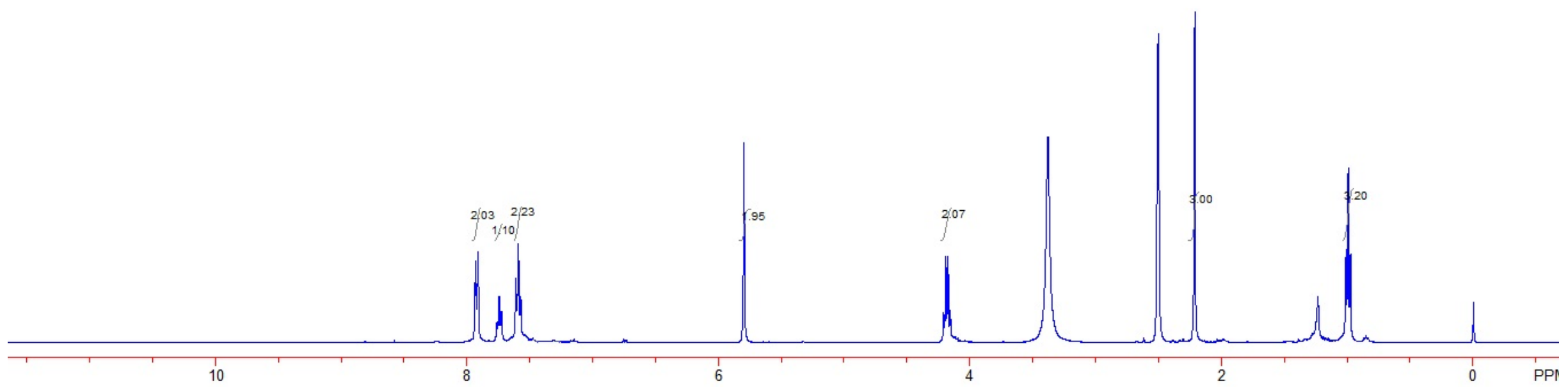



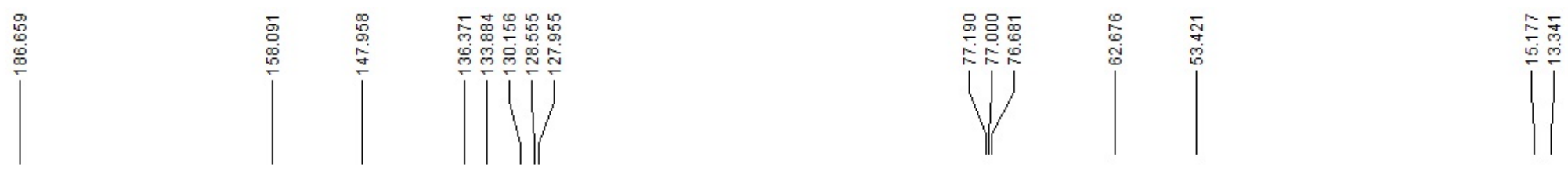

$$
\underbrace{N-}_{\substack{N=N \\ 4 d}}
$$

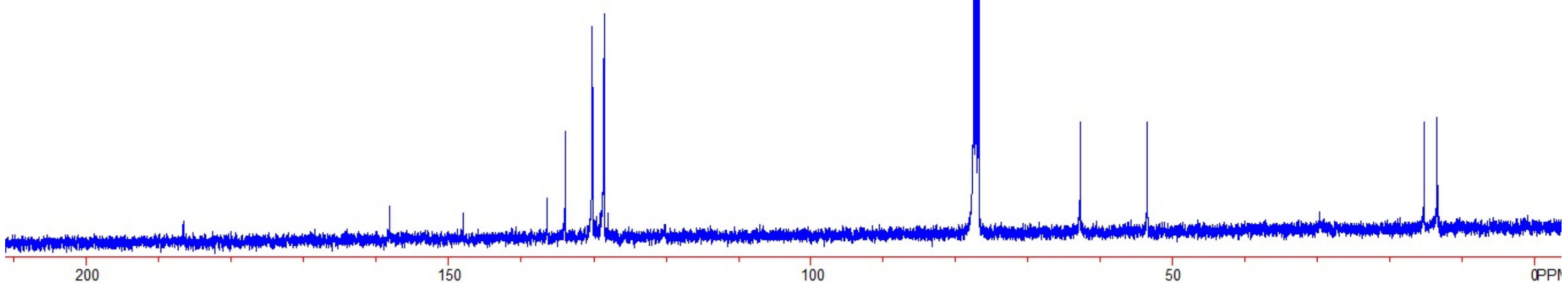



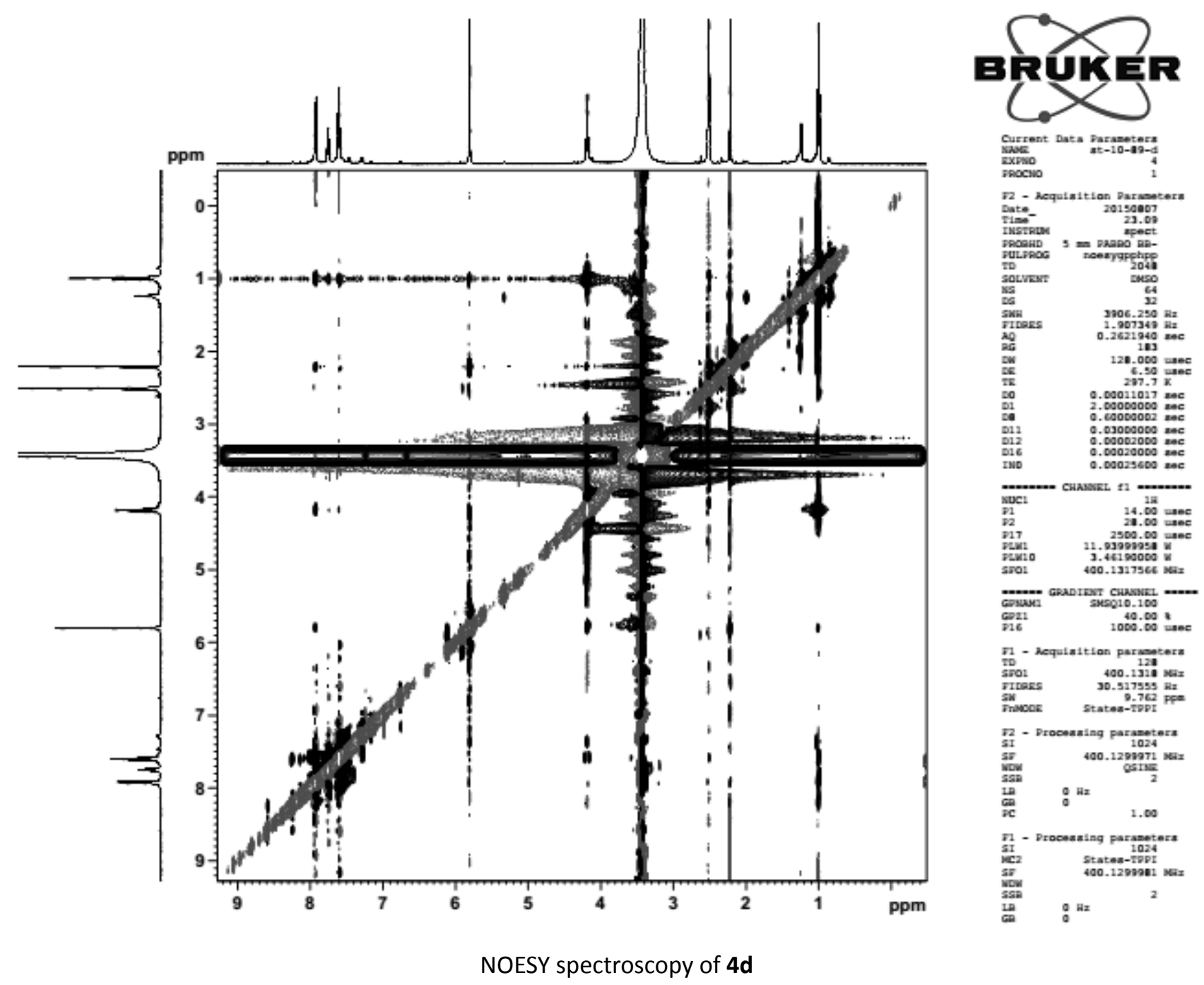

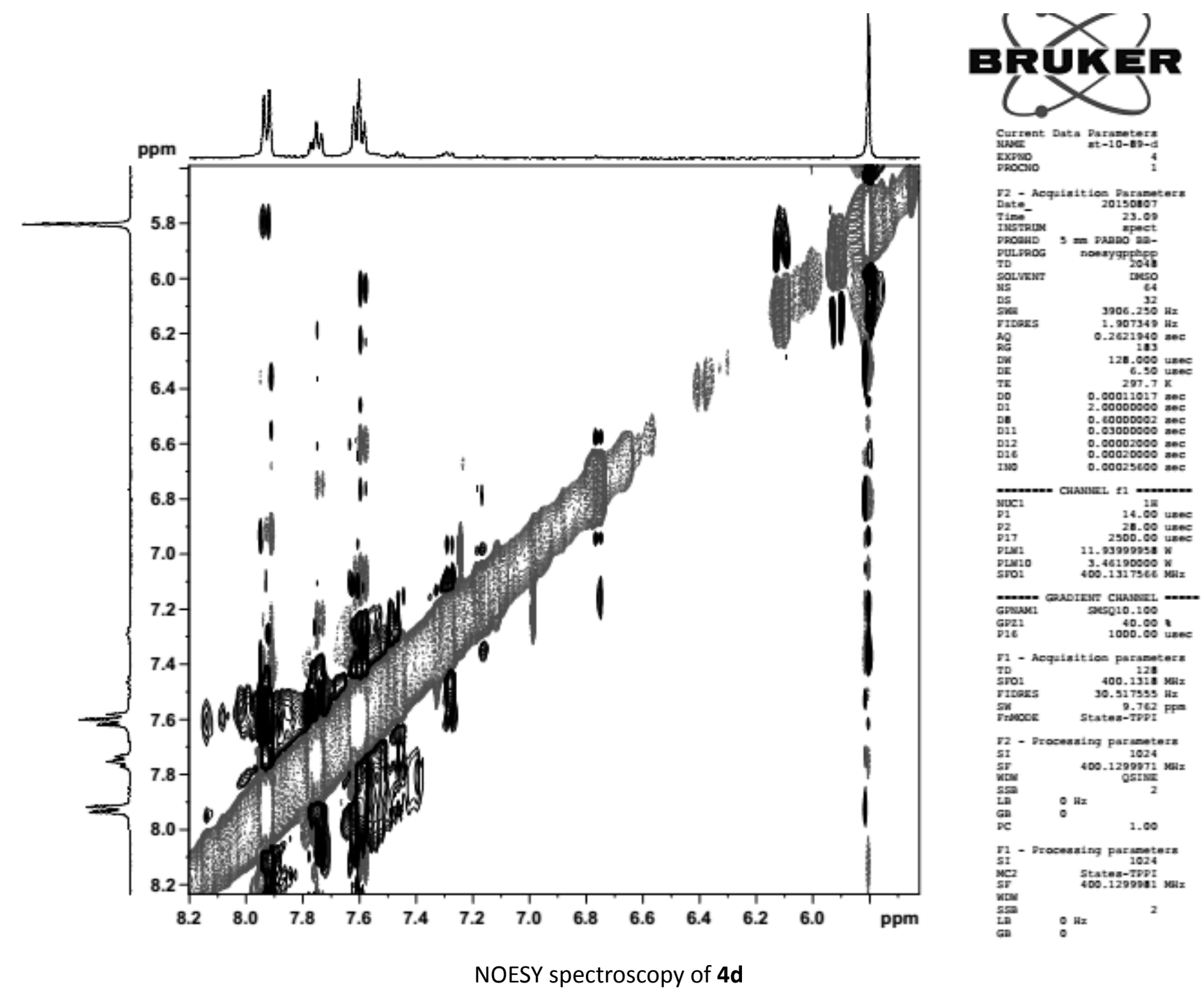

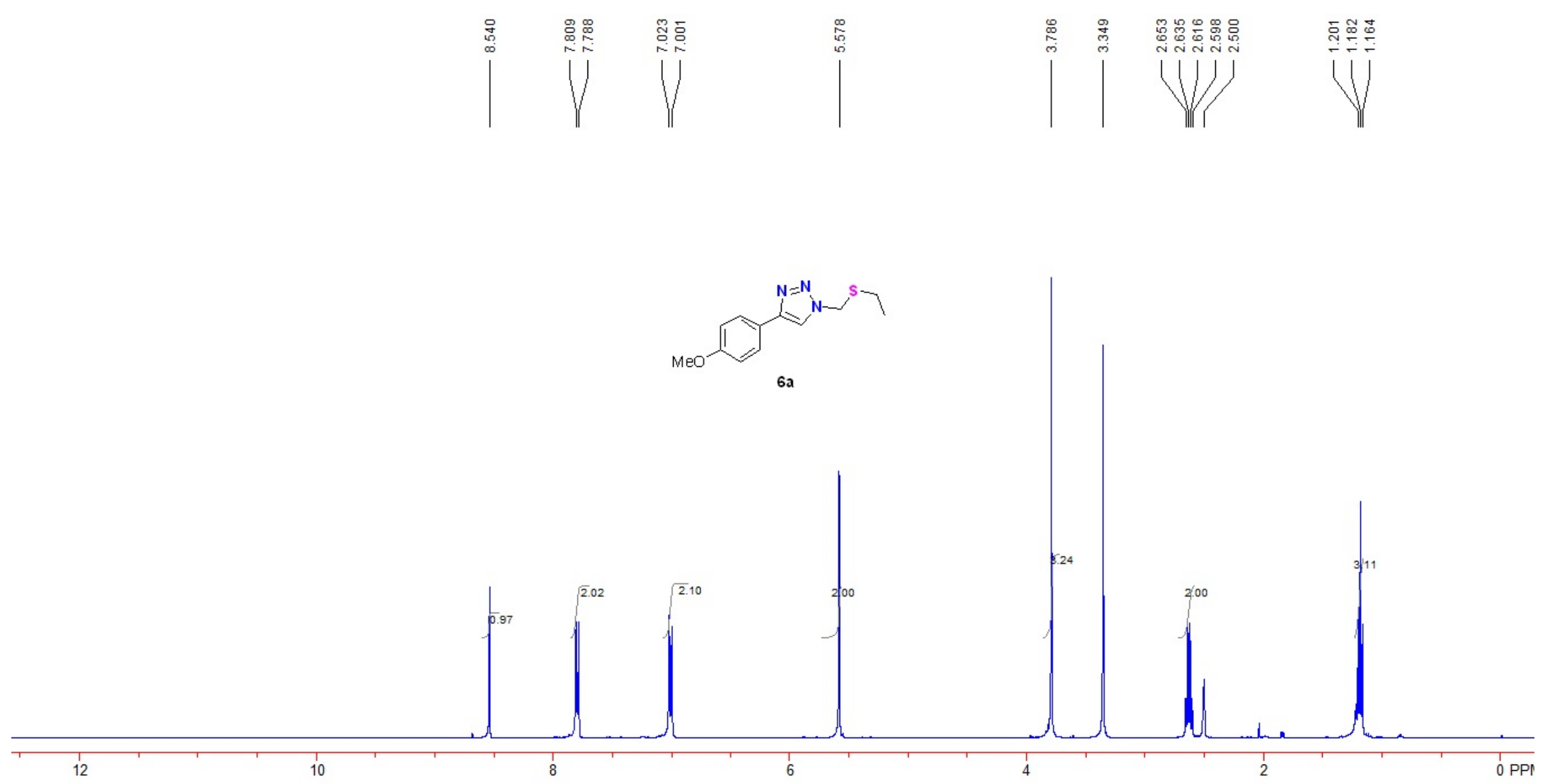


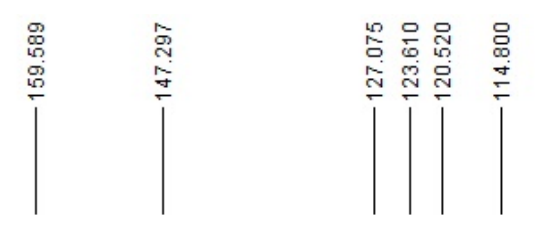

$\mid$

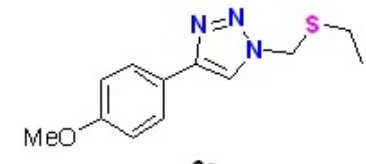

6

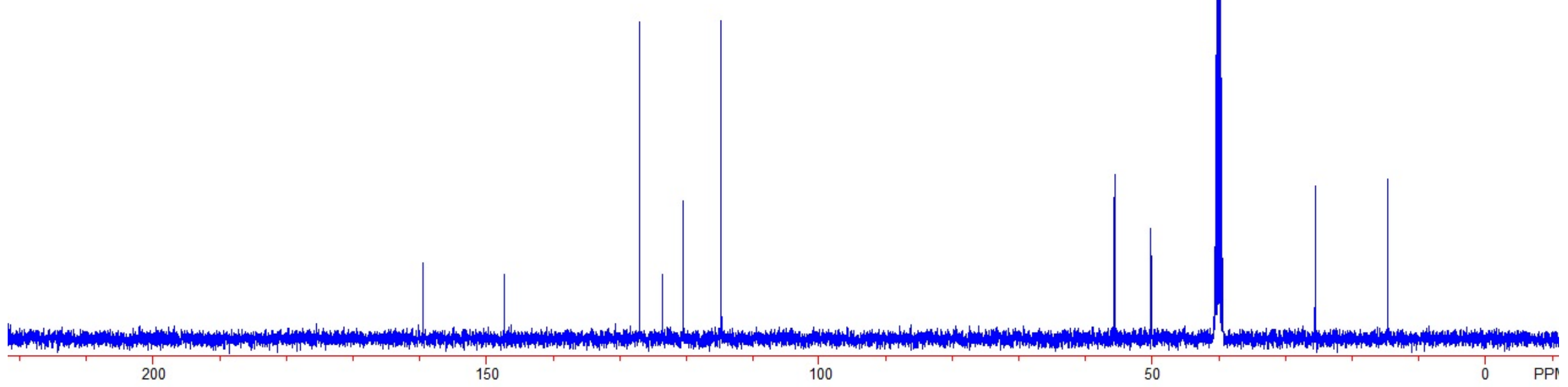




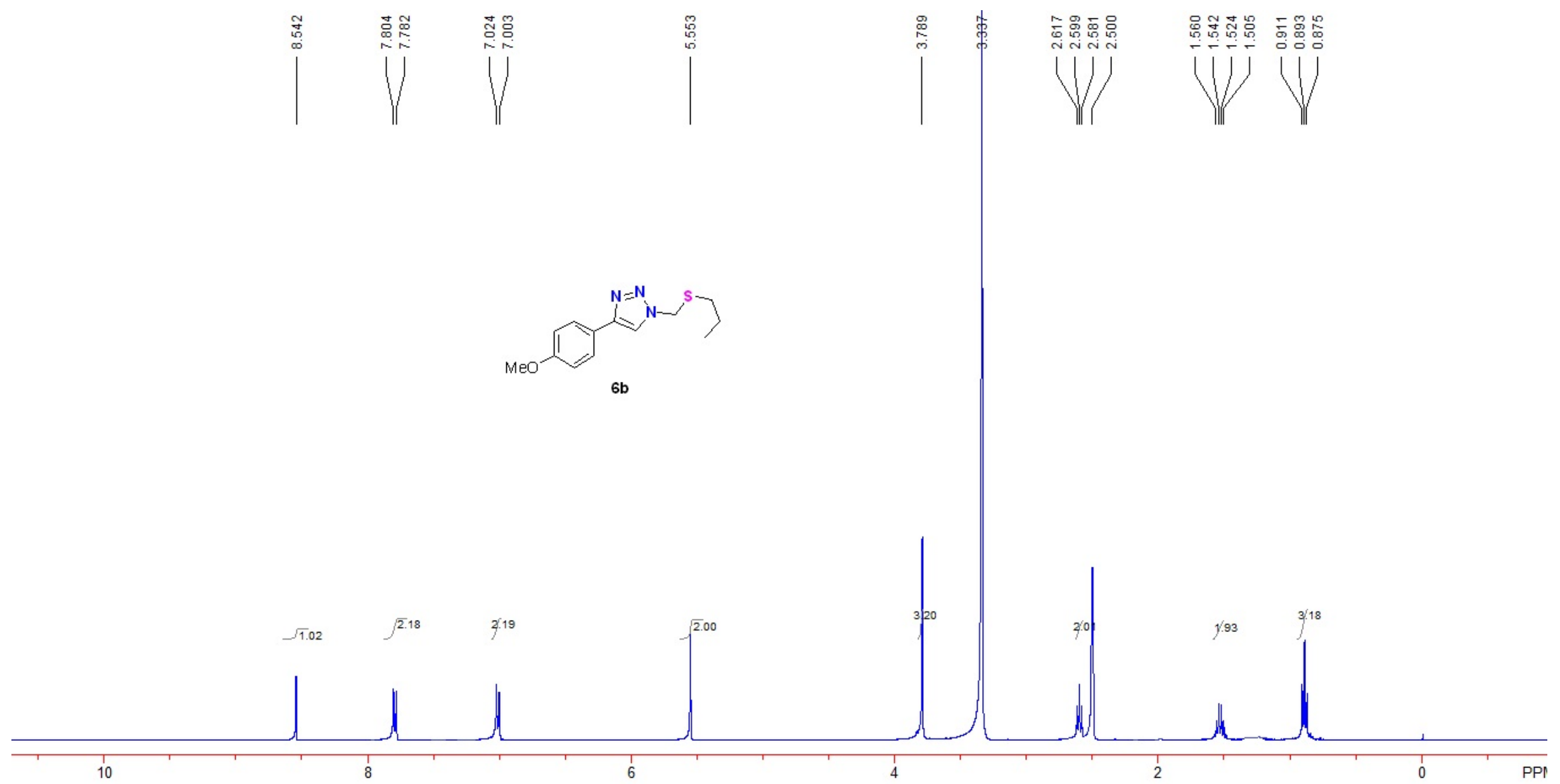




$\left.\right|^{\substack{0 \\ 0}}$

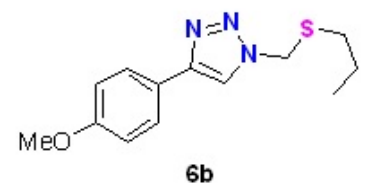

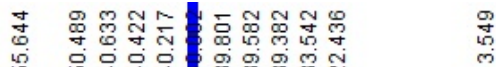
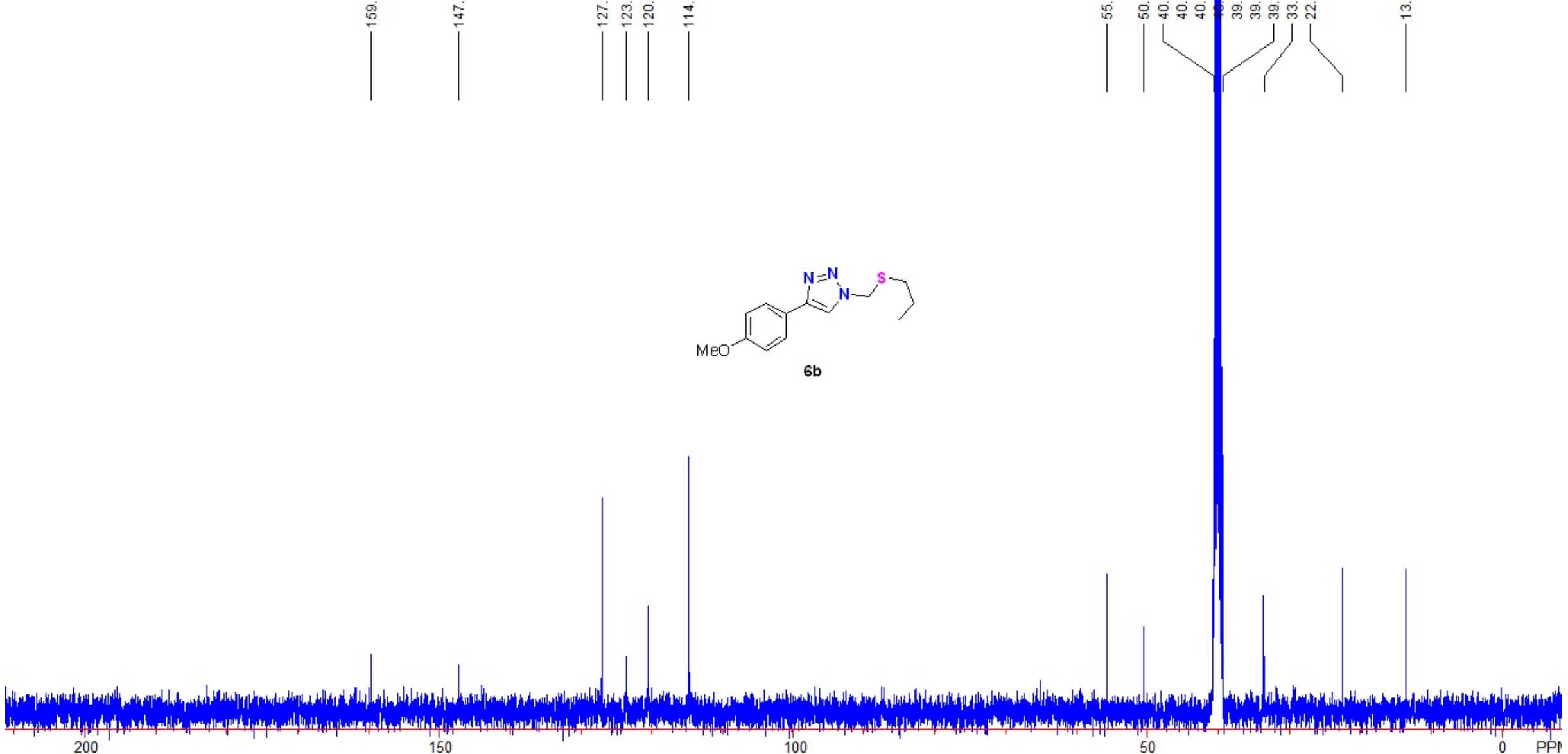

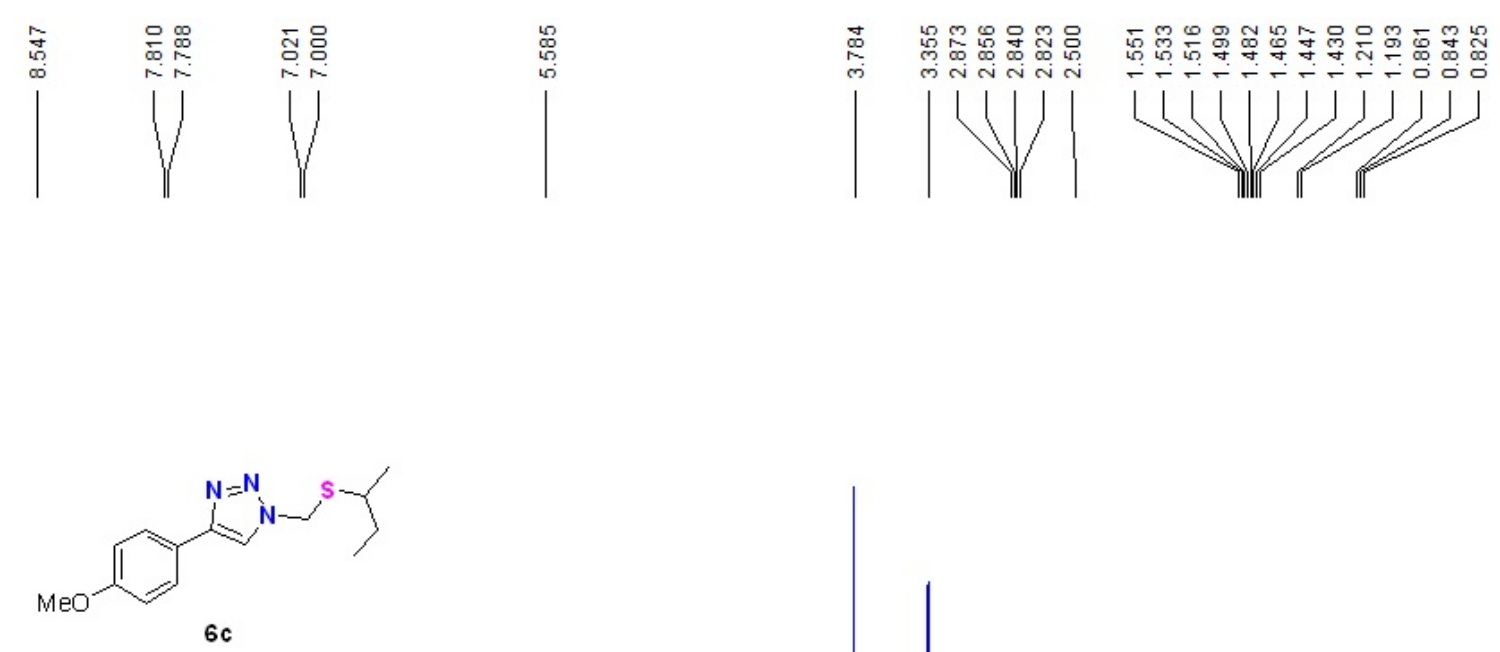

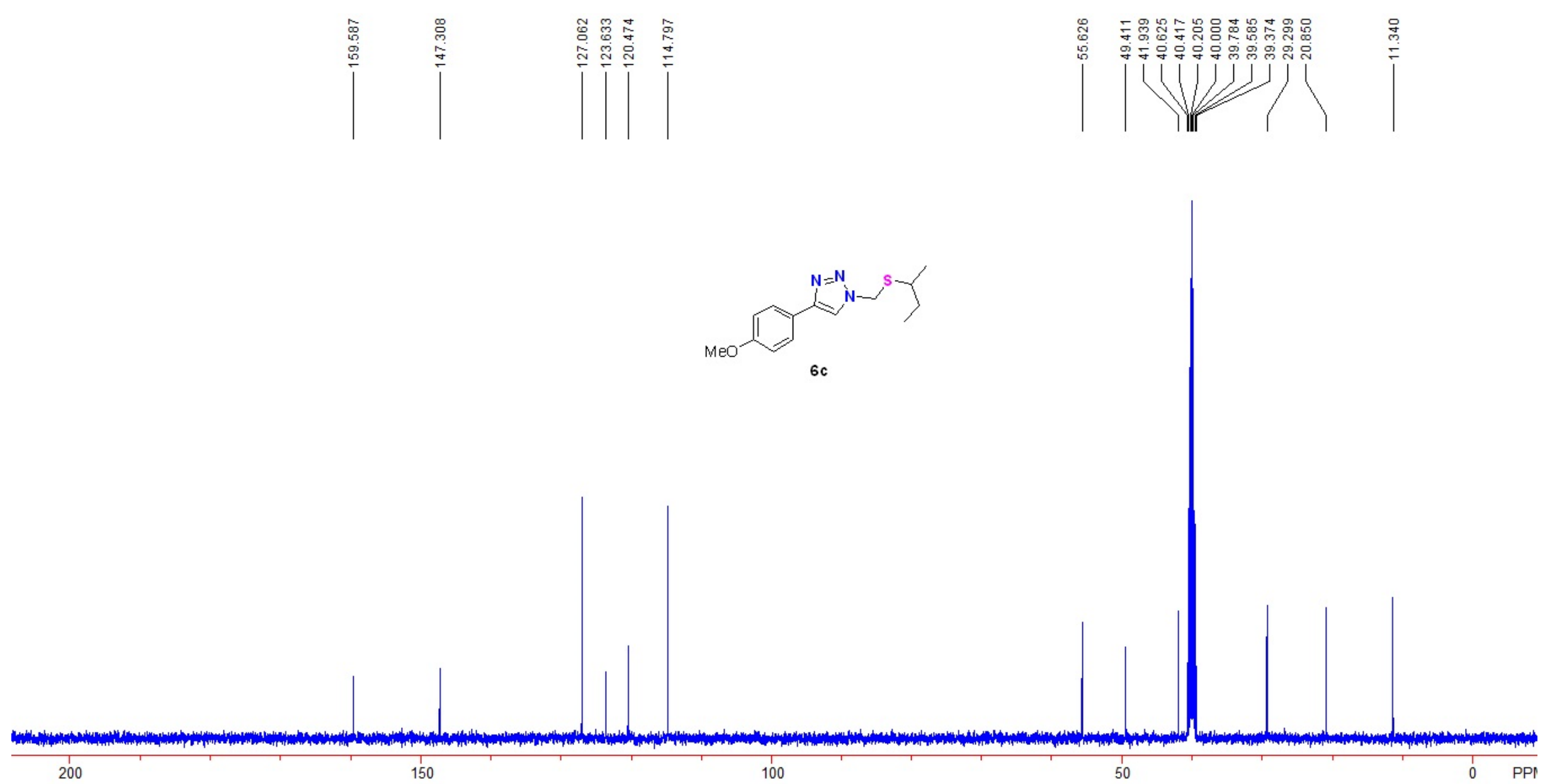


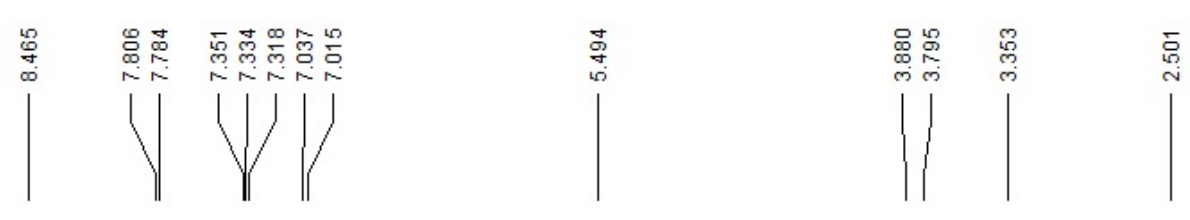
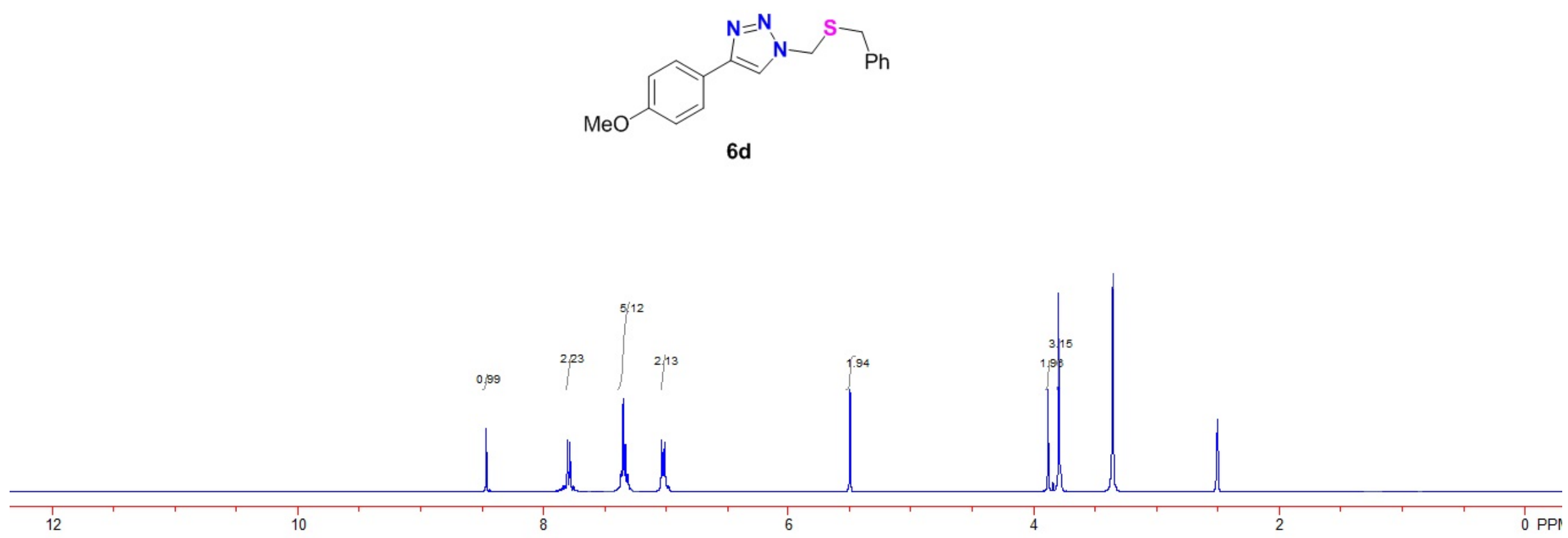

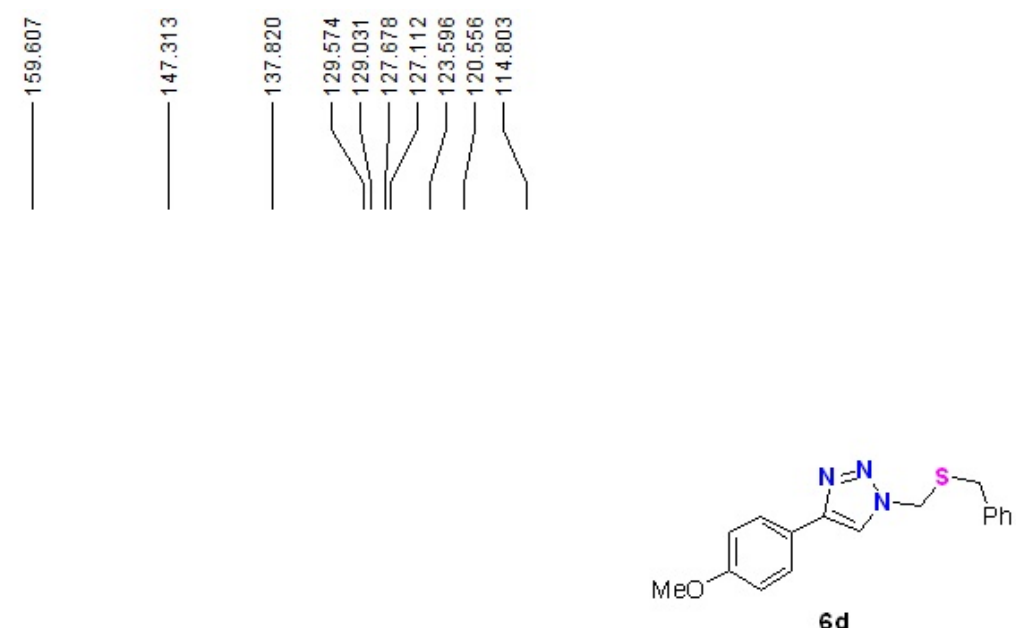

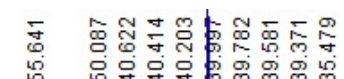

ใบำj

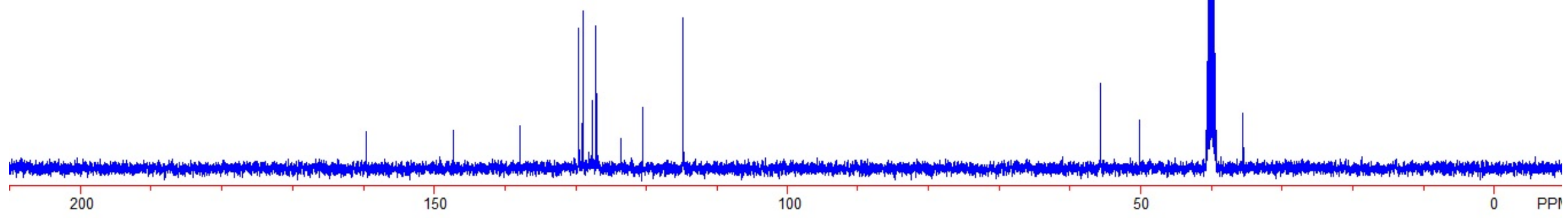




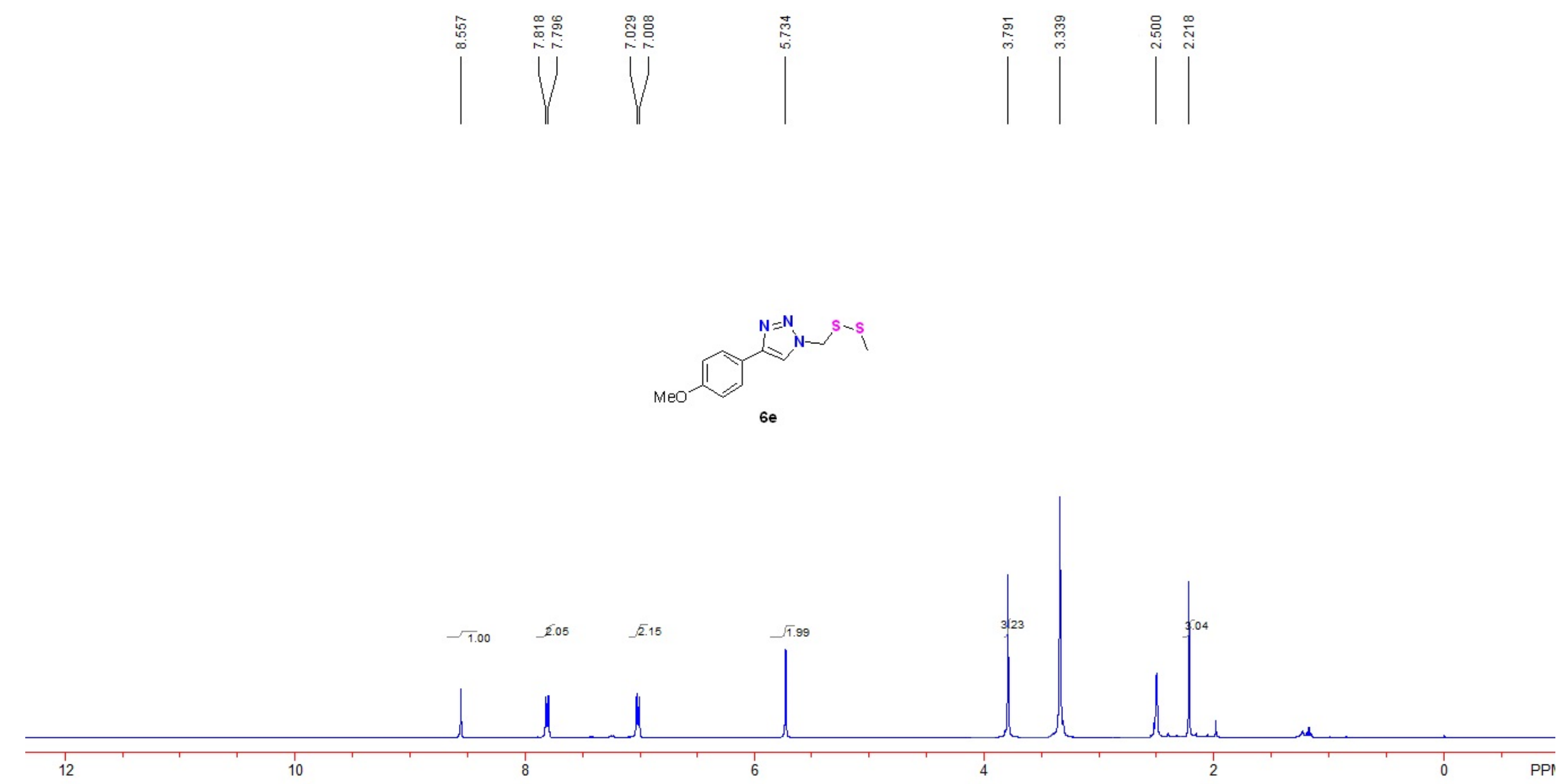




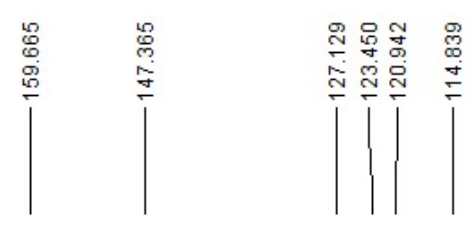

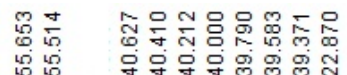

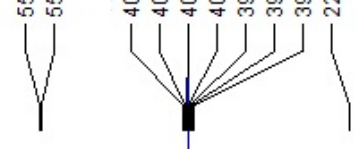

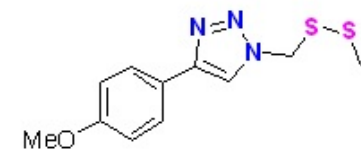

6e 

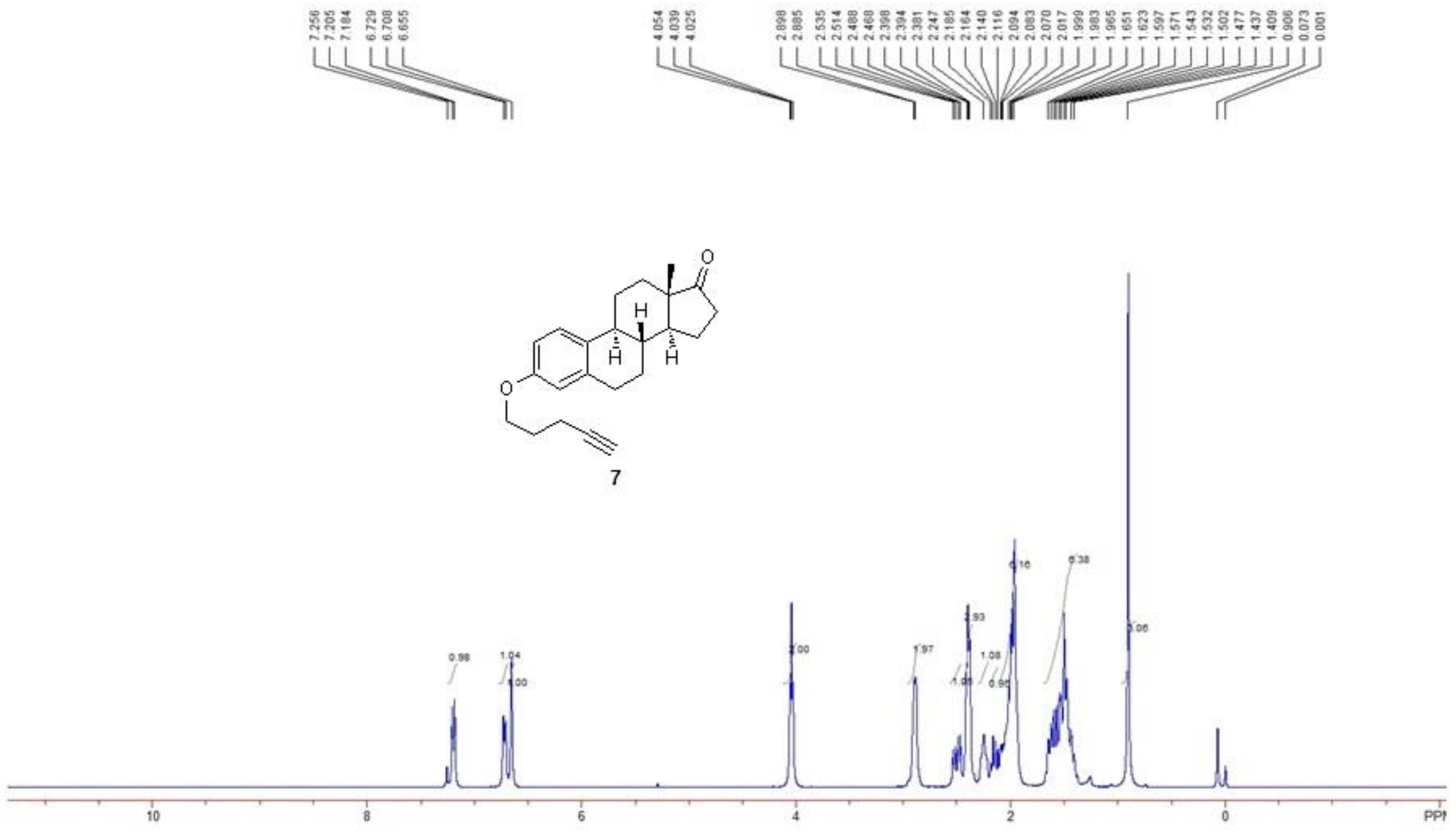

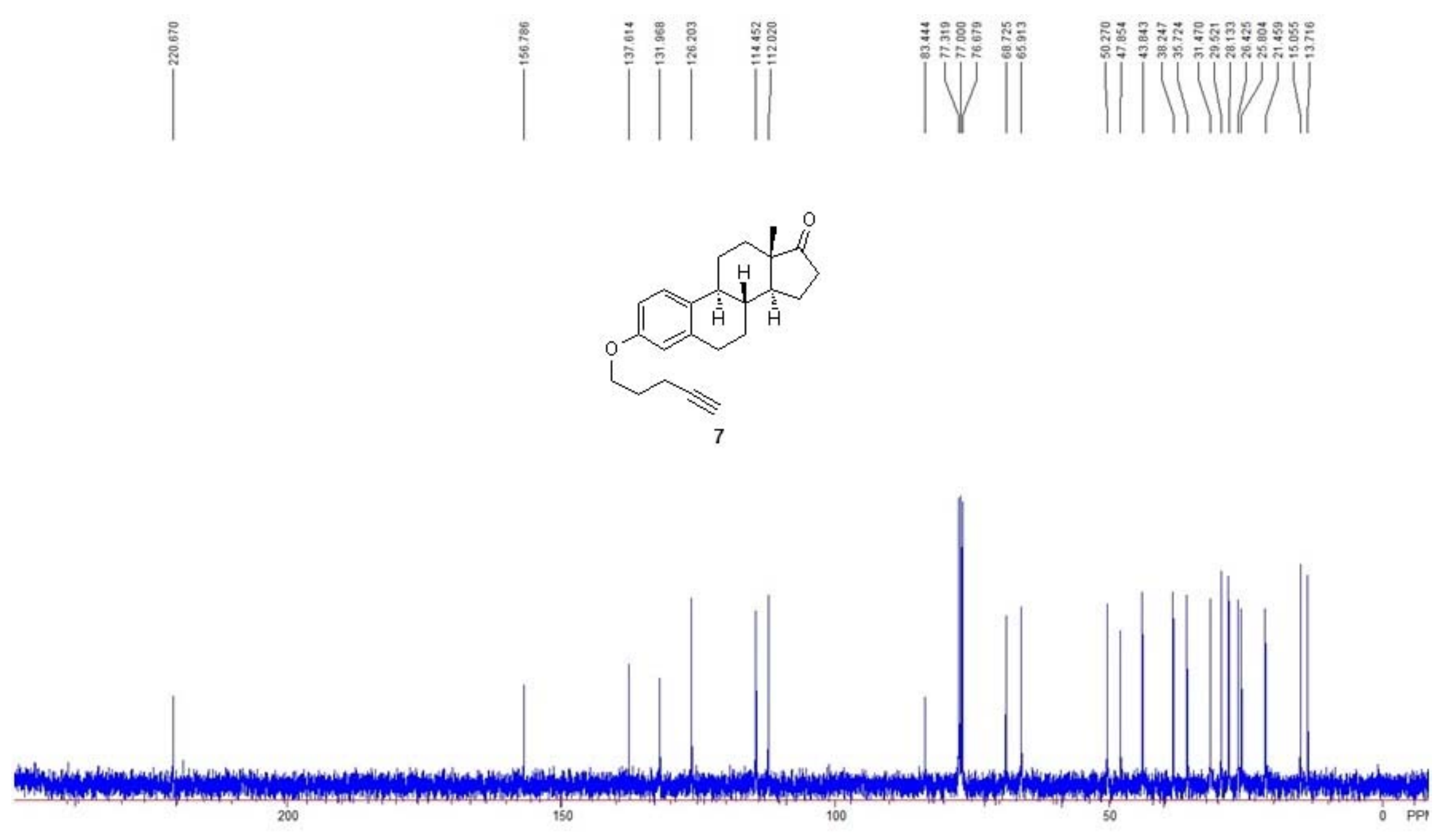


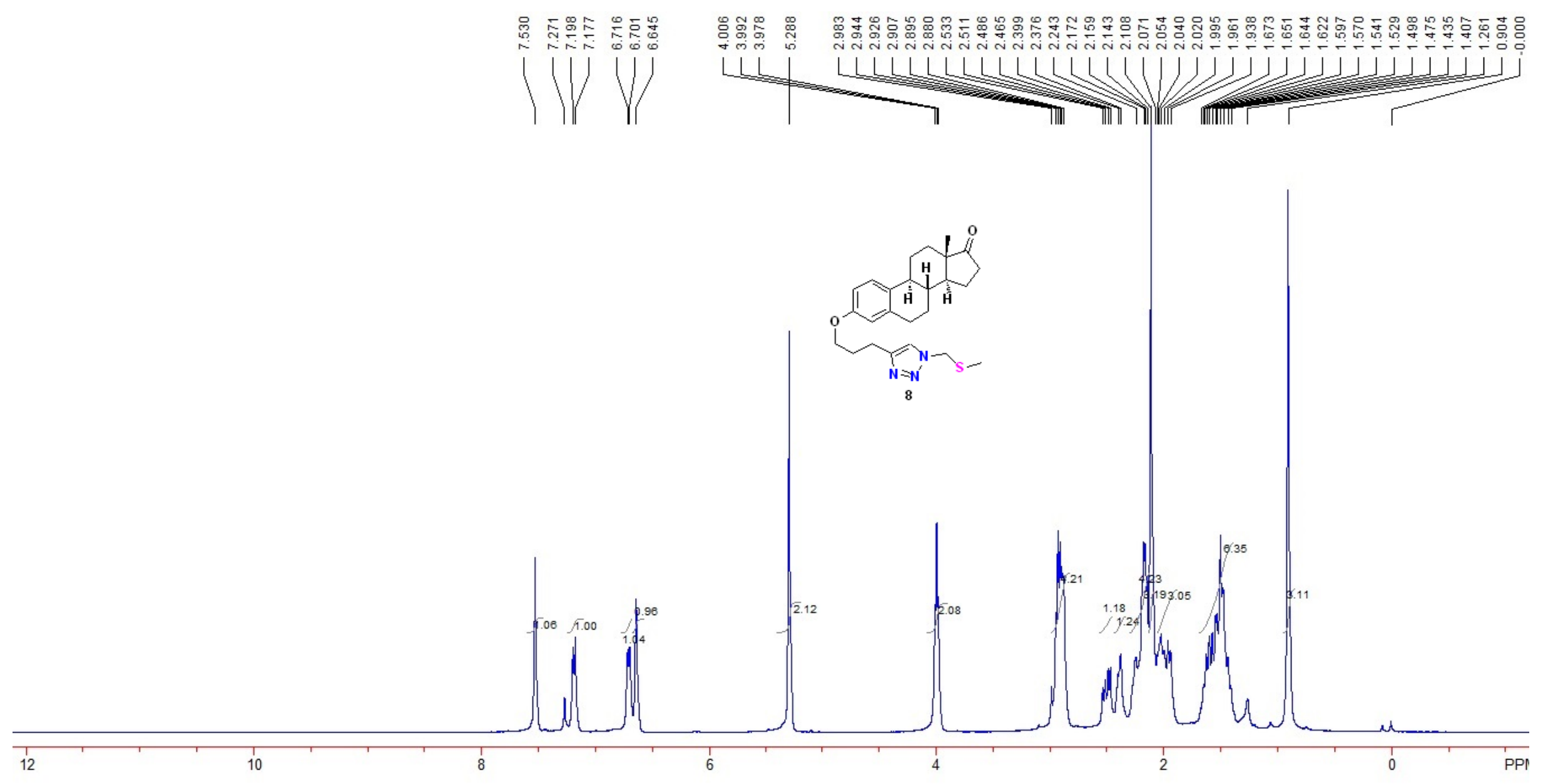



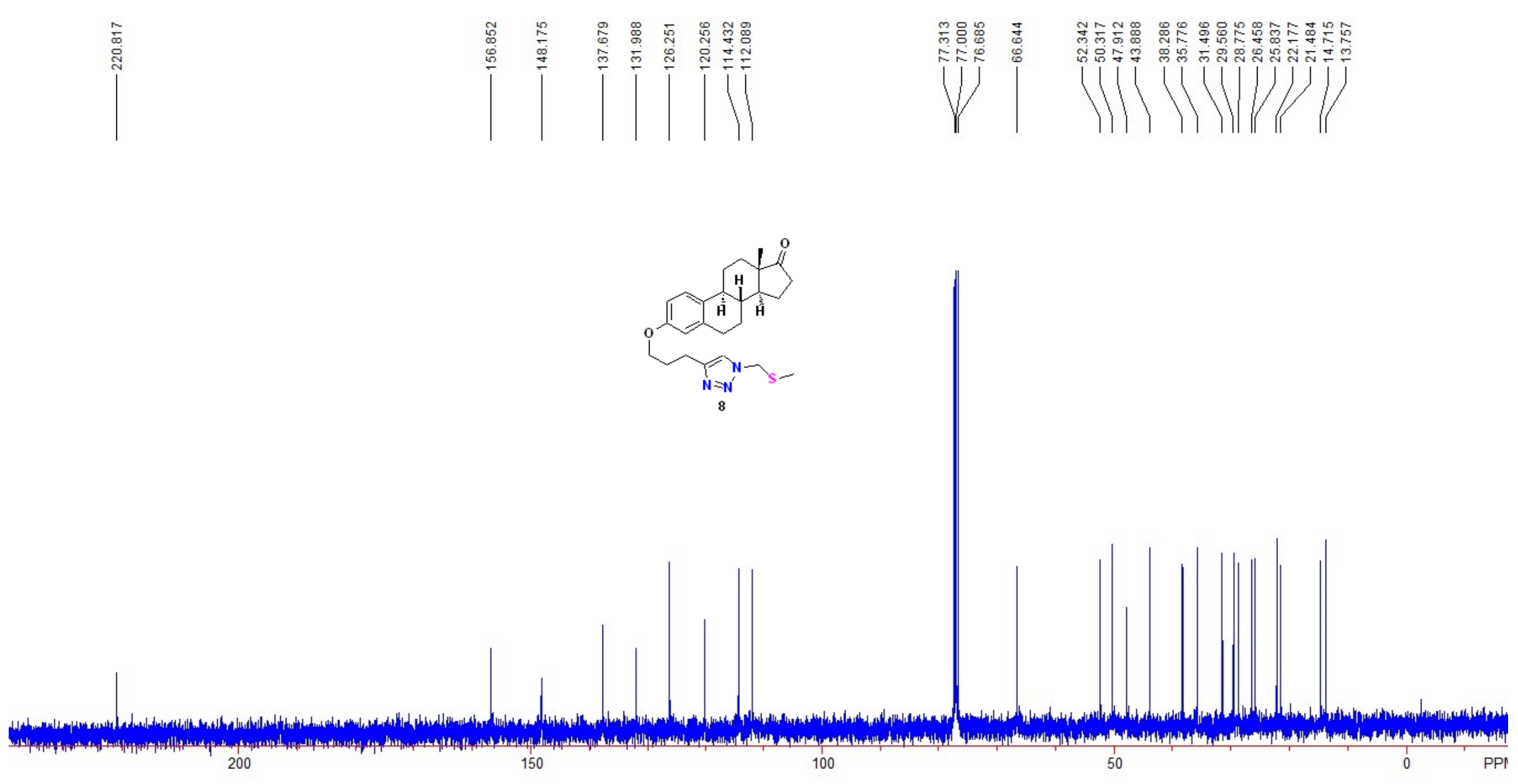


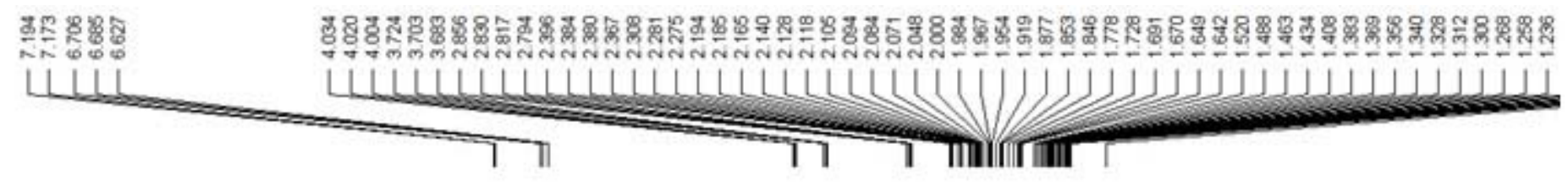

B77.78

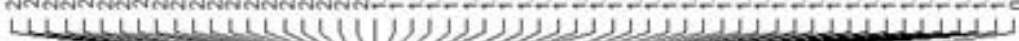

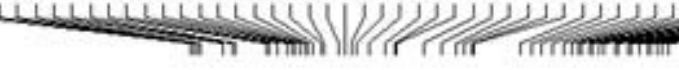
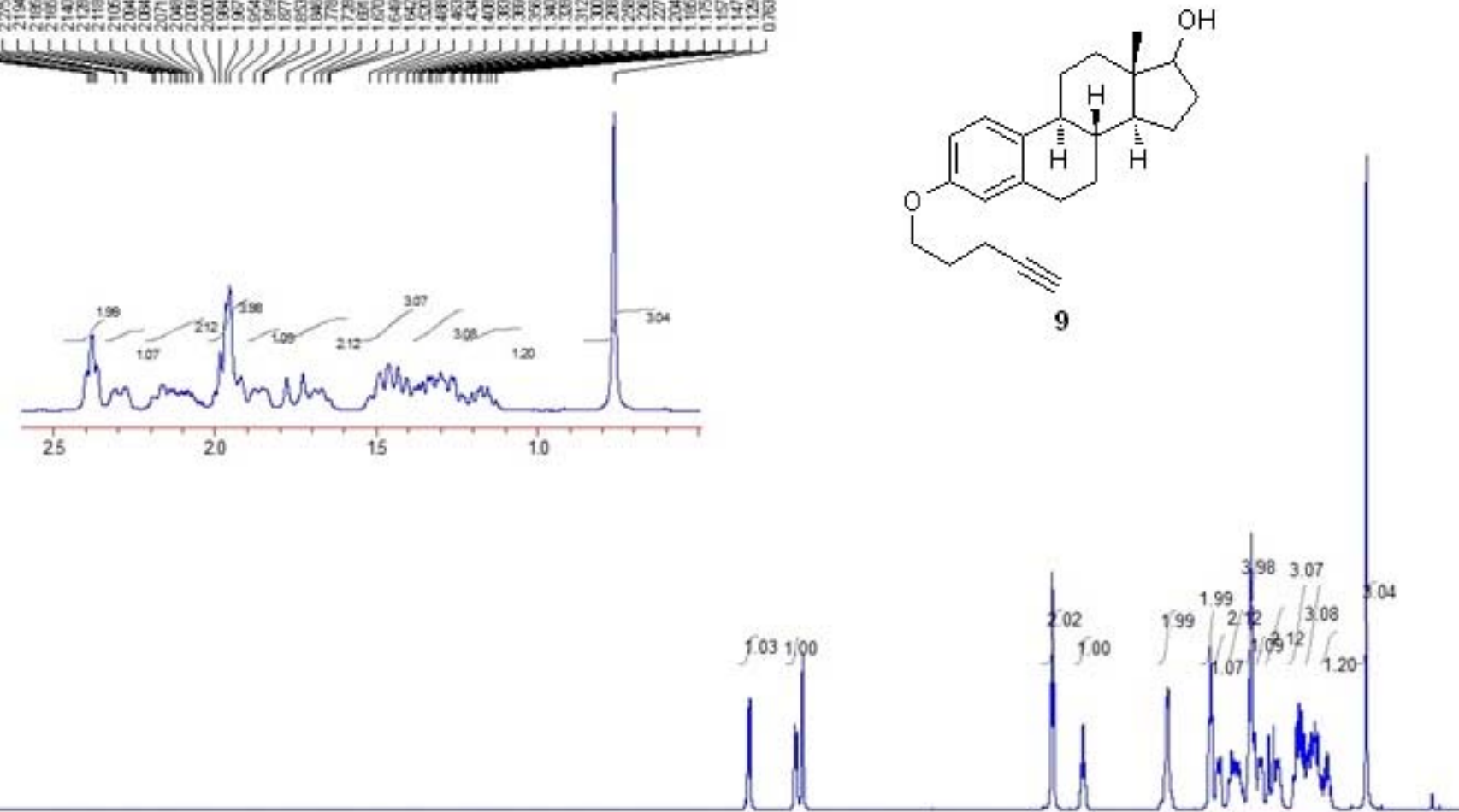

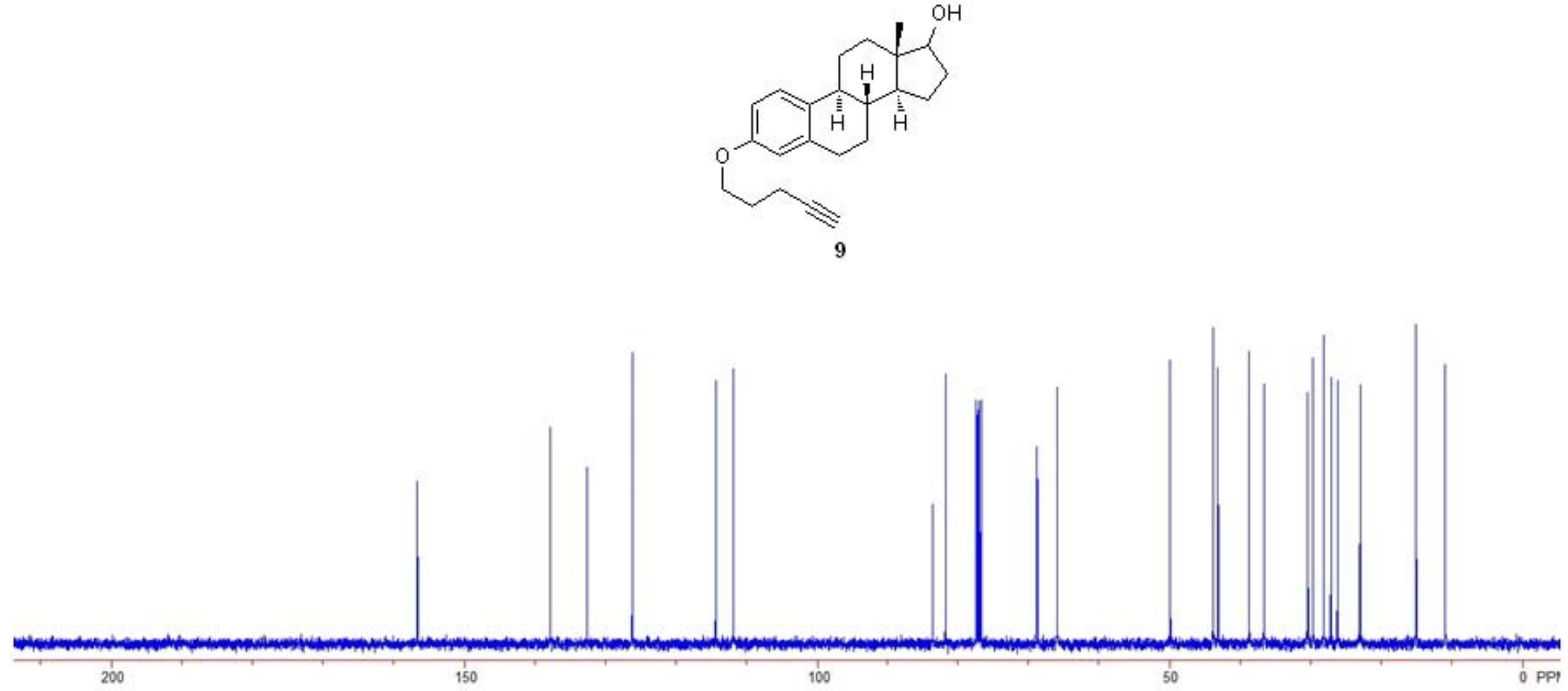

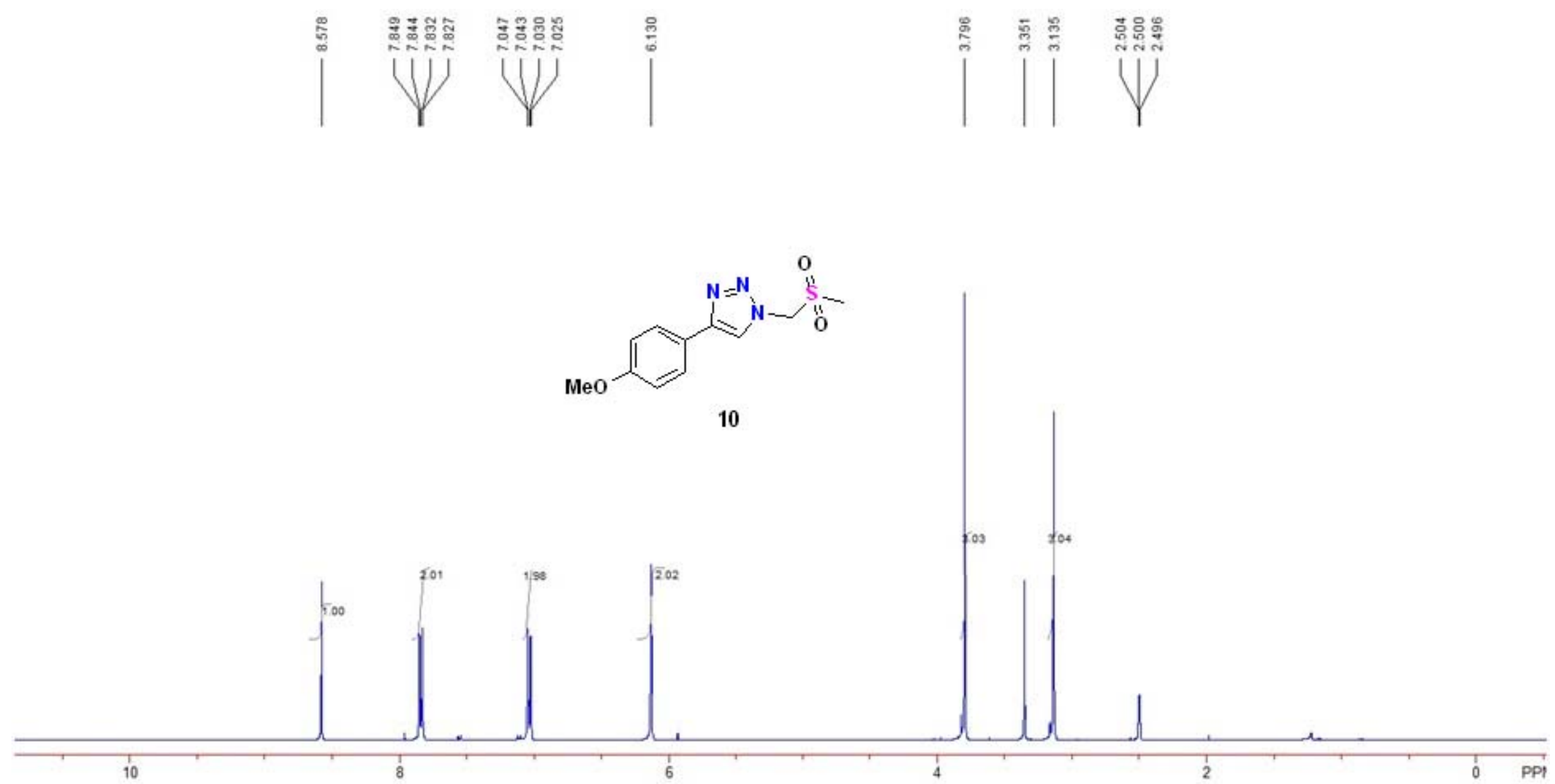

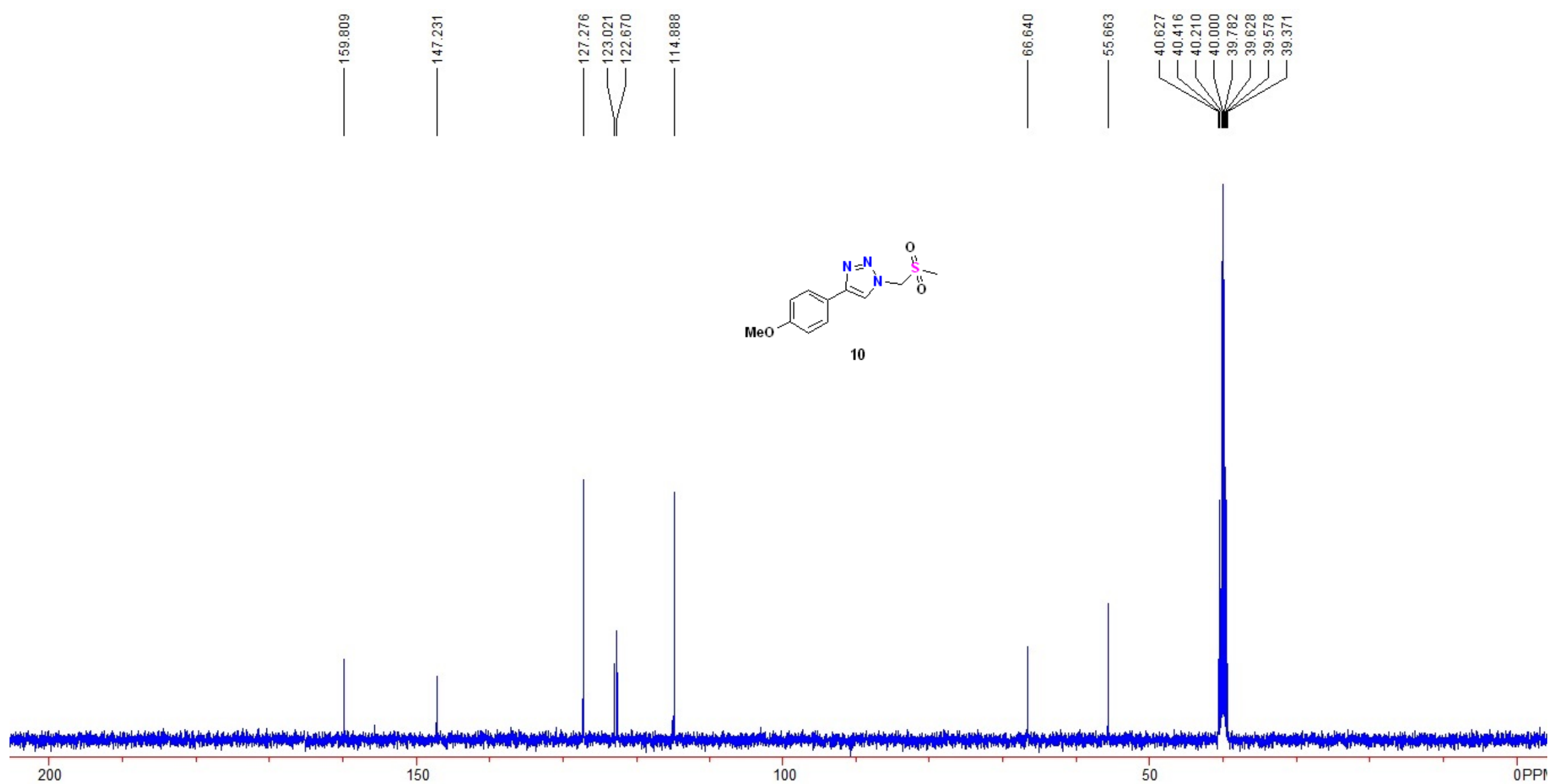


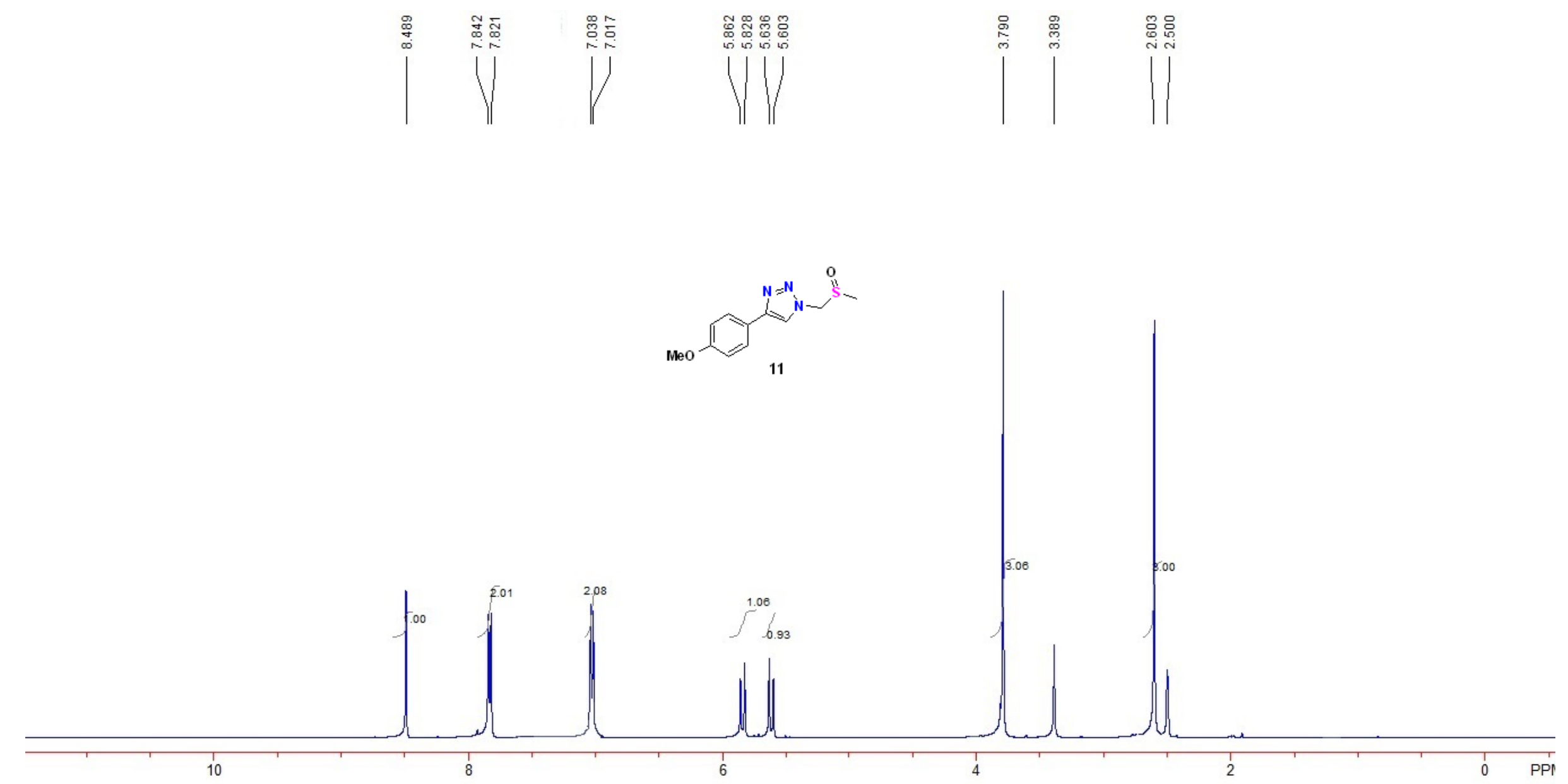



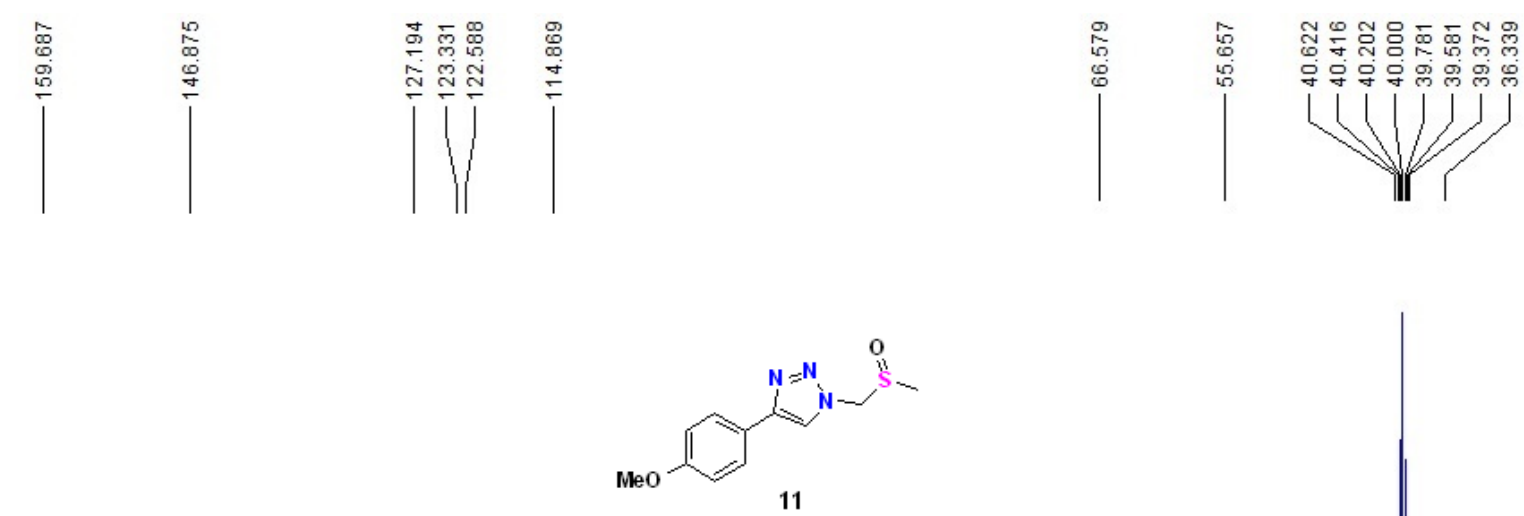

(100 


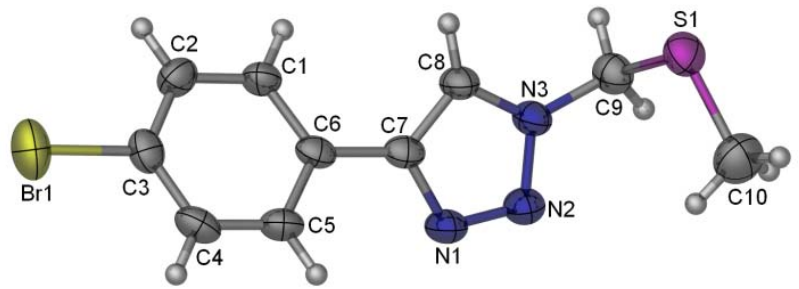

Fig. SI 1.X-ray Crystallography Structure of Compound 2e 